Bangladesh J. Plant Taxon. 27(1): 37-65, 2020 (June)

(C) 2020 Bangladesh Association of Plant Taxonomists

\title{
PRELIMINARY TAXONOMIC STUDY ON HOMESTEAD FLORA OF FOUR DISTRICTS OF BANGLADESH: MAGNOLIOPSIDA
}

\author{
Goutam Kumer Roy* and Saleh Ahammad Khan
}

Department of Botany, Jahangirnagar University, Savar, Dhaka-1342, Bangladesh

Keywords: Homestead flora; Magnoliopsida; Threatened Species; Four Districts; Bangladesh.

\begin{abstract}
This study has documented the contemporary taxonomic information on the species of the class Magnoliopsida (Dicotyledons) extant in the homestead areas of Dhaka, Gazipur, Manikganj and Tangail districts of Bangladesh. In these areas, the Dicotyledons are comprised of total 455 species under 302 genera belonging to 78 families. Fabaceae with 41 species is the largest family and Solanum and Lindernia are the largest genera. Total 238 species are herbs followed by 129 species of trees and 88 species of shrubs. Total 332 species are economically useful. The composition and distribution of the species of this plant group are remarkably variable in the homestead areas of the four districts. The current status of seven threatened species viz., Abroma augusta, Andrographis paniculata, Aniseia martinicensis, Mucuna bracteata, Pterocarpus santalinus, Rauvolfia serpentina and Tournefortia roxburghii, included in the Red Data Book of Bangladesh and extant in the study area, has been evaluated and described. This study has identified some threats to the homestead flora and formulated some recommendations for the conservation of threatened and declining native plant species of the study area. The data provided by this study will serve as an important baseline to track the trend of changes in the floristic composition and diversity and sustainable development of plant genetic resources in the homesteads of the study area.
\end{abstract}

\section{Introduction}

Dicotyledons are the most successful and dominant plant group (Heywood, 1993). They comprise about $62.5 \%$ of the total angiosperm species (ca. 400000) recorded from this planet so far (Leitch and Leitch, 2008). Dicots are the major biotic component of almost all ecosystems and essential for human being as the source of food, cloths, shelter, medicines, fodder and many other substances used in different puroses worldwide. Bangladesh is endowed with about 5,000 species of flowering plants, of which more than two third are Dicotyledonous (Khan, 1972-1987). Dicot plants dominate the forests, village groves and woodlands of Bangladesh (Khan and Afza, 1968; Khan and Banu, 1969; Khan, 1972-1987).

Homesteads are privately owned dwellings in the rural and semi-urban settings that typically consist of a living area, kitchen, backyard, front yard, and sometimes a pond and patio (Muhammed et al., 2011). A homestead forest, or "homegarden", is usually a complex mixture of deliberately planted vegetation and designed to produce natural products for the household uses or market sale. It is an operational unit, in which a number of crops are grown with livestock, poultry and fish production mainly for the purpose of the farmer's basic needs (Leuschner and Khaleque, 1987). Bangladesh has 15.4 million homesteads occupying 0.3 million hectares of land that are providing major requirement of food, fruit, vegetables, timber and fuel wood (Abedin and Quddus, 1990). Plants of the homesteads are receiving increasing attention from scientists, practitioners and policymakers because of their social, economic, ecological and environmental benefits (Alam, 2011).

*Author for correspondence, email: roy_kbd@yahoo.com 
In Bangladesh, there is no specific management plan for the homestead forests (FAO, 2010) and the household owners are traditionally managing these. It is estimated that about $70 \%$ of timber, $90 \%$ of firewood, $48 \%$ of sawn and veneer logs, and almost $90 \%$ of bamboo requirements are met from homestead forests (Uddin et, al., 2002). During the last 40-50 years, the relative importance has shifted from the traditional forestry to homestead plantation (Roy et al., 2013).

Previousely some detail floristic studies (Hooker, 1872-1897; Prain, 1903; Khan, 1972-1987; Ahmed et al., 2008-2009) were carried on covering the political boundary of Bangladesh. Many taxonomic inventories (Khan et al., 1977; Khan et al., 1985; Huq, 1986, 1988; Alam et al., 2006; Rahman et al., 2012; Rahman, 2013; Rahman et al., 2013; Rahaman et al., 2015; Shetu et al. 2018; Tabassum, 2015) were also performed in different urban and rural areas of this country. However, these studies do not provide specific data on the homestead forests or floras of the study area.

A good of number studies (Bashar, 1999; Uddin et al., 2002; Alam et al. 2005; Masum et al., 2008; Kabir and Webb, 2009; Miah and Hussain, 2010; Alam, 2011; Muhammed et al., 2011; Muhammed et al., 2013; Islam et al., 2013; Rahman et al., 2013; Roy et al., 2013; Islam et al., 2015; Rahaman et al., 2015; Sajib et. al., 2016) were carried out on the homestead forests in different regions of Bangladesh. However, the homestead floras of Dhaka, Manikganj, Gazipur and Tangail regions are not yet studied. This study was carried out to provide basic and recent taxonomic information on the species of the class Magnoliopsida (Dicotyledons) extant in the visited homestead areas of Bangladesh.

\section{Materials and Methods}

The study area lies in Dhaka, Gazipur, Manikganj and Tangail districts, that are located in central Bangladesh, in between $23^{\circ} 38^{\prime}-24^{\circ} 48^{\prime} \mathrm{N}$ and $89^{\circ} 41^{\prime}-90^{\circ} 42^{\prime} \mathrm{E}$ and composed of an area of about $8067.97 \mathrm{sq} \mathrm{km}$. This study was conducted during 2013 to 2018 based on a thorough taxonomic inventory involving 240 field trips to 1120 representative homestead areas belonging to 40 villages of 20 upazilas under the four districts in different seasons (Fig. 1).

Following a preliminary reconnaissance survey, four categories of homesteads viz., old joint, old isolated, and new joint and new isolated, were selected from each district for better representation. The selected old homesteads were more than 20 years to 80 years old and the new ones were more than five years to less than 20 years old. The homesteads of each district were equal in number, more or less homogenous and from plain land areas to promote the reasonable comparision. Representative specimens with flowers and fruits were collected during the field trips. Routine herbarium methods (Jain and Rao, 1977) have been followed in collecting, preparing, pressing, drying, mounting, and storing the plant specimens.

All plant specimens were identified through consulting with the experts, taxonomic descriptions and keys available in the relevant literature (Hooker, 1872-1897; Wu et al., 1995-2013), and matching with relevant voucher specimens preserved at Jahangirnagar University Herbarium (JUH), and Bangladesh National Herbarium (DACB). Nomenclatural information were verified through consulting TROPICOS (2010), The Plant List (2013), International Plant Names Index (2015) and the recent floras (Wu et al., 1995-2013; Watson et al., 2011). The families have been arranged following Cronquist (1981). The families Phyllanthaceae and Putranjivaceae, not included in Cronquist (1981)'s System, are placed besides their close family Euphorbiaceae. All genera and species are presented alphabetically. All voucher specimens are housed at JUH. The similarities in species composition in the homesteads of four districts have been measured following Jaccard coefficient (Jaccard, 1912). The threatened status

of the species in the homsteads of the study area was recognized based on their existing population 
size, distribution range, and regeneration in the area, collections, interviews with elderly local people, and consulting the relevent IUCN guidelines (IUCN Standards and Petitions Committee, 2019).

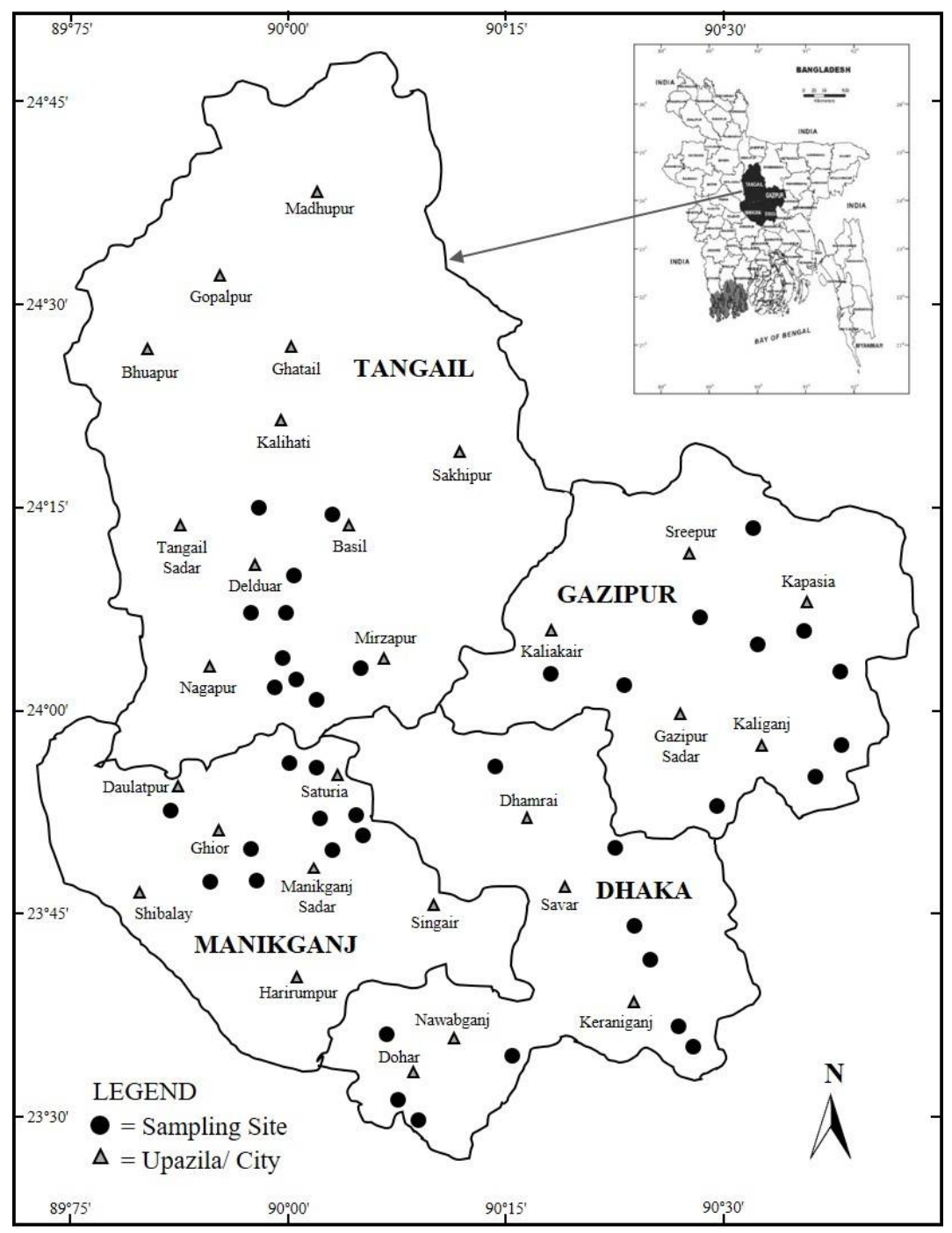

Fig. 1. Map showing the location of the the study area (Tangail, Gazipur, Manikganj and Dhaka districts) and sampling sites.

\section{Results and Discussion}

This study recorded total 455 species of Magnoliopsida (Dicotyledons) under 302 genera and 78 families from the the visited homestead areas of Dhaka, Gazipur, Manikganj and Tangail districts (Table 1). Twenty three of the families were represented by single species each, 39 families by 2-10 species and only 13 families by 11-20 species. The homesteads of four districts 
Table 1. Taxonomic checklist of the species of Magnoliopsida extant in the homestead areas of Dhaka, Gazipur, Manikganj and Tangail districts.

\begin{tabular}{|c|c|c|c|c|c|c|c|c|}
\hline Scientific Name & Bangla name & District & Habit & Habitat & Occ. & Fl./Fr. Time & Use & RSE \\
\hline ANNONACEAE & & & & & & & & \\
\hline Annona reticulata $\mathrm{L}$. & Nona & $\begin{array}{l}\mathrm{D}, \mathrm{G}, \\
\mathrm{M}, \mathrm{T}\end{array}$ & $\mathrm{T}$ & $\mathrm{Ah}, \mathrm{Fp}$ & $\mathrm{C}$ & $\begin{array}{l}\text { Fl. May-Jul } \\
\text { Fr. Sep-Jan }\end{array}$ & Fr & GKR2262 \\
\hline A. squamosa $\mathrm{L}$. & Ata & $\begin{array}{l}\mathrm{D}, \mathrm{G}, \\
\mathrm{M}, \mathrm{T}\end{array}$ & $\mathrm{T}$ & $\mathrm{Ah}$ & $\mathrm{C}$ & $\begin{array}{l}\text { Fl. Apr-May } \\
\text { Fr. Aug-Nov }\end{array}$ & Fr & GKR0671 \\
\hline $\begin{array}{l}\text { Miliusa velutina (A.DC.) } \\
\text { Hook.f. \& Thomson }\end{array}$ & Gandhigajari & $\mathrm{G}, \mathrm{M}$ & $\mathrm{T}$ & $\mathrm{Ah}, \mathrm{Fp}$ & $\mathrm{O}$ & $\begin{array}{l}\text { Fl. Dec-May } \\
\text { Fr. U }\end{array}$ & $\mathrm{Fw}$ & GKR1679 \\
\hline $\begin{array}{l}\text { Polyalthia longifolia (Sonn.) } \\
\text { Thwaites }\end{array}$ & Debdaru & $\begin{array}{l}\mathrm{D}, \mathrm{G}, \\
\mathrm{M}, \mathrm{T}\end{array}$ & $\mathrm{T}$ & $\mathrm{Fp}, \mathrm{Rs}$ & $\mathrm{C}$ & $\begin{array}{l}\text { Fl. Mar-May } \\
\text { Fr. Jul-Sep }\end{array}$ & $\mathrm{T}$ & GKR0697 \\
\hline $\begin{array}{l}\text { P. suberosa (Roxb.) } \\
\text { Thwaites }\end{array}$ & $\begin{array}{l}\text { Hamjam; } \\
\text { Murmuri }\end{array}$ & $\begin{array}{l}\mathrm{D}, \mathrm{G}, \\
\mathrm{M}, \mathrm{T}\end{array}$ & $\mathrm{S}$ & $\mathrm{Fp}, \mathrm{Rs}$ & $\mathrm{C}$ & $\begin{array}{l}\text { Fl. Sep-Mar } \\
\text { Fr. U }\end{array}$ & W & GKR1106 \\
\hline Uvaria littoralis Blume & Gagh-ranga & G & $\mathrm{S}$ & $\mathrm{Fp}$ & $\mathrm{O}$ & $\begin{array}{l}\text { Fl. Jul-Aug } \\
\text { Fr. U }\end{array}$ & $\mathrm{W}$ & GKR1133 \\
\hline $\begin{array}{l}\text { LAURACEAE } \\
\text { Cinnamomum tamala } \\
\text { (Buch.-Ham.) T. Nees \& } \\
\text { Eberm. }\end{array}$ & Tejpata & $\mathrm{D}, \mathrm{M}$ & $\mathrm{T}$ & $\mathrm{Ah}$ & $\mathrm{O}$ & $\begin{array}{l}\text { Fl. Feb-Oct } \\
\text { Fr. U }\end{array}$ & $\mathrm{S}$ & GKR0172 \\
\hline C. zeylanicum Blume & Daruchini & $\mathrm{M}, \mathrm{T}$ & ST & $\mathrm{Ah}$ & $\mathrm{O}$ & $\begin{array}{l}\text { Fl. Apr-Jun } \\
\text { Fr. U }\end{array}$ & $\mathrm{S}$ & GKR1572 \\
\hline $\begin{array}{l}\text { Litsea glutinosa (Lour.) } \\
\text { C.B.Rob. }\end{array}$ & Kukurchita & $\mathrm{D}, \mathrm{T}$ & $\mathrm{T}$ & Ah, Rs & $\mathrm{O}$ & $\begin{array}{l}\text { Fl. Apr-May } \\
\text { Fr. U }\end{array}$ & $\mathrm{W}$ & GKR2895 \\
\hline $\begin{array}{l}\text { L. salicifolia (J. Roxb. ex } \\
\text { Nees) Hook. f. }\end{array}$ & $\begin{array}{l}\text { Bara shiyal- } \\
\text { buka, Digloti }\end{array}$ & $\mathrm{D}, \mathrm{M}$ & $\mathrm{T}$ & Rs & $\mathrm{O}$ & $\begin{array}{l}\text { Fl. Apr-May } \\
\text { Fr. U }\end{array}$ & $\mathrm{W}$ & GKR0145 \\
\hline PIPERACEAE & & & & & & & & \\
\hline $\begin{array}{l}\text { Peperomia pellucida (L.) } \\
\text { Kunth }\end{array}$ & Luchipata & $\begin{array}{l}\mathrm{D}, \mathrm{G} \\
\mathrm{M}, \mathrm{T}\end{array}$ & $\mathrm{H}$ & $\begin{array}{l}\text { Ah, Fp, } \\
\text { Rs }\end{array}$ & $\mathrm{C}$ & $\begin{array}{l}\text { Fl. Sep-Dec } \\
\text { Fr. U }\end{array}$ & We & GKR0512 \\
\hline Piper betle L. & Pan & $\mathrm{M}, \mathrm{T}$ & $\mathrm{H}$ & $\mathrm{Ah}$ & $\mathrm{O}$ & $\begin{array}{l}\text { Fl. Apr-May } \\
\text { Fr. U }\end{array}$ & M & GKR1571 \\
\hline P. longum $\mathrm{L}$. & Pipul & $\mathrm{D}, \mathrm{T}$ & $\mathrm{H}$ & $\mathrm{Ah}, \mathrm{Fp}$ & $\mathrm{O}$ & $\begin{array}{l}\text { Fl. Aug-Jan } \\
\text { Fr. U }\end{array}$ & M & GKR0045 \\
\hline P. peepuloides Roxb. & wild pepper & $\mathrm{M}, \mathrm{T}$ & $\mathrm{H}$ & $\begin{array}{l}\text { Ah, Fp, } \\
\text { Rs }\end{array}$ & $\mathrm{O}$ & $\begin{array}{l}\text { Fl. Nov-Dec } \\
\text { Fr. U }\end{array}$ & W & GKR1125 \\
\hline P. peploides (Kunth) Poir. & Pipul & $\mathrm{D}, \mathrm{M}$ & $\mathrm{T}$ & $\mathrm{Ah}$ & $\mathrm{O}$ & $\begin{array}{l}\text { Fl. Jun-Aug } \\
\text { Fr. Nov-Dec }\end{array}$ & W & GKR1272 \\
\hline P. sylvaticum Roxb. & Pahari pipol & $\mathrm{D}$ & $\mathrm{H}$ & Rs & $\mathrm{O}$ & $\begin{array}{l}\text { Fl. Aug-Sep } \\
\text { Fr. U }\end{array}$ & $\mathrm{W}$ & GKR1544 \\
\hline $\begin{array}{l}\text { ARISTOLOCHIACEAE } \\
\text { Aristolochia indica L. }\end{array}$ & Ishwarmul & $\begin{array}{l}\mathrm{G}, \mathrm{M}, \\
\mathrm{T}\end{array}$ & $\mathrm{H}$ & $\begin{array}{l}\text { Ah, Fp, } \\
\text { Rs }\end{array}$ & $\mathrm{O}$ & $\begin{array}{l}\text { Fl. Jul-Mar } \\
\text { Fr. U }\end{array}$ & M & GKR0038 \\
\hline $\begin{array}{l}\text { RANUNCULACEAE } \\
\text { Ranunculus sceleratus L. }\end{array}$ & Palik, Podika & M & $\mathrm{H}$ & Fp & $\mathrm{O}$ & $\begin{array}{l}\text { Fl. Feb-May } \\
\text { Fr. U }\end{array}$ & M & GKR0062 \\
\hline $\begin{array}{l}\text { MENISPERMACEAE } \\
\text { Cocculus hirsutus (L.) W. } \\
\text { Theob. }\end{array}$ & $\begin{array}{l}\text { Doipata, } \\
\text { Jhaljamani }\end{array}$ & G & $\mathrm{H}$ & Fp & $\mathrm{O}$ & $\begin{array}{l}\text { Fl. \& Fr. Through- } \\
\text { out the year }\end{array}$ & M & GKR0334 \\
\hline $\begin{array}{l}\text { Stephania japonica (Thunb.) } \\
\text { Miers }\end{array}$ & $\begin{array}{l}\text { Nimukha, } \\
\text { Aknadi }\end{array}$ & $\mathrm{D}, \mathrm{M}$ & $\mathrm{S}$ & $\begin{array}{l}\text { Ah, Fp, } \\
\text { Rs }\end{array}$ & $\mathrm{O}$ & $\begin{array}{l}\text { Fl. Mar-Sep } \\
\text { Fr. U }\end{array}$ & M & GKR0095 \\
\hline
\end{tabular}

(Table contd.) 


\begin{tabular}{|c|c|c|c|c|c|c|c|c|}
\hline Scientific Name & Bangla name & District & Habit & Habitat & Occ. & Fl./Fr. Time & Use & RSE \\
\hline Tiliacora acuminata Miers & Baghlata & $\begin{array}{l}\mathrm{G}, \mathrm{M}, \\
\mathrm{T}\end{array}$ & $\mathrm{S}$ & $\begin{array}{l}\text { Ah, Fp, } \\
\text { Rs }\end{array}$ & $\mathrm{O}$ & $\begin{array}{l}\text { Fl. Apr-Dec } \\
\text { Fr. U }\end{array}$ & $\mathrm{M}$ & GKR0122 \\
\hline $\begin{array}{l}\text { Tinospora crispa (L.) Hook. } \\
\text { f. \& Thomson }\end{array}$ & Gulancha & $\mathrm{D}$ & $\mathrm{S}$ & Rs & $\mathrm{O}$ & $\begin{array}{l}\text { Fl. Feb-Jun } \\
\text { Fr. U }\end{array}$ & M & GKR1344 \\
\hline T. sinensis (Lour.) Merr. & $\begin{array}{l}\text { China } \\
\text { gulancha }\end{array}$ & $\mathrm{G}, \mathrm{T}$ & $\mathrm{S}$ & $\mathrm{Ah}, \mathrm{Fp}$ & $\mathrm{O}$ & $\begin{array}{l}\text { Fl. Feb-Jun } \\
\text { Fr. U }\end{array}$ & M & GKR0304 \\
\hline \multicolumn{9}{|l|}{ ULMACEAE } \\
\hline $\begin{array}{l}\text { Holoptelea integrifolia } \\
\text { Planch. }\end{array}$ & nata karanja & G & $\mathrm{T}$ & $\mathrm{Ah}, \mathrm{Fp}$ & $\mathrm{O}$ & $\begin{array}{l}\text { Fl. \& Fr. Dec- } \\
\text { Mar }\end{array}$ & Fw & GKR0359 \\
\hline \multicolumn{9}{|l|}{ CANNABACEAE } \\
\hline Cannabis sativa $\mathrm{L}$. & Gaza & $\mathrm{M}, \mathrm{T}$ & $\mathrm{H}$ & Fp, Rs & $\mathrm{O}$ & $\begin{array}{l}\text { Fl. Aug-Mar } \\
\text { Fr. U }\end{array}$ & M & GKR0139 \\
\hline Trema orientalis (L.) Blume & Jibon & $\begin{array}{l}\mathrm{D}, \mathrm{G} \\
\mathrm{M}, \mathrm{T}\end{array}$ & $\mathrm{T}$ & Fp, Rs & $\mathrm{C}$ & $\begin{array}{l}\text { Fl. Sep-Dec } \\
\text { Fr. U }\end{array}$ & $\begin{array}{l}\text { M, } \\
\text { Fw }\end{array}$ & GKR0570 \\
\hline \multicolumn{9}{|l|}{ MORACEAE } \\
\hline $\begin{array}{l}\text { Artocarpus heterophyllus } \\
\text { Lam. }\end{array}$ & Kanthal & $\begin{array}{l}\mathrm{D}, \mathrm{G}, \\
\mathrm{M}\end{array}$ & $\mathrm{T}$ & Ah, Rs & $\mathrm{O}$ & $\begin{array}{l}\text { Fl. Jan-Jun } \\
\text { Fr. U }\end{array}$ & $\begin{array}{l}\text { Fr, } \\
\mathrm{T}\end{array}$ & GKR 0174 \\
\hline A. lacucha Buch.-Ham. & Dewa & $\begin{array}{l}\mathrm{D}, \mathrm{G}, \\
\mathrm{M}, \mathrm{T}\end{array}$ & $\mathrm{T}$ & $\begin{array}{l}\text { Ah, Fp, } \\
\text { Rs }\end{array}$ & $\mathrm{C}$ & $\begin{array}{l}\text { Fl. Feb-May } \\
\text { Fr. U }\end{array}$ & $\begin{array}{l}\text { Fr, } \\
\text { Fw }\end{array}$ & GKR2082 \\
\hline Ficus benghalensis $\mathrm{L}$. & Bot & $\begin{array}{l}\mathrm{D}, \mathrm{G}, \\
\mathrm{M}, \mathrm{T}\end{array}$ & $\mathrm{T}$ & $\mathrm{Fp}$ & $\mathrm{C}$ & $\begin{array}{l}\text { Fl. May-Jul } \\
\text { Fr. U }\end{array}$ & $\begin{array}{l}\text { M, } \\
\text { Fw }\end{array}$ & GKR0310 \\
\hline F. carica $\mathrm{L}$. & Dumur, Anjir & $\begin{array}{l}\mathrm{D}, \mathrm{G}, \\
\mathrm{M}, \mathrm{T}\end{array}$ & $\mathrm{T}$ & $\mathrm{Ah}, \mathrm{Fp}$ & $\mathrm{C}$ & $\begin{array}{l}\text { Fl. May-Aug } \\
\text { Fr. U }\end{array}$ & M & GKR0079 \\
\hline F. heterophylla L. f. & Bhuidumur & $\begin{array}{l}\mathrm{D}, \mathrm{G}, \\
\mathrm{M}, \mathrm{T}\end{array}$ & $\mathrm{S}$ & $\begin{array}{l}\text { Ah, Fp, } \\
\text { Rs }\end{array}$ & $\mathrm{C}$ & $\begin{array}{l}\text { Fl. Nov-Jan } \\
\text { Fr. U }\end{array}$ & $\mathrm{Fw}$ & GKR0033 \\
\hline F. hispida L. f. & Kakdumur & $\begin{array}{l}\mathrm{D}, \mathrm{G}, \\
\mathrm{M}, \mathrm{T}\end{array}$ & $\mathrm{T}$ & $\begin{array}{l}\text { Ah, Fp, } \\
\text { Rs }\end{array}$ & $\mathrm{C}$ & Fl. Sep-May & $\begin{array}{l}\text { Fr, } \\
\text { Fw }\end{array}$ & GKR0577 \\
\hline F. racemosa $\mathrm{L}$. & Jogo dumur & $\begin{array}{l}\mathrm{D}, \mathrm{G} \\
\mathrm{M}, \mathrm{T}\end{array}$ & $\mathrm{T}$ & $\begin{array}{l}\text { Ah, Fp, } \\
\text { Rs }\end{array}$ & $\mathrm{C}$ & $\begin{array}{l}\text { Fr. U } \\
\text { Fl. Feb-May } \\
\text { Fr. U }\end{array}$ & $\begin{array}{l}\text { Fr, } \\
\text { Fw, } \\
\text { M }\end{array}$ & GKR1763 \\
\hline F. religiosa $\mathrm{L}$. & Ashatha & $\mathrm{D}, \mathrm{G}$ & $\mathrm{T}$ & $\mathrm{Ah}, \mathrm{Fp}$ & $\mathrm{O}$ & Fl. \& Fr. Nov-Feb & M & GKR3132 \\
\hline F. rumphii Blume & Pakur & $\mathrm{D}, \mathrm{M}$ & $\mathrm{T}$ & $\mathrm{Ah}, \mathrm{Rs}$ & $\mathrm{O}$ & $\begin{array}{l}\text { Fl. Mar-Nov } \\
\text { Fr. U }\end{array}$ & Fw & GKR1324 \\
\hline Morus alba $\mathrm{L}$. & Tut & M & ST & Rs & $\mathrm{O}$ & $\begin{array}{l}\text { Fl. Apr-Aug } \\
\text { Fr. U }\end{array}$ & M & GKR1243 \\
\hline $\begin{array}{l}\text { Premna bengalensis } \\
\text { C.B.Clarke }\end{array}$ & Koya-jarul & $\mathrm{D}$ & $\mathrm{T}$ & Fp & $\mathrm{O}$ & $\begin{array}{l}\text { Fl. May-Nov } \\
\text { Fr. U }\end{array}$ & $\mathrm{W}$ & GKR1607 \\
\hline Streblus asper Lour. & Sheora & $\begin{array}{l}\mathrm{D}, \mathrm{G} \\
\mathrm{M}\end{array}$ & $\mathrm{H}$ & $\begin{array}{l}\mathrm{Bb}, \mathrm{Ep}, \\
\mathrm{Fp}\end{array}$ & $\mathrm{O}$ & Fl. \& Fr. Feb-Jun & M & GKR1822 \\
\hline \multicolumn{9}{|l|}{ URTICACEAE } \\
\hline $\begin{array}{l}\text { Gonostegia hirta (Blume ex } \\
\text { Hassk.)Miq. }\end{array}$ & Unknown & $\mathrm{G}, \mathrm{M}$ & ST & $\begin{array}{l}\text { Ah, Fp, } \\
\text { Rs }\end{array}$ & $\mathrm{O}$ & $\begin{array}{l}\text { Fl. Jun-Jul } \\
\text { Fr. Aug-Sep }\end{array}$ & $\begin{array}{l}\mathrm{M} \\
\mathrm{Fd}\end{array}$ & GKR0175 \\
\hline $\begin{array}{l}\text { Laportea interrupta (L.) } \\
\text { Chew }\end{array}$ & bichuti & $\begin{array}{l}\mathrm{D}, \mathrm{G} \\
\mathrm{M}, \mathrm{T}\end{array}$ & $\mathrm{H}$ & $\begin{array}{l}\text { Ah, Fp, } \\
\text { Rs }\end{array}$ & $\mathrm{C}$ & $\begin{array}{l}\text { Fl. Jul-Sep } \\
\text { Fr. U }\end{array}$ & $\mathrm{W}$ & GKR0861 \\
\hline $\begin{array}{l}\text { Pilea microphylla (L.) } \\
\text { Liebm. }\end{array}$ & Rockweed & $\mathrm{T}$ & $\mathrm{H}$ & $\mathrm{Ah}$ & $\mathrm{O}$ & $\begin{array}{l}\text { Fl. Aug-Nov } \\
\text { Fr. U }\end{array}$ & $\mathrm{We}$ & GKR1065 \\
\hline $\begin{array}{l}\text { Pouzolzia zeylanica }(\mathrm{L} .) \\
\text { Benn. }\end{array}$ & $\begin{array}{l}\text { Bilati } \\
\text { luchipata }\end{array}$ & $\begin{array}{l}\mathrm{D}, \mathrm{G}, \\
\mathrm{M}, \mathrm{T}\end{array}$ & $\mathrm{H}$ & $\begin{array}{l}\text { Ah, Fp, } \\
\text { Rs }\end{array}$ & $\mathrm{C}$ & $\begin{array}{l}\text { Fl. Aug-Dec } \\
\text { Fr. U }\end{array}$ & $\mathrm{W}$ & GKR1072 \\
\hline
\end{tabular}




\begin{tabular}{|c|c|c|c|c|c|c|c|c|}
\hline Scientific Name & Bangla name & District & Habit & Habitat & Occ. & Fl./Fr. Time & Use & RSE \\
\hline \multicolumn{9}{|l|}{ NYCTAGINACEAE } \\
\hline Boerhavia diffusa $\mathrm{L}$. & Punarnova & $\mathrm{G}, \mathrm{T}$ & $\mathrm{H}$ & $\begin{array}{l}\text { Ah, Fp, } \\
\text { Rs }\end{array}$ & $\mathrm{O}$ & $\begin{array}{l}\text { Fl. Nov-Feb } \\
\text { Fr. U }\end{array}$ & M & GKR2156 \\
\hline Mirabilis jalapa $\mathrm{L}$. & Sandhyamaloti & $\begin{array}{l}\mathrm{G}, \mathrm{M}, \\
\mathrm{T}\end{array}$ & $\mathrm{H}$ & $\begin{array}{l}\text { Ah, Fp, } \\
\text { Rs }\end{array}$ & $\mathrm{O}$ & $\begin{array}{l}\text { Fl. Jun-Oct } \\
\text { Fr. U }\end{array}$ & Or & GKR0046 \\
\hline \multicolumn{9}{|l|}{ CHENOPODIACEAE } \\
\hline Chenopodium album $\mathrm{L}$. & Bathuashak & $\begin{array}{l}\mathrm{D}, \mathrm{G}, \\
\mathrm{M}, \mathrm{T}\end{array}$ & $\mathrm{H}$ & $\mathrm{Ah}, \mathrm{Fp}$ & $\mathrm{C}$ & $\begin{array}{l}\text { Fl. Feb-Mar } \\
\text { Fr. U }\end{array}$ & $\mathrm{V}$ & GKR0620 \\
\hline C. ambrosioides $\mathrm{L}$. & Chandanbeto & $\mathrm{D}, \mathrm{T}$ & $\mathrm{H}$ & $\mathrm{Ah}, \mathrm{Fp}$ & $\mathrm{O}$ & $\begin{array}{l}\text { Fl. \& Fr. Through- } \\
\text { out the year }\end{array}$ & $\mathrm{V}$ & GKR0553 \\
\hline \multicolumn{9}{|l|}{ AMARANTHACEAE } \\
\hline Achyranthes aspera $\mathrm{L}$. & $\begin{array}{l}\text { Apang, } \\
\text { Bilaiachra }\end{array}$ & $\begin{array}{l}\mathrm{D}, \mathrm{G}, \\
\mathrm{M}, \mathrm{T}\end{array}$ & $\mathrm{H}$ & $\begin{array}{l}\text { Ah, Fp, } \\
\text { Rs }\end{array}$ & $\mathrm{C}$ & $\begin{array}{l}\text { Fl. Jan-Aug } \\
\text { Fr. U }\end{array}$ & M & GKR0297 \\
\hline $\begin{array}{l}\text { Alternanthera } \\
\text { paronychioides A.St. Hil. }\end{array}$ & Jhuli khata & $\begin{array}{l}\mathrm{D}, \mathrm{G}, \\
\mathrm{M}, \mathrm{T}\end{array}$ & $\mathrm{H}$ & $\begin{array}{l}\text { Ah, Fp, } \\
\text { Rs }\end{array}$ & $\mathrm{C}$ & $\begin{array}{l}\text { Fl. Jun-Aug } \\
\text { Fr. U }\end{array}$ & $\mathrm{W}$ & GKR0051 \\
\hline $\begin{array}{l}\text { A. philoxeroides (Mart.) } \\
\text { Griseb. }\end{array}$ & $\begin{array}{l}\text { Haicha, } \\
\text { Malancha }\end{array}$ & $\begin{array}{l}\mathrm{D}, \mathrm{G}, \\
\mathrm{M}\end{array}$ & $\mathrm{H}$ & $\begin{array}{l}\text { Ah, Fp, } \\
\text { Rs }\end{array}$ & $\mathrm{O}$ & $\begin{array}{l}\text { Fl. Jan-Sep } \\
\text { Fr. U }\end{array}$ & $\mathrm{V}$ & GKR0083 \\
\hline A. sessilis (L.) R.Br. ex DC. & Chanchi & $\begin{array}{l}\mathrm{D}, \mathrm{G} \\
\mathrm{M}, \mathrm{T}\end{array}$ & $\mathrm{H}$ & $\begin{array}{l}\text { Ah, Fp, } \\
\text { Rs }\end{array}$ & $\mathrm{C}$ & $\begin{array}{l}\text { Fl.\& Fr. Through- } \\
\text { out the year }\end{array}$ & $\mathrm{V}$ & GKR0439 \\
\hline Amaranthus blitum $\mathrm{L}$. & Boutuni & $\begin{array}{l}\mathrm{D}, \mathrm{G}, \\
\mathrm{M}, \mathrm{T}\end{array}$ & $\mathrm{H}$ & $\mathrm{Ah}, \mathrm{Fp}$ & $\mathrm{C}$ & $\begin{array}{l}\text { Fl. Mar-Jun } \\
\text { Fr. U }\end{array}$ & $\mathrm{V}$ & GKR0174 \\
\hline A. gangeticus $\mathrm{L}$. & Data shak & $\mathrm{D}, \mathrm{M}$ & $\mathrm{H}$ & $\mathrm{Ah}, \mathrm{Fp}$ & $\mathrm{O}$ & $\begin{array}{l}\text { Fl. Jun-Sep } \\
\text { Fr. U }\end{array}$ & $\mathrm{V}$ & GKR0547 \\
\hline A. spinosus $\mathrm{L}$. & Katanotey & $\begin{array}{l}\mathrm{D}, \mathrm{G}, \\
\mathrm{M}, \mathrm{T}\end{array}$ & $\mathrm{H}$ & Fp, Rs & $\mathrm{C}$ & $\begin{array}{l}\text { Fl. Jun-Dec } \\
\text { Fr. U }\end{array}$ & $\mathrm{V}$ & GKR0461 \\
\hline A. viridis $\mathrm{L}$. & Notey shak & $\begin{array}{l}\mathrm{D}, \mathrm{G}, \\
\mathrm{M}, \mathrm{T}\end{array}$ & $\mathrm{H}$ & $\begin{array}{l}\text { Ah, Fp, } \\
\text { Rs }\end{array}$ & $\mathrm{C}$ & $\begin{array}{l}\text { Fl. Jul-Dec } \\
\text { Fr. U }\end{array}$ & $\mathrm{V}$ & GKR0507 \\
\hline Atriplex hortensis L. & $\begin{array}{l}\text { Red mountain } \\
\text { spinach }\end{array}$ & $\mathrm{D}$ & $\mathrm{H}$ & $\mathrm{Fp}$ & $\mathrm{O}$ & $\begin{array}{l}\text { Fl. Jul-Sep } \\
\text { Fr. U }\end{array}$ & $\begin{array}{l}\mathrm{M}, \\
\text { Or }\end{array}$ & GKR0656 \\
\hline Celosia argentea $\mathrm{L}$. & $\begin{array}{l}\text { Swetmorogph } \\
\text { ul }\end{array}$ & $\mathrm{D}$ & $\mathrm{H}$ & $\mathrm{Ah}, \mathrm{Fp}$ & $\mathrm{O}$ & $\begin{array}{l}\text { Fl. Apr-May } \\
\text { Fr. U }\end{array}$ & Or & GKR0568 \\
\hline $\begin{array}{l}\text { Cyathula prostrata }(\mathrm{L} .) \\
\text { Blume }\end{array}$ & Chhoto Apang & $\begin{array}{l}\mathrm{D}, \mathrm{G}, \\
\mathrm{M}, \mathrm{T}\end{array}$ & $\mathrm{H}$ & $\begin{array}{l}\text { Ah, Fp, } \\
\text { Rs }\end{array}$ & $\mathrm{C}$ & $\begin{array}{l}\text { Fl. Sep-Apr } \\
\text { Fr. U }\end{array}$ & M & GKR0166 \\
\hline Enhydra fluctuans DC & Helencha & $\mathrm{D}$ & $\mathrm{H}$ & Fp & $\mathrm{O}$ & Fl. \& Fr. Dec-Jan & $\mathrm{V}$ & GKR0637 \\
\hline \multicolumn{9}{|l|}{ PORTULACACEAE } \\
\hline Portulaca oleracea $\mathrm{L}$. & Nunia sag & G & $\mathrm{H}$ & Rs & $\mathrm{O}$ & $\begin{array}{l}\text { Fl.\& Fr. Through- } \\
\text { out the year }\end{array}$ & $\mathrm{V}$ & GKR0388 \\
\hline P. grandiflora Hook. & Time flower & M & $\mathrm{H}$ & Rs & $\mathrm{O}$ & $\begin{array}{l}\text { Fl. \& Fr. Through- } \\
\text { out the year }\end{array}$ & Or & GKR0126 \\
\hline \multicolumn{9}{|l|}{ BASELLACEAE } \\
\hline Basella alba $\mathrm{L}$. & Pui sak & $\begin{array}{l}\mathrm{D}, \mathrm{G}, \\
\mathrm{M}, \mathrm{T}\end{array}$ & $\mathrm{H}$ & $\mathrm{Ah}, \mathrm{Fp}$ & $\mathrm{C}$ & $\begin{array}{l}\text { Fl. Nov-Feb } \\
\text { Fr. U }\end{array}$ & $\mathrm{D}, \mathrm{V}$ & GKR2614 \\
\hline $\begin{array}{l}\text { MOLLUGINACEAE } \\
\text { Glinus lotoides L. }\end{array}$ & Duserasag & $\begin{array}{l}\mathrm{D}, \mathrm{G}, \\
\mathrm{M}, \mathrm{T}\end{array}$ & $\mathrm{H}$ & $\begin{array}{l}\text { Ah, Fp, } \\
\text { Rs }\end{array}$ & $\mathrm{C}$ & $\begin{array}{l}\text { Fl. Apr-May } \\
\text { Fr. U }\end{array}$ & $\mathrm{V}$ & GKR0173 \\
\hline $\begin{array}{l}\text { G. oppositifolius (L.) Aug. } \\
\text { DC. }\end{array}$ & Gima-sak & $\begin{array}{l}\mathrm{D}, \mathrm{G}, \\
\mathrm{M}, \mathrm{T}\end{array}$ & $\mathrm{H}$ & $\begin{array}{l}\text { Ah, Fp, } \\
\text { Rs }\end{array}$ & $\mathrm{C}$ & $\begin{array}{l}\text { Fl. Feb-Apr } \\
\text { Fr. U }\end{array}$ & $\begin{array}{l}\mathrm{M}, \\
\mathrm{V}\end{array}$ & GKR0024 \\
\hline Mollugo pentaphylla $\mathrm{L}$. & Khetpapra & $\begin{array}{l}\mathrm{D}, \mathrm{M}, \\
\mathrm{T}\end{array}$ & $\mathrm{H}$ & $\begin{array}{l}\text { Ah, Fp, } \\
\text { Rs }\end{array}$ & $\mathrm{O}$ & $\begin{array}{l}\text { Fl. \& Fr. Through- } \\
\text { out the year }\end{array}$ & $\mathrm{W}$ & GKR1110 \\
\hline
\end{tabular}

(Table contd.) 


\begin{tabular}{|c|c|c|c|c|c|c|c|c|}
\hline Scientific Name & Bangla name & District & Habit & Habitat & Occ. & Fl./Fr. Time & Use & RSE \\
\hline \multicolumn{9}{|l|}{ CARYOPHYLLACEAE } \\
\hline Dianthus caryophyllus L. & Jatasalpar & $\mathrm{G}, \mathrm{M}$ & $\mathrm{H}$ & Fp, Rs & $\mathrm{O}$ & $\begin{array}{l}\text { Fl.Nov-Jan } \\
\text { Fr. U }\end{array}$ & Or & GKR2903 \\
\hline $\begin{array}{l}\text { Polycarpon prostratum } \\
\text { (Forssk.) Aschers. \& } \\
\text { Scheinf. }\end{array}$ & Gima & $\mathrm{G}, \mathrm{M}$ & $\mathrm{H}$ & Fp, Rs & $\mathrm{O}$ & $\begin{array}{l}\text { Fl. Mar-Jun } \\
\text { Fr. Aug-Sep }\end{array}$ & $\mathrm{W}$ & GKR0178 \\
\hline Stellaria wallichiana Haines & $\begin{array}{l}\text { Commom } \\
\text { chickweed }\end{array}$ & G & $\mathrm{H}$ & $\mathrm{Ah}$ & $\mathrm{O}$ & $\begin{array}{l}\text { Fl. Feb-Aug } \\
\text { Fr. U }\end{array}$ & M & GKR2037 \\
\hline \multicolumn{9}{|l|}{ POLYGONACEAE } \\
\hline $\begin{array}{l}\text { Persicaria assamica } \\
\text { (Meisn.) Soják }\end{array}$ & Bishkathali & $\mathrm{D}$ & $\mathrm{H}$ & Rs & $\mathrm{O}$ & $\begin{array}{l}\text { Fl. Jun-Sep } \\
\text { Fr. U }\end{array}$ & M & GKR0154 \\
\hline P. barbata (L.) H.Hara & Bishkathali & $\mathrm{M}, \mathrm{T}$ & $\mathrm{H}$ & $\mathrm{Fp}, \mathrm{Rs}$ & $\mathrm{O}$ & $\begin{array}{l}\text { Fl. Nov-Feb } \\
\text { Fr. U }\end{array}$ & M & GKR0169 \\
\hline P. hydropiper (L.) Delarbre & Bishkatali & $\mathrm{D}, \mathrm{M}$ & $\mathrm{H}$ & $\mathrm{Fp}, \mathrm{Rs}$ & $\mathrm{O}$ & $\begin{array}{l}\text { Fl. Mar-Jul } \\
\text { Fr. U }\end{array}$ & M & GKR0428 \\
\hline P. lanata (Roxb.) Tzvelev & Bishkatali & $\mathrm{D}$ & $\mathrm{H}$ & Ah, Rs & $\mathrm{O}$ & $\begin{array}{l}\text { Fl. Jun-Sep } \\
\text { Fr. U }\end{array}$ & $\mathrm{W}$ & GKR1363 \\
\hline P. minor (Huds.) Opiz. & Bishkatali & $\mathrm{M}, \mathrm{T}$ & $\mathrm{H}$ & Rs & $\mathrm{O}$ & $\begin{array}{l}\text { Fl. Jan-Mar } \\
\text { Fr. U }\end{array}$ & $\mathrm{W}$ & GKR0116 \\
\hline P. orientalis (L.) Spach & $\begin{array}{l}\text { Bara } \\
\text { Panimarich }\end{array}$ & $\mathrm{D}, \mathrm{G}, \mathrm{T}$ & $\mathrm{H}$ & $\mathrm{Fp}, \mathrm{Rs}$ & $\mathrm{O}$ & $\begin{array}{l}\text { Fl. Jun-Oct } \\
\text { Fr. U }\end{array}$ & $\mathrm{W}$ & GKR0442 \\
\hline Polygonum chinense $\mathrm{L}$. & Bishkatali & $\begin{array}{l}\mathrm{D}, \mathrm{G} \\
\mathrm{M}, \mathrm{T}\end{array}$ & $\mathrm{H}$ & $\begin{array}{l}\text { Ah, Fp, } \\
\text { Rs }\end{array}$ & $\mathrm{C}$ & $\begin{array}{l}\text { Fl. Nov-Mar } \\
\text { Fr. U }\end{array}$ & M & GKR1843 \\
\hline P. effusum Meissn. & Chemti sag & $\mathrm{D}$ & $\mathrm{H}$ & Ah, Rs & $\mathrm{O}$ & $\begin{array}{l}\text { Fl. Mar-Oct } \\
\text { Fr. U }\end{array}$ & $\mathrm{W}$ & GKR1029 \\
\hline P. plebeium $\mathrm{R}$. Br. & $\begin{array}{l}\text { Khudi } \\
\text { bisakamtali }\end{array}$ & $\begin{array}{l}\mathrm{D}, \mathrm{G}, \\
\mathrm{M}, \mathrm{T}\end{array}$ & $\mathrm{H}$ & $\begin{array}{l}\text { Ah, Fp, } \\
\text { Rs }\end{array}$ & $\mathrm{C}$ & $\begin{array}{l}\text { Fl. Mar-Apr } \\
\text { Fr. U }\end{array}$ & $\mathrm{W}$ & GKR0586 \\
\hline P. flaccidum Meisn. & Lalbishkatali & $\mathrm{D}, \mathrm{M}$ & $\mathrm{H}$ & $\mathrm{Fp}, \mathrm{Rs}$ & $\mathrm{O}$ & $\begin{array}{l}\text { Fl. Aug-Oct } \\
\text { Fr. U }\end{array}$ & M & GKR2372 \\
\hline Rumex dentatus $\mathrm{L}$. & Banpalong & $\mathrm{D}$ & $\mathrm{H}$ & $\mathrm{Ah}, \mathrm{Fp}$ & $\mathrm{O}$ & $\begin{array}{l}\text { Fl. May-Jun } \\
\text { Fr. U }\end{array}$ & $\mathrm{W}$ & GKR0446 \\
\hline R. maritimus $\mathrm{L}$. & Gang palong & $\mathrm{D}, \mathrm{G}$ & $\mathrm{H}$ & $\begin{array}{l}\text { Ah, Fp, } \\
\text { Rs }\end{array}$ & $\mathrm{O}$ & $\begin{array}{l}\text { Fl. Feb-May } \\
\text { Fr. U }\end{array}$ & $\mathrm{W}$ & GKR0515 \\
\hline \multicolumn{9}{|l|}{ PLUMBAGINACEAE } \\
\hline Plumbago zeylanica $\mathrm{L}$. & Chitrak, Chita & $\mathrm{D}$ & $\mathrm{S}$ & $\mathrm{Ah}$ & $\mathrm{O}$ & $\begin{array}{l}\text { Fl. Nov-Mar } \\
\text { Fr. U }\end{array}$ & M & GKR1266 \\
\hline \multicolumn{9}{|l|}{ DILLENIACEAE } \\
\hline Dillenia indica $\mathrm{L}$. & Chalta & $\begin{array}{l}\mathrm{D}, \mathrm{G}, \\
\mathrm{M}, \mathrm{T}\end{array}$ & $\mathrm{T}$ & $\mathrm{Ah}, \mathrm{Bb}$ & $\mathrm{C}$ & $\begin{array}{l}\text { Fl. Jun-Aug } \\
\text { Fr. U }\end{array}$ & $\begin{array}{l}\mathrm{Fr} \\
\mathrm{M}\end{array}$ & GKR1057 \\
\hline \multicolumn{9}{|l|}{ ELAEOCARPACEAE } \\
\hline $\begin{array}{l}\text { Elaeocarpus floribundus } \\
\text { Blume }\end{array}$ & Jalpai & $\begin{array}{l}\mathrm{D}, \mathrm{G}, \\
\mathrm{M}, \mathrm{T}\end{array}$ & $\mathrm{T}$ & $\begin{array}{l}\text { Fp, Hy, } \\
\text { Rs }\end{array}$ & $\mathrm{O}$ & $\begin{array}{l}\text { Fl. Jul-Aug } \\
\text { Fr. U }\end{array}$ & $\begin{array}{l}\mathrm{Fr}, \\
\mathrm{O}\end{array}$ & GKR0067 \\
\hline E. tectorius (Lour.) Poir & & $\mathrm{T}$ & $\mathrm{T}$ & $\mathrm{Fp}$ & $\mathrm{O}$ & $\begin{array}{l}\text { Fl. May-Jun, } \\
\text { Fr. Aug-Oct }\end{array}$ & $\mathrm{Fr}, \mathrm{O}$ & GKR1496 \\
\hline \multicolumn{9}{|l|}{ TILIACEAE } \\
\hline Corchorus aestuans L. & Banpat & $\mathrm{D}, \mathrm{G}$ & $\mathrm{H}$ & Fp & $\mathrm{O}$ & $\begin{array}{l}\text { Fl. Aug-Feb } \\
\text { Fr. U }\end{array}$ & We & GKR0239 \\
\hline Grewia tenax (Forssk.) Fiori & Chhoto Assar & $\mathrm{D}, \mathrm{M}$ & $\mathrm{S}$ & $\mathrm{Fp}, \mathrm{Rs}$ & $\mathrm{O}$ & $\begin{array}{l}\text { Fl. Feb-Aug } \\
\text { Fr. U }\end{array}$ & $\mathrm{H}$ & GKR1275 \\
\hline
\end{tabular}




\begin{tabular}{|c|c|c|c|c|c|c|c|c|}
\hline Scientific Name & Bangla name & District & Habit & Habitat & Occ. & Fl./Fr. Time & Use & RSE \\
\hline Triumfetta rhomboidea Jacq. & Banokra & $\begin{array}{l}\mathrm{D}, \mathrm{G}, \\
\mathrm{M}, \mathrm{T}\end{array}$ & $\mathrm{S}$ & $\begin{array}{l}\text { Ah, Fp, } \\
\text { Rs }\end{array}$ & $\mathrm{C}$ & $\begin{array}{l}\text { Fl. Sep-Nov } \\
\text { Fr. U }\end{array}$ & $\begin{array}{l}\mathrm{Fb}, \\
\mathrm{M}\end{array}$ & GKR0305 \\
\hline STERCULIACEAE & & & & & & & & \\
\hline $\begin{array}{l}\text { Helicteres isora } \mathrm{L} \text {. } \\
\text { MALVACEAE }\end{array}$ & Rajot, Atmora & G & $\mathrm{S}$ & $\mathrm{Fp}$ & $\mathrm{O}$ & Fl. \& Fr. Sep-Mar & M & GKR0332 \\
\hline $\begin{array}{l}\text { Abelmoschus moschatus } \\
\text { Medik. }\end{array}$ & $\begin{array}{l}\text { Mushakdana, } \\
\text { musk okra }\end{array}$ & $\mathrm{D}$ & $\mathrm{H}$ & $\mathrm{Ah}, \mathrm{Fp}$ & $\mathrm{O}$ & $\begin{array}{l}\text { Fl. Jun-Oct } \\
\text { Fr. U }\end{array}$ & M & GKR1390 \\
\hline Abroma augusta (L.) L.f. & Ulat kambal & $\mathrm{T}$ & $\mathrm{S}$ & $\mathrm{Ah}, \mathrm{Fp}$ & $\mathrm{CE}$ & $\begin{array}{l}\text { Fl. Jun-Oct } \\
\text { Fr. U }\end{array}$ & M & GKR2090 \\
\hline Abutilon indicum (L.) Sweet & Potari & $\begin{array}{l}\mathrm{D}, \mathrm{M}, \\
\mathrm{T}\end{array}$ & $\mathrm{S}$ & $\begin{array}{l}\text { Ah, Fp, } \\
\text { Rs }\end{array}$ & $\mathrm{O}$ & $\begin{array}{l}\text { Fl. Sep-Apr } \\
\text { Fr. U }\end{array}$ & Or & GKR0140 \\
\hline Bombax ceiba $\mathrm{L}$. & Shimul & $\begin{array}{l}\mathrm{D}, \mathrm{G}, \\
\mathrm{M}, \mathrm{T}\end{array}$ & $\mathrm{T}$ & $\mathrm{Fp}, \mathrm{Rs}$ & $\mathrm{C}$ & $\begin{array}{l}\text { Fl. Jan-Mar } \\
\text { Fr. U }\end{array}$ & $\mathrm{Fb}, \mathrm{T}$ & GKR1006 \\
\hline Corchorus capsularis L. & Pat & $\mathrm{D}$ & $\mathrm{S}$ & $\begin{array}{l}\text { Ah, Fp, } \\
\text { Rs }\end{array}$ & $\mathrm{O}$ & $\begin{array}{l}\text { Fl. Oct-Dec, } \\
\text { Fr. Feb-Mar }\end{array}$ & $\mathrm{Fb}$ & GKR0556 \\
\hline Fioria vitifolia (L.) Mattei & Ban-carpus & $\mathrm{D}$ & $\mathrm{S}$ & $\mathrm{Fp}$ & $\mathrm{O}$ & Fl. Fr. Apr-Dec & $\mathrm{W}$ & GKR1662 \\
\hline Gossypium arboreum $\mathrm{L}$. & Karpas & M & $\mathrm{T}$ & Fp & $\mathrm{O}$ & $\begin{array}{l}\text { Fl. Jun-Jul } \\
\text { Fr. U }\end{array}$ & $\mathrm{Fb}$ & GKR2623 \\
\hline Grewia multiflora Juss. & Panisara & $\mathrm{D}, \mathrm{T}$ & ST & Rs & $\mathrm{O}$ & $\begin{array}{l}\text { Fl. Jul-Sep } \\
\text { Fr. U }\end{array}$ & $\begin{array}{l}\text { Fw, } \\
\mathrm{W}\end{array}$ & GKR1335 \\
\hline G. nervosa (Lour.) Panigrahi & Assar/ Datoi & G & ST & Rs & $\mathrm{O}$ & $\begin{array}{l}\text { Fl. Aug-Apr } \\
\text { Fr. U }\end{array}$ & $\begin{array}{l}\text { Fw, } \\
\text { M }\end{array}$ & GKR0744 \\
\hline Hibiscus rosa-sinensis $\mathrm{L}$. & Joba & $\begin{array}{l}\mathrm{D}, \mathrm{G}, \\
\mathrm{M}, \mathrm{T}\end{array}$ & $\mathrm{S}$ & $\mathrm{Ah}, \mathrm{Fp}$ & $\mathrm{C}$ & $\begin{array}{l}\text { Fl. \& Fr. Through- } \\
\text { out the year }\end{array}$ & Or & GKR1526 \\
\hline H. sabdariffa $\mathrm{L}$. & $\begin{array}{l}\text { Chukair, } \\
\text { Chukur }\end{array}$ & G & $\mathrm{S}$ & Rs & $\mathrm{O}$ & $\begin{array}{l}\text { Fl. Apr-Jul } \\
\text { Fr. U }\end{array}$ & $\begin{array}{l}\mathrm{Fb} \\
\mathrm{V}\end{array}$ & GKR1954 \\
\hline $\begin{array}{l}\text { Malvastrum } \\
\text { coromandelianum (L.) } \\
\text { Gracke }\end{array}$ & Broom weed & M & $\mathrm{H}$ & Rs & $\mathrm{O}$ & $\begin{array}{l}\text { Fl. Mar-Sep } \\
\text { Fr. U }\end{array}$ & M & GKR1247 \\
\hline Melochia corchorifolia $\mathrm{L}$. & Banpat & $\begin{array}{l}\mathrm{D}, \mathrm{G}, \\
\mathrm{M}, \mathrm{T}\end{array}$ & $\mathrm{S}$ & $\begin{array}{l}\text { Ah, Fp, } \\
\text { Rs }\end{array}$ & $\mathrm{C}$ & $\begin{array}{l}\text { Fl. Jul-Apr } \\
\text { Fr. U }\end{array}$ & $\mathrm{Fb}$ & GKR0059 \\
\hline Pentapetes phoenicea $\mathrm{L}$. & Morich joba & $\begin{array}{l}\mathrm{D}, \mathrm{G}, \\
\mathrm{M}, \mathrm{T}\end{array}$ & $\mathrm{H}$ & $\begin{array}{l}\text { Ah, Fp, } \\
\text { Rs }\end{array}$ & $\mathrm{C}$ & Fl. \& Fr. Jun-Jan & $\begin{array}{l}\mathrm{Fb}, \\
\mathrm{Or}\end{array}$ & GKR0204 \\
\hline Sida acuta Burm. f. & Kureta & $\begin{array}{l}\mathrm{D}, \mathrm{G}, \\
\mathrm{M}, \mathrm{T}\end{array}$ & $\mathrm{H}$ & $\begin{array}{l}\text { Ah, Fp, } \\
\text { Rs }\end{array}$ & $\mathrm{C}$ & $\begin{array}{l}\text { Fl. Sep-May } \\
\text { Fr. U }\end{array}$ & M & GKR0184 \\
\hline S. cordifolia $\mathrm{L}$. & Jhunka & M & $\mathrm{H}$ & Rs & $\mathrm{O}$ & $\begin{array}{l}\text { Fl.\& Fr. Through- } \\
\text { out the year }\end{array}$ & M & GKR1824 \\
\hline S. mysorensis Wight \& Arn. & Berela & G & $\mathrm{H}$ & Fp, Rs & $\mathrm{O}$ & $\begin{array}{l}\text { Fl. Oct-Feb } \\
\text { Fr. U }\end{array}$ & M & GKR0242 \\
\hline S. rhombifolia $\mathrm{L}$. & Berela & $\begin{array}{l}\mathrm{D}, \mathrm{G}, \\
\mathrm{M}, \mathrm{T}\end{array}$ & $\mathrm{H}$ & $\mathrm{Fp}, \mathrm{Rs}$ & $\mathrm{C}$ & $\begin{array}{l}\text { Fl. Jul-Dec } \\
\text { Fr. U }\end{array}$ & M & GKR0287 \\
\hline Urena lobata $\mathrm{L}$. & Ghagra & $\mathrm{D}$ & $\mathrm{S}$ & Fp, Rs & $\mathrm{O}$ & $\begin{array}{l}\text { Fl. Jan-Apr } \\
\text { Fr. U }\end{array}$ & $\mathrm{W}$ & GKR0081 \\
\hline \multicolumn{9}{|l|}{ LECYTHIDACEAE } \\
\hline $\begin{array}{l}\text { Barringtonia acutangula } \\
\text { (L.) Gaertn. }\end{array}$ & Hijal & $\begin{array}{l}\mathrm{D}, \mathrm{G}, \\
\mathrm{M}, \mathrm{T}\end{array}$ & $\mathrm{T}$ & $\begin{array}{l}\text { Ep, Fp, } \\
\text { Rs }\end{array}$ & $\mathrm{C}$ & $\begin{array}{l}\text { Fl. Mar-May } \\
\text { Fr. U }\end{array}$ & $\begin{array}{l}\mathrm{Bb}, \\
\mathrm{Cw}, \\
\mathrm{Rp}\end{array}$ & GKR0699 \\
\hline $\begin{array}{l}\text { CARICACEAE } \\
\text { Carica papaya } \mathrm{L} .\end{array}$ & Pepe & $\begin{array}{l}\mathrm{D}, \mathrm{G}, \\
\mathrm{M}\end{array}$ & $\mathrm{T}$ & $\mathrm{Ah}$ & $\mathrm{O}$ & $\begin{array}{l}\text { Fl.\& Fr. Through- } \\
\text { out the year }\end{array}$ & $\begin{array}{l}\mathrm{Fr} \\
\mathrm{M}, \mathrm{V}\end{array}$ & GKR0006 \\
\hline
\end{tabular}




\begin{tabular}{|c|c|c|c|c|c|c|c|c|}
\hline Scientific Name & Bangla name & District & Habit & Habitat & Occ. & Fl./Fr. Time & Use & RSE \\
\hline \multicolumn{9}{|l|}{ CUCURBITACEAE } \\
\hline $\begin{array}{l}\text { Bryonia cochinchinensis } \\
\text { Lour. }\end{array}$ & Boti-jhinga & G & $\mathrm{H}$ & Fp, Rs & $\mathrm{O}$ & $\begin{array}{l}\text { Fl. May-Jul } \\
\text { Fr. U }\end{array}$ & M & GKR1430 \\
\hline Coccinia grandis (L.) Voigt & Telakucha & $\begin{array}{l}\mathrm{D}, \mathrm{G}, \\
\mathrm{M}, \mathrm{T}\end{array}$ & $\mathrm{H}$ & $\begin{array}{l}\text { Ah, Fp, } \\
\text { Rs }\end{array}$ & $\mathrm{C}$ & $\begin{array}{l}\text { Fl. Dec-Mar } \\
\text { Fr. U }\end{array}$ & M & GKR1642 \\
\hline $\begin{array}{l}\text { Cucumis callosus (Rottler) } \\
\text { Cogn. }\end{array}$ & Tita Bangi & $\mathrm{D}$ & $\mathrm{H}$ & Fp, Rs & $\mathrm{O}$ & $\begin{array}{l}\text { Fl. Jul-Jan } \\
\text { Fr. U }\end{array}$ & $\mathrm{Fr}$ & GKR1369 \\
\hline C. sativus $\mathrm{L}$. & Sosha & $\mathrm{D}$ & $\mathrm{H}$ & $\mathrm{Fp}$ & $\mathrm{O}$ & $\begin{array}{l}\text { Fl. Sep-Mar } \\
\text { Fr. U }\end{array}$ & Fr, $\mathrm{V}$ & GKR1316 \\
\hline $\begin{array}{l}\text { Luffa cylindrica (L.) M. } \\
\text { Roem. }\end{array}$ & Dhundul & $\mathrm{D}$ & $\mathrm{H}$ & $\mathrm{Ah}$ & $\mathrm{O}$ & $\begin{array}{l}\text { Fl. Feb-Dec } \\
\text { Fr. U }\end{array}$ & $\mathrm{V}$ & GKR1348 \\
\hline Momordica charantia L. & Ucche & $\begin{array}{l}\mathrm{D}, \mathrm{M}, \\
\mathrm{T}\end{array}$ & $\mathrm{H}$ & $\begin{array}{l}\text { Ah, Fp, } \\
\text { Rs }\end{array}$ & $\mathrm{O}$ & $\begin{array}{l}\text { Fl. Jun-Sep } \\
\text { Fr. U }\end{array}$ & $\begin{array}{l}\mathrm{M}, \\
\mathrm{V}\end{array}$ & GKR0580 \\
\hline $\begin{array}{l}\text { M. cochinchinensis (Lour.) } \\
\text { Spreng. }\end{array}$ & Kakrol & G & $\mathrm{H}$ & $\mathrm{Ah}$ & $\mathrm{O}$ & $\begin{array}{l}\text { Fl. Jun-Aug } \\
\text { Fr. U }\end{array}$ & $\mathrm{V}$ & GKR0296 \\
\hline M. dioica Roxb. ex Willd. & Dharkarolla & $\begin{array}{l}\mathrm{D}, \mathrm{G} \\
\mathrm{M}\end{array}$ & $\mathrm{H}$ & $\begin{array}{l}\text { Ah, Fp, } \\
\text { Rs }\end{array}$ & $\mathrm{O}$ & $\begin{array}{l}\text { Fl. Jul-Dec } \\
\text { Fr. U }\end{array}$ & $\mathrm{V}$ & GKR0794 \\
\hline M. subangulata Blume & Kakrol & $\mathrm{D}, \mathrm{T}$ & $\mathrm{H}$ & $\mathrm{Ah}, \mathrm{Fp}$ & $\mathrm{O}$ & $\begin{array}{l}\text { Fl. Jun-Aug } \\
\text { Fr. U }\end{array}$ & $\mathrm{V}$ & GKR0662 \\
\hline $\begin{array}{l}\text { Mukia maderaspatana (L.) } \\
\text { M. Roem. }\end{array}$ & $\begin{array}{l}\text { Agmukhi, } \\
\text { Bilari }\end{array}$ & $\mathrm{D}, \mathrm{M}$ & $\mathrm{H}$ & $\mathrm{Fp}$ & $\mathrm{O}$ & $\begin{array}{l}\text { Fl. \& Fr. } \\
\text { Throughout the } \\
\text { year }\end{array}$ & M & GKR1647 \\
\hline Trichosanthes cordata Roxb. & $\begin{array}{l}\text { Chichinga, } \\
\text { Rekha }\end{array}$ & $\mathrm{D}$ & $\mathrm{H}$ & $\mathrm{Fp}$ & $\mathrm{O}$ & $\begin{array}{l}\text { Fl. Jul-Aug } \\
\text { Fr. U }\end{array}$ & $\mathrm{V}$ & GKR1688 \\
\hline T. dioica Roxb. & Potol & $\mathrm{T}$ & $\mathrm{H}$ & Rs & $\mathrm{O}$ & $\begin{array}{l}\text { Fl. Feb-Sep } \\
\text { Fr. U }\end{array}$ & $\mathrm{V}$ & GKR1557 \\
\hline T. tricuspidata Lour. & Makal & M & $\mathrm{H}$ & $\mathrm{Ah}$ & $\mathrm{O}$ & $\begin{array}{l}\text { Fl. \& Fr. Through- } \\
\text { out the year }\end{array}$ & $\mathrm{W}$ & GKR1394 \\
\hline $\begin{array}{l}\text { Zehneria japonica (Thunb.) } \\
\text { H.Y. Liu }\end{array}$ & Rakhalshasha & M & $\mathrm{H}$ & Rs & $\mathrm{O}$ & $\begin{array}{l}\text { Fl. Apr-Jul } \\
\text { Fr. U }\end{array}$ & M & GKR0084 \\
\hline $\begin{array}{l}\text { Z. thwaitesii (Schweinf.) } \\
\text { C.Jeffrey }\end{array}$ & Unknown & M & $\mathrm{H}$ & $\mathrm{Ah}, \mathrm{Fp}$ & $\mathrm{O}$ & $\begin{array}{l}\text { Fl. Aug-Dec } \\
\text { Fr. U }\end{array}$ & $\mathrm{W}$ & GKR2503 \\
\hline \multicolumn{9}{|l|}{ SALICACEAE } \\
\hline $\begin{array}{l}\text { Flacourtia indica (Burm. f.) } \\
\text { Merr. }\end{array}$ & Beuchi, Katai & $\mathrm{D}, \mathrm{G}$ & $\mathrm{S}$ & Rs & $\mathrm{O}$ & Fl. \& Fr. Nov-Mar & $\mathrm{W}$ & GKR1535 \\
\hline F. jangomas (Lour.) Raeus. & Lukluki & $\begin{array}{l}\mathrm{D}, \mathrm{G}, \\
\mathrm{M}\end{array}$ & ST & $\mathrm{Ah}, \mathrm{Fp}$ & $\mathrm{O}$ & $\begin{array}{l}\text { Fl. Mar-Apr. } \\
\text { Fr. Aug-Oct }\end{array}$ & $\mathrm{Fr}$ & GKR0342 \\
\hline Salix tetrasperma Roxb. & $\begin{array}{l}\text { Boishakhi, } \\
\text { Panijama }\end{array}$ & M & $\mathrm{T}$ & $\mathrm{Ah}$ & $\mathrm{O}$ & $\begin{array}{l}\text { Fl. Jan-Feb } \\
\text { Fr. U }\end{array}$ & Fw & GKR0158 \\
\hline \multicolumn{9}{|l|}{ CAPPARACEAE } \\
\hline Capparis zeylanica $\mathrm{L}$. & Asarilata, & $\mathrm{G}, \mathrm{T}$ & $\mathrm{S}$ & $\begin{array}{l}\text { Ah, Fp, } \\
\text { Rs }\end{array}$ & $\mathrm{O}$ & $\begin{array}{l}\text { Fl. Mar-May } \\
\text { Fr. U }\end{array}$ & M & GKR0375 \\
\hline Cleome rutidosperma DC. & $\begin{array}{l}\text { Begunihurhure } \\
\text { y }\end{array}$ & $\mathrm{D}, \mathrm{G}$ & $\mathrm{H}$ & $\begin{array}{l}\text { Ah, Fp, } \\
\text { Rs }\end{array}$ & $\mathrm{O}$ & $\begin{array}{l}\text { Fl. May-Nov } \\
\text { Fr. U }\end{array}$ & $\mathrm{W}$ & GKR0491 \\
\hline Crateva magna (Lour.) DC. & Bonna & $\begin{array}{l}\mathrm{D}, \mathrm{G}, \\
\mathrm{M}, \mathrm{T}\end{array}$ & $\mathrm{T}$ & Ep, Rs & $\mathrm{C}$ & $\begin{array}{l}\text { Fl. Mar-Apr } \\
\text { Fr. U }\end{array}$ & Fw & GKR1528 \\
\hline $\begin{array}{l}\text { BRASSICACEAE } \\
\text { Brassica napus } \mathrm{L} \text {. }\end{array}$ & Sorisha & $\mathrm{G}, \mathrm{M}$ & $\mathrm{H}$ & $\mathrm{Ah}, \mathrm{Fp}$ & $\mathrm{O}$ & $\begin{array}{l}\text { Fl. Mar-Aug } \\
\text { Fr. U }\end{array}$ & $\mathrm{O}$ & GKR0151 \\
\hline
\end{tabular}

(Table contd.) 


\begin{tabular}{|c|c|c|c|c|c|c|c|c|}
\hline Scientific Name & Bangla name & District & Habit & Habitat & Occ. & Fl./Fr. Time & Use & RSE \\
\hline B. nigra (L.) K.Koch & $\begin{array}{l}\text { Kalo Sarisha, } \\
\text { Rai Sarisha }\end{array}$ & $\begin{array}{l}\mathrm{D}, \mathrm{G}, \\
\mathrm{M}\end{array}$ & $\mathrm{H}$ & $\mathrm{Ah}$ & $\mathrm{O}$ & $\begin{array}{l}\text { Fl. Mar-May } \\
\text { Fr. U }\end{array}$ & $\mathrm{O}$ & GKR0718 \\
\hline Rorippa indica (L.) Hiern & Bon Sorisa & $\begin{array}{l}\mathrm{D}, \mathrm{G}, \\
\mathrm{M}\end{array}$ & $\mathrm{H}$ & $\begin{array}{l}\text { Ah, Fp, } \\
\text { Rs }\end{array}$ & $\mathrm{O}$ & $\begin{array}{l}\text { Fl. Mar-Apr } \\
\text { Fr. U }\end{array}$ & $\mathrm{W}$ & GKR0455 \\
\hline \multicolumn{9}{|l|}{ MORINGACEAE } \\
\hline Moringa oleifera Lamk. & Sajna & $\mathrm{D}$ & $\mathrm{T}$ & $\begin{array}{l}\text { Ah, Ep, } \\
\text { Fp }\end{array}$ & $\mathrm{O}$ & $\begin{array}{l}\text { Fl. Jan-Mar } \\
\text { Fr. U }\end{array}$ & $\mathrm{M}, \mathrm{V}$ & GKR0572 \\
\hline \multicolumn{9}{|l|}{ SAPOTACEAE } \\
\hline Chrysophyllum cainito L. & Star apple & $\mathrm{D}, \mathrm{M}$ & $\mathrm{T}$ & Ah, Rs, & $\mathrm{O}$ & $\begin{array}{l}\text { Fl. Jul-Aug } \\
\text { Fr. U }\end{array}$ & $\begin{array}{l}\text { Fr, } \\
\text { Fw, } \\
\text { T }\end{array}$ & GKR1331 \\
\hline $\begin{array}{l}\text { Madhuca longifolia } \\
\text { (J.Koenig ex L.) J.F.Macbr. }\end{array}$ & Mohua & M & $\mathrm{T}$ & Hy & $\mathrm{O}$ & $\begin{array}{l}\text { Fl. Mar-May } \\
\text { Fr. U }\end{array}$ & M & GKR1932 \\
\hline $\begin{array}{l}\text { Manilkara zapota (L.) } \\
\text { P.Royen }\end{array}$ & Safeda & $\begin{array}{l}\mathrm{D}, \mathrm{G} \\
\mathrm{M}, \mathrm{T}\end{array}$ & $\mathrm{T}$ & $\mathrm{Ah}, \mathrm{Fp}$ & $\mathrm{C}$ & $\begin{array}{l}\text { Fl. Aug-Oct } \\
\text { Fr. U }\end{array}$ & $\mathrm{Fr}$ & GKR0118 \\
\hline Mimusops elengi $\mathrm{L}$. & Bokul & $\begin{array}{l}\mathrm{D}, \mathrm{G} \\
\mathrm{M}, \mathrm{T}\end{array}$ & $\mathrm{T}$ & $\mathrm{Ah}$ & $\mathrm{C}$ & $\begin{array}{l}\text { Fl. Mar-Jun } \\
\text { Fr. U }\end{array}$ & Or & GKR1702 \\
\hline \multicolumn{9}{|l|}{ EBENACEAE } \\
\hline Diospyros discolor Willd. & Bilati gab & $\begin{array}{l}\mathrm{D}, \mathrm{G}, \\
\mathrm{M}, \mathrm{T}\end{array}$ & $\mathrm{H}$ & $\begin{array}{l}\mathrm{Bb}, \mathrm{Fp} \\
\mathrm{Rs}\end{array}$ & $\mathrm{C}$ & $\begin{array}{l}\text { Fl. May-Aug } \\
\text { Fr. U }\end{array}$ & $\mathrm{Fr}, \mathrm{T}$ & GKR0034 \\
\hline D. montana Roxb. & Tomal & $\mathrm{D}, \mathrm{T}$ & $\mathrm{T}$ & $\mathrm{Fp}, \mathrm{Rs}$ & $\mathrm{O}$ & $\begin{array}{l}\text { Fl. Mar-Apr } \\
\text { Fr. U }\end{array}$ & $\mathrm{T}$ & GKR0658 \\
\hline $\begin{array}{l}\text { D. malabarica (Desr.) } \\
\text { Kostel. }\end{array}$ & Deshi gab & $\begin{array}{l}\mathrm{D}, \mathrm{G} \\
\mathrm{M}, \mathrm{T}\end{array}$ & $\mathrm{T}$ & $\begin{array}{l}\mathrm{Ah}, \\
\mathrm{Bb}, \mathrm{Fp}\end{array}$ & $\mathrm{C}$ & $\begin{array}{l}\text { Fl. May-Jun } \\
\text { Fr. U }\end{array}$ & $\begin{array}{l}\text { Fr, } \\
\text { M, T }\end{array}$ & GKR0715 \\
\hline $\begin{array}{l}\text { D. peregrina (Gaertn.) } \\
\text { Gürke }\end{array}$ & Gab & M & $\mathrm{T}$ & $\mathrm{Ah}$ & $\mathrm{O}$ & $\begin{array}{l}\text { Fl. Jan-Feb } \\
\text { Fr. U }\end{array}$ & $\mathrm{D}, \mathrm{M}$ & GKR1586 \\
\hline D. philippinensis A.DC & Bilatigab & $\mathrm{D}, \mathrm{T}$ & $\mathrm{T}$ & $\mathrm{Fp}, \mathrm{Rs}$ & $\mathrm{O}$ & $\begin{array}{l}\text { Fl. Apr-Jun } \\
\text { Fr. U }\end{array}$ & Fr & GKR1498 \\
\hline $\begin{array}{l}\text { MYRSINACEAE } \\
\text { Ardisia humilis Vahl }\end{array}$ & Banjam & $\mathrm{G}, \mathrm{M}$ & S & Fp, Rs & $\mathrm{O}$ & $\begin{array}{l}\text { Fl. Mar-Dec } \\
\text { Fr. U }\end{array}$ & M & GKR0019 \\
\hline $\begin{array}{l}\text { PRIMULACEAE } \\
\text { Androsace umbellata (Lour.) } \\
\text { Merr. }\end{array}$ & Unknown & $\mathrm{D}, \mathrm{M}$ & $\mathrm{H}$ & Rs & $\mathrm{O}$ & $\begin{array}{l}\text { Fl. Feb-Apr } \\
\text { Fr. U }\end{array}$ & $\mathrm{W}$ & GKR0125 \\
\hline $\begin{array}{l}\text { CRASSULACEAE } \\
\text { Kalanchoe pinnata (Lam.) } \\
\text { Pers. }\end{array}$ & Patharkuchi & $\begin{array}{l}\mathrm{D}, \mathrm{G} \\
\mathrm{M}\end{array}$ & $\mathrm{H}$ & $\mathrm{Ah}$ & $\mathrm{O}$ & $\begin{array}{l}\text { Fl. Jan-Mar } \\
\text { Fr. U }\end{array}$ & M & GKR2490 \\
\hline \multicolumn{9}{|l|}{ ROSACEAE } \\
\hline Rosa chinensis Jacq. & Golap & $\mathrm{D}$ & $S$ & $\mathrm{Ah}$ & $\mathrm{O}$ & $\begin{array}{l}\text { Fl. Apr-Sep } \\
\text { Fr. U }\end{array}$ & Or & GKR1526 \\
\hline $\begin{array}{l}\text { MIMOSACEAE } \\
\text { Mimosa pudica L. }\end{array}$ & Lajjabati & $\mathrm{D}, \mathrm{G}$ & $\mathrm{H}$ & Fp, Rs & $\mathrm{O}$ & $\begin{array}{l}\text { Fl. Nov-Mar } \\
\text { Fr. U }\end{array}$ & M & GKR0414 \\
\hline $\begin{array}{l}\text { CAESALPINIACEAE } \\
\text { Senna occidentalis } \text { (L.) Link }\end{array}$ & $\begin{array}{l}\text { Barakalkasund } \\
\text { a }\end{array}$ & $\mathrm{D}, \mathrm{G}$ & $S$ & $\mathrm{Fp}, \mathrm{Rs}$ & $\mathrm{O}$ & $\begin{array}{l}\text { Fl. \& Fr. Through- } \\
\text { out the year }\end{array}$ & M & GKR0721 \\
\hline S. sophera (L.) Roxb. & $\begin{array}{l}\text { Chhotokalkasu } \\
\text { nda }\end{array}$ & $\mathrm{D}, \mathrm{G}$ & $\mathrm{H}$ & $\mathrm{Fp}, \mathrm{Rs}$ & $\mathrm{O}$ & $\begin{array}{l}\text { Fl. Nov-Feb } \\
\text { Fr. U }\end{array}$ & M & GKR0232 \\
\hline S. tora (L.) Roxb. & Kalkasunda & $\begin{array}{l}\mathrm{D}, \mathrm{G}, \\
\mathrm{M}, \mathrm{T}\end{array}$ & $\mathrm{H}$ & $\mathrm{Fp}, \mathrm{Rs}$ & $\mathrm{C}$ & $\begin{array}{l}\text { Fl. \& Fr. Through- } \\
\text { out the year }\end{array}$ & M & GKR1118 \\
\hline
\end{tabular}

(Table contd.) 


\begin{tabular}{|c|c|c|c|c|c|c|c|c|}
\hline Scientific Name & Bangla name & District & Habit & Habitat & Occ. & Fl./Fr. Time & Use & RSE \\
\hline \multicolumn{9}{|l|}{ FABACEAE } \\
\hline Acacia auriculiformis Benth. & Akashmoni & $\begin{array}{l}\mathrm{D}, \mathrm{G}, \\
\mathrm{M}\end{array}$ & $\mathrm{T}$ & $\mathrm{Fp}, \mathrm{Rs}$ & $\mathrm{O}$ & $\begin{array}{l}\text { Fl. Dec-Mar } \\
\text { Fr. U }\end{array}$ & $\mathrm{T}$ & GKR1067 \\
\hline A. nilotica (L.) Delile & Babla & $\mathrm{G}, \mathrm{M}$ & $\mathrm{T}$ & $\mathrm{Fp}, \mathrm{Rs}$ & $\mathrm{O}$ & $\begin{array}{l}\text { Fl. Jul-Dec } \\
\text { Fr. U }\end{array}$ & M & GKR2014 \\
\hline Albizia lebbeck (L.) Benth. & $\begin{array}{l}\text { Sirish, } \\
\text { Kalokoroi }\end{array}$ & $\begin{array}{l}\mathrm{D}, \mathrm{G}, \\
\mathrm{M}\end{array}$ & $\mathrm{T}$ & Ah, Rs & $\mathrm{O}$ & $\begin{array}{l}\text { Fl. Mar-May, } \\
\text { Fr.- Aug-Oct }\end{array}$ & $\mathrm{T}$ & GKR0508 \\
\hline A. lucida Benth. & $\begin{array}{l}\text { Motor Koroi, } \\
\text { Potka Siris }\end{array}$ & $\mathrm{D}, \mathrm{G}$ & $\mathrm{T}$ & Rs & $\mathrm{O}$ & $\begin{array}{l}\text { Fl. Jun-Jul } \\
\text { Fr. U }\end{array}$ & $\begin{array}{l}\text { T, } \\
\text { Fw }\end{array}$ & GKR0336 \\
\hline A. procera (Roxb.) Benth. & $\begin{array}{l}\text { Sada } \\
\text { Siris,Koroi }\end{array}$ & $\begin{array}{l}\mathrm{D}, \mathrm{G}, \\
\mathrm{M}, \mathrm{T}\end{array}$ & $\mathrm{T}$ & Fp, Rs & $\mathrm{C}$ & $\begin{array}{l}\text { Fl. Sep-Feb } \\
\text { Fr. U }\end{array}$ & $\mathrm{T}$ & GKR0641 \\
\hline $\begin{array}{l}\text { A. richardiana (Voigt) King } \\
\text { \& Prain }\end{array}$ & $\begin{array}{l}\text { Gagansirish, } \\
\text { Rajkoroi }\end{array}$ & $\begin{array}{l}\mathrm{D}, \mathrm{G}, \\
\mathrm{M}, \mathrm{T}\end{array}$ & $\mathrm{T}$ & $\mathrm{Fp}, \mathrm{Rs}$ & $\mathrm{C}$ & $\begin{array}{l}\text { Fl. Mar-May } \\
\text { Fr. U }\end{array}$ & $\mathrm{T}$ & GKR1705 \\
\hline A. saman (Jacq.) Merr. & Raintree & $\begin{array}{l}\mathrm{D}, \mathrm{G}, \\
\mathrm{M}, \mathrm{T}\end{array}$ & $\mathrm{T}$ & $\mathrm{Fp}, \mathrm{Rs}$ & $\mathrm{C}$ & $\begin{array}{l}\text { Fl. Mar-Jun } \\
\text { Fr. U }\end{array}$ & $\mathrm{T}$ & GKR1246 \\
\hline $\begin{array}{l}\text { Alysicarpus ovalifolius } \\
\text { (Schum.) Leonard }\end{array}$ & $\begin{array}{l}\text { False } \\
\text { moneywort }\end{array}$ & $\mathrm{T}$ & $\mathrm{H}$ & Fp, Rs & $\mathrm{O}$ & $\begin{array}{l}\text { Fl. Jun-Sep } \\
\text { Fr. U }\end{array}$ & W & GKR1071 \\
\hline Arachis hypogea $\mathrm{L}$. & Cheenabadam & $\mathrm{D}$ & $\mathrm{H}$ & Fp & $\mathrm{O}$ & $\begin{array}{l}\text { Fl. Jul-Aug } \\
\text { Fr. U }\end{array}$ & $\mathrm{O}$ & GKR1367 \\
\hline Bauhinia acuminata $\mathrm{L}$. & kanchan & $\begin{array}{l}\mathrm{D}, \mathrm{G}, \\
\mathrm{M}, \mathrm{T}\end{array}$ & $\mathrm{S}$ & $\mathrm{Fp}, \mathrm{Rs}$ & $\mathrm{C}$ & $\begin{array}{l}\text { Fl. Apr-May } \\
\text { Fr. U }\end{array}$ & M & GKR1525 \\
\hline $\begin{array}{l}\text { Butea monosperma (Lam.) } \\
\text { Taub. }\end{array}$ & Palash & $\begin{array}{l}\mathrm{G}, \mathrm{M}, \\
\mathrm{T}\end{array}$ & $\mathrm{T}$ & $\mathrm{Ah}$ & $\mathrm{O}$ & $\begin{array}{l}\text { Fl. Feb-Apr } \\
\text { Fr. U }\end{array}$ & $\begin{array}{l}\mathrm{M}, \\
\mathrm{Re}\end{array}$ & GKR2047 \\
\hline Cajanus cajan (L.) Millsp. & Arhar & $\begin{array}{l}\mathrm{G}, \mathrm{M}, \\
\mathrm{T}\end{array}$ & $\mathrm{H}$ & Ah, Rs & $\mathrm{O}$ & $\begin{array}{l}\text { Fl. Dec-Mar } \\
\text { Fr. U }\end{array}$ & $\mathrm{C}$ & GKR0164 \\
\hline $\begin{array}{l}\text { Canavalia ensiformis }(\mathrm{L} .) \\
\text { DC. }\end{array}$ & Jack bean & $\mathrm{D}$ & $\mathrm{H}$ & $\mathrm{Ah}$ & $\mathrm{O}$ & $\begin{array}{l}\text { Fl. May-Oct } \\
\text { Fr. U }\end{array}$ & $\mathrm{V}$ & GKR1630 \\
\hline Cassia fistula $\mathrm{L}$. & $\begin{array}{l}\text { Sonalu, Bador } \\
\text { lathi }\end{array}$ & $\begin{array}{l}\mathrm{D}, \mathrm{G}, \\
\mathrm{M}, \mathrm{T}\end{array}$ & $\mathrm{H}$ & $\mathrm{Fp}$ & $\mathrm{C}$ & $\begin{array}{l}\text { Fl. Mar-Sep } \\
\text { Fr. U }\end{array}$ & $\begin{array}{l}\mathrm{M}, \\
\text { Or, } \\
\mathrm{T}\end{array}$ & GKR1564 \\
\hline Clitoria ternatea $\mathrm{L}$. & Oporazita & M & $\mathrm{H}$ & Rs & $\mathrm{O}$ & $\begin{array}{l}\text { Fl. Mar-May } \\
\text { Fr. U }\end{array}$ & $\begin{array}{l}\mathrm{M}, \\
\text { Or }\end{array}$ & GKR1839 \\
\hline Crotalaria juncea $\mathrm{L}$. & Jhunjuni & $\mathrm{D}, \mathrm{T}$ & $\mathrm{H}$ & Rs & $\mathrm{O}$ & $\begin{array}{l}\text { Fl. Jul-Sep } \\
\text { Fr. U }\end{array}$ & $\mathrm{Fb}$ & GKR1678 \\
\hline C. pallida Ait. & Jhunjuni & $\mathrm{D}, \mathrm{G}, \mathrm{T}$ & $\mathrm{H}$ & $\mathrm{Fp}, \mathrm{Rs}$ & $\mathrm{O}$ & $\begin{array}{l}\text { Fl. Sep-Jan } \\
\text { Fr. U }\end{array}$ & W & GKR1675 \\
\hline Dalbergia sissoo DC. & Sissoo & $\begin{array}{l}\mathrm{D}, \mathrm{G}, \\
\mathrm{M}, \mathrm{T}\end{array}$ & $\mathrm{T}$ & $\mathrm{Fp}, \mathrm{Rs}$ & $\mathrm{C}$ & $\begin{array}{l}\text { Fl. Mar-Jul } \\
\text { Fr. U }\end{array}$ & $\mathrm{T}$ & GKR1673 \\
\hline Delonix regia (Hook.) Raf. & Krishnachura & $\begin{array}{l}\mathrm{D}, \mathrm{G}, \\
\mathrm{M}, \mathrm{T}\end{array}$ & $\mathrm{T}$ & $\begin{array}{l}\mathrm{Bb}, \mathrm{Fp}, \\
\mathrm{Rs}\end{array}$ & $\mathrm{C}$ & $\begin{array}{l}\text { Fl. Mar-Oct } \\
\text { Fr. U }\end{array}$ & Or & GKR1089 \\
\hline $\begin{array}{l}\text { Desmodium gangeticum (L.) } \\
\text { DC. }\end{array}$ & Salpani & $\begin{array}{l}\mathrm{D}, \mathrm{G}, \\
\mathrm{M}, \mathrm{T}\end{array}$ & $\mathrm{H}$ & $\begin{array}{l}\text { Ah, Fp, } \\
\text { Rs }\end{array}$ & $\mathrm{C}$ & $\begin{array}{l}\text { Fl. Oct-Dec } \\
\text { Fr. U }\end{array}$ & M & GKR0299 \\
\hline D. motorium (Houtt.) Merr. & Turup Chandal & M & $\mathrm{H}$ & $\mathrm{Fp}$ & $\mathrm{O}$ & $\begin{array}{l}\text { Fl. Oct-Jan } \\
\text { Fr. U }\end{array}$ & M & GKR1864 \\
\hline D. triflorum (L.) DC. & Kulaliya & $\begin{array}{l}\mathrm{D}, \mathrm{G}, \\
\mathrm{M}, \mathrm{T}\end{array}$ & $\mathrm{H}$ & $\begin{array}{l}\text { Ah, Fp, } \\
\text { Rs }\end{array}$ & $\mathrm{C}$ & $\begin{array}{l}\text { Fl. Jul-Dec } \\
\text { Fr. U }\end{array}$ & W & GKR1059 \\
\hline Erythrina fusca Lour. & Palita mandar & $\mathrm{M}, \mathrm{T}$ & $\mathrm{T}$ & Ah, Rs & $\mathrm{O}$ & $\begin{array}{l}\text { Fl. Feb-Apr } \\
\text { Fr. U }\end{array}$ & W & GKR1840 \\
\hline E. orientalis Murray & Mandar/Parijat & $\begin{array}{l}\mathrm{D}, \mathrm{G}, \\
\mathrm{M}, \mathrm{T}\end{array}$ & $\mathrm{T}$ & $\begin{array}{l}\text { Ah, Fp, } \\
\text { Rs }\end{array}$ & $\mathrm{C}$ & $\begin{array}{l}\text { Fl. Jul-Nov } \\
\text { Fr. U }\end{array}$ & W & GKR0953 \\
\hline
\end{tabular}

(Table contd.) 


\begin{tabular}{|c|c|c|c|c|c|c|c|c|}
\hline Scientific Name & Bangla name & District & Habit & Habitat & Occ. & Fl./Fr. Time & Use & RSE \\
\hline E. stricta Roxb. & Mandar & $\mathrm{D}, \mathrm{G}$ & $\mathrm{T}$ & $\mathrm{Ah}, \mathrm{Fp}$ & $\mathrm{O}$ & $\begin{array}{l}\text { Fl. Jan-May } \\
\text { Fr. U }\end{array}$ & $\mathrm{W}$ & GKR1317 \\
\hline Lablab purpureus (L.) Sweet & Shim & G & $\mathrm{H}$ & $\mathrm{Fp}$ & $\mathrm{O}$ & $\begin{array}{l}\text { Fl. Jul-Sep } \\
\text { Fr. U }\end{array}$ & $\mathrm{V}$ & GKR1992 \\
\hline Lathyrus aphaca $\mathrm{L}$. & Kasari & M & $\mathrm{H}$ & Rs & $\mathrm{O}$ & $\begin{array}{l}\text { Fl. Feb-Apr } \\
\text { Fr. U }\end{array}$ & $\mathrm{C}$ & GKR0220 \\
\hline $\begin{array}{l}\text { Leucaena leucocephala } \\
\text { (Lam.) de Wit }\end{array}$ & Ipil-Ipil & $\begin{array}{l}\mathrm{D}, \mathrm{G}, \\
\mathrm{M}, \mathrm{T}\end{array}$ & $\mathrm{T}$ & $\mathrm{Fp}, \mathrm{Rs}$ & $\mathrm{C}$ & $\begin{array}{l}\text { Fl. Apr-Jul } \\
\text { Fr. U }\end{array}$ & Fw & GKR1396 \\
\hline Medicago lupulina $\mathrm{L}$. & & $\mathrm{T}$ & $\mathrm{H}$ & $\mathrm{Ah}, \mathrm{Fp}$ & $\mathrm{O}$ & $\begin{array}{l}\text { Fl. Apr-Aug } \\
\text { Fr. U }\end{array}$ & W & GKR1633 \\
\hline $\begin{array}{l}\text { Mucuna bracteata DC. ex } \\
\text { Kurz }\end{array}$ & Unknown & $\mathrm{D}$ & $\mathrm{S}$ & Rs & $\mathrm{E}$ & $\begin{array}{l}\text { Fl. Jan-Apr } \\
\text { Fr. U }\end{array}$ & $\mathrm{W}$ & GKR1270 \\
\hline M. pruriens (L.) DC. & Alkushi & G & $\mathrm{H}$ & $\mathrm{Fp}$ & $\mathrm{O}$ & $\begin{array}{l}\text { Fl. Sep-Oct } \\
\text { Fr. Oct-Nov }\end{array}$ & M & GKR1997 \\
\hline $\begin{array}{l}\text { Pithecellobium dulce } \\
\text { (Roxb.) Benth. }\end{array}$ & Gilapi fal & $\mathrm{D}, \mathrm{M}$ & $\mathrm{T}$ & Ah, Rs & $\mathrm{O}$ & $\begin{array}{l}\text { Fl. Dec-Feb } \\
\text { Fr. U }\end{array}$ & $\begin{array}{l}\mathrm{Fd}, \\
\mathrm{Fw}\end{array}$ & GKR1386 \\
\hline $\begin{array}{l}\text { Pterocarpus macrocarpus } \\
\text { Kurz }\end{array}$ & Gumbury & $\mathrm{D}, \mathrm{G}$ & $\mathrm{T}$ & Rs & $\mathrm{O}$ & $\begin{array}{l}\text { Fl. Feb-Apr } \\
\text { Fr. U }\end{array}$ & $\mathrm{T}$ & GKR1703 \\
\hline P. santalinus L.f. & Raktachandan & M & $\mathrm{T}$ & $\mathrm{Ah}$ & $\mathrm{CE}$ & $\begin{array}{l}\text { Fl. Feb-Apr } \\
\text { Fr. U }\end{array}$ & $\begin{array}{l}\mathrm{D}, \\
\mathrm{M}, \\
\mathrm{O}\end{array}$ & GKR2510 \\
\hline $\begin{array}{l}\text { Senna obtusifolia }(\mathrm{L} .) \text { Irwin } \\
\& \text { Barneby }\end{array}$ & $\begin{array}{l}\text { Chakunda, Gol } \\
\text { Eski }\end{array}$ & $\mathrm{M}, \mathrm{T}$ & $\mathrm{H}$ & Rs & $\mathrm{O}$ & $\begin{array}{l}\text { Fl. Jul-Dec } \\
\text { Fr. U }\end{array}$ & M & GKR2101 \\
\hline $\begin{array}{l}\text { Sesbania cannabina (Retz.) } \\
\text { Poir. }\end{array}$ & Dhainchi & $\mathrm{D}$ & $\mathrm{H}$ & $\mathrm{Ah}$ & $\mathrm{O}$ & $\begin{array}{l}\text { Fl. Jul-Dec } \\
\text { Fr. U }\end{array}$ & $\mathrm{W}$ & GKR1282 \\
\hline S. sesban (L.) Merr. & Dhancha & $\mathrm{D}$ & $\mathrm{S}$ & $\mathrm{Fp}$ & $\mathrm{O}$ & Fl. \& Fr. Sep-Dec & Fo & GKR1327 \\
\hline Tamarindus indica $\mathrm{L}$. & Tentul & $\begin{array}{l}\mathrm{D}, \mathrm{G}, \\
\mathrm{M}\end{array}$ & $\mathrm{T}$ & $\begin{array}{l}\mathrm{Bb}, \mathrm{Fp}, \\
\mathrm{Rs}\end{array}$ & $\mathrm{O}$ & $\begin{array}{l}\text { Fl. Apr-Jun } \\
\text { Fr. U }\end{array}$ & $\begin{array}{l}\text { Fr, } \\
\mathrm{T}\end{array}$ & GKR1610 \\
\hline $\begin{array}{l}\text { Tephrosia purpurea (L.) } \\
\text { Pers. }\end{array}$ & Jangli neel & $\mathrm{D}$ & $\mathrm{H}$ & $\mathrm{Fp}$ & $\mathrm{O}$ & $\begin{array}{l}\text { Fl. Oct-Dec } \\
\text { Fr. U }\end{array}$ & $\mathrm{W}$ & GKR1670 \\
\hline Uraria lagopus DC. & $\begin{array}{l}\text { Chakuley, } \\
\text { Chakulia }\end{array}$ & G & $\mathrm{H}$ & Rs & $\mathrm{O}$ & $\begin{array}{l}\text { Fl. Sep-Nov } \\
\text { Fr. U }\end{array}$ & $\mathrm{W}$ & GKR0260 \\
\hline Vigna mungo (L.) Hepper & Maskolai & $\mathrm{D}, \mathrm{M}$ & $\mathrm{H}$ & $\mathrm{Ah}, \mathrm{Fp}$ & $\mathrm{O}$ & $\begin{array}{l}\text { Fl. Nov-Dec } \\
\text { Fr. U }\end{array}$ & $\mathrm{C}$ & GKR1163 \\
\hline \multicolumn{9}{|l|}{ LYTHRACEAE } \\
\hline Ammannia multiflora Roxb. & $\begin{array}{l}\text { Many-flower } \\
\text { ammannia }\end{array}$ & $\mathrm{T}$ & $\mathrm{H}$ & $\mathrm{Fp}, \mathrm{Rs}$ & $\mathrm{O}$ & Fl. \& Fr. Sep-Dec & $\mathrm{W}$ & GKR2161 \\
\hline A. baccifera $\mathrm{L}$. & Banmarich & M & $\mathrm{H}$ & Rs & $\mathrm{O}$ & $\begin{array}{l}\text { Fl. Sep-Dec } \\
\text { Fr. U }\end{array}$ & W & GKR0214 \\
\hline Lagerstroemia indica $\mathrm{L}$. & Chhotojarul & $\begin{array}{l}\mathrm{G}, \mathrm{M}, \\
\mathrm{T}\end{array}$ & $\mathrm{T}$ & $\mathrm{Fp}, \mathrm{Rs}$ & $\mathrm{O}$ & $\begin{array}{l}\text { Fl. Jun-Sep } \\
\text { Fr. U }\end{array}$ & $\mathrm{T}$ & GKR2288 \\
\hline L. parviflora Roxb. & Tilajarul & $\mathrm{G}, \mathrm{T}$ & $\mathrm{T}$ & Rs & $\mathrm{O}$ & $\begin{array}{l}\text { Fl. Feb-May } \\
\text { Fr. U }\end{array}$ & $\mathrm{T}$ & GKR2869 \\
\hline L. speciosa (L.) Pers. & Pannyajarul & G & $\mathrm{T}$ & Rs & $\mathrm{O}$ & $\begin{array}{l}\text { Fl. Jun-Aug } \\
\text { Fr. U }\end{array}$ & $\mathrm{T}$ & GKR0281 \\
\hline Lawsonia inermis L. & Mehedi & $\begin{array}{l}\mathrm{D}, \mathrm{G}, \\
\mathrm{M}, \mathrm{T}\end{array}$ & $\mathrm{T}$ & $\mathrm{Ah}$ & $\mathrm{C}$ & $\begin{array}{l}\text { Fl. Jan-Apr } \\
\text { Fr. U }\end{array}$ & $\mathrm{D}$ & GKR0311 \\
\hline $\begin{array}{l}\text { Rotala rotundifolia (Buch.- } \\
\text { Ham. ex Roxb.) Koehne }\end{array}$ & Unknown & M & $\mathrm{H}$ & Rs & $\mathrm{O}$ & Fl. \& Fr. Sep-Mar & W & GKR0156 \\
\hline
\end{tabular}

(Table contd.) 


\begin{tabular}{|c|c|c|c|c|c|c|c|c|}
\hline Scientific Name & Bangla name & District & Habit & Habitat & Occ. & Fl./Fr. Time & Use & RSE \\
\hline \multicolumn{9}{|l|}{ MYRTACEAE } \\
\hline $\begin{array}{l}\text { Eucalyptus camaldulensis } \\
\text { Dehnhardt }\end{array}$ & Eucalyptus & $\begin{array}{l}\mathrm{D}, \mathrm{G} \\
\mathrm{M}, \mathrm{T}\end{array}$ & $\mathrm{T}$ & Fp, Rs & $\mathrm{C}$ & $\begin{array}{l}\text { Fl. Jul-Feb } \\
\text { Fr. U }\end{array}$ & $\mathrm{T}$ & GKR1167 \\
\hline Psidium guajava $\mathrm{L}$. & Peyara & $\begin{array}{l}\mathrm{D}, \mathrm{G}, \\
\mathrm{M}\end{array}$ & $\mathrm{T}$ & $\mathrm{Ah}$ & $\mathrm{O}$ & $\begin{array}{l}\text { Fl. Mar-May } \\
\text { Fr. U }\end{array}$ & $\begin{array}{l}\mathrm{Fr} \\
\mathrm{M}\end{array}$ & GKR0030 \\
\hline Syzygium cumini (L.) Skeels & Jam & $\begin{array}{l}\mathrm{D}, \mathrm{G}, \\
\mathrm{M}, \mathrm{T}\end{array}$ & $\mathrm{T}$ & $\mathrm{Ah}$ & $\mathrm{C}$ & $\begin{array}{l}\text { Fl. Mar-May } \\
\text { Fr. U }\end{array}$ & $\begin{array}{l}\text { Fr, } \\
\mathrm{T}\end{array}$ & GKR3248 \\
\hline S. fruticosum DC. & Putijam & $\begin{array}{l}\mathrm{D}, \mathrm{G} \\
\mathrm{M}, \mathrm{T}\end{array}$ & $\mathrm{T}$ & $\mathrm{Fp}, \mathrm{Rs}$ & $\mathrm{C}$ & $\begin{array}{l}\text { Fl. Mar-Apr } \\
\text { Fr. U }\end{array}$ & $\mathrm{W}$ & GKR4668 \\
\hline $\begin{array}{l}\text { S. samarangense (Blume) } \\
\text { Merr. \& L.M.Perry }\end{array}$ & Jamrul & $\begin{array}{l}\mathrm{D}, \mathrm{G} \\
\mathrm{M}, \mathrm{T}\end{array}$ & $\mathrm{T}$ & $\mathrm{Fp}$ & $\mathrm{C}$ & $\begin{array}{l}\text { Fl. Feb-Mar } \\
\text { Fr. U }\end{array}$ & $\mathrm{Fr}$ & GKR2108 \\
\hline S. jambos (L.) Alston & Golapjam & $\begin{array}{l}\mathrm{D}, \mathrm{G} \\
\mathrm{M}\end{array}$ & $\mathrm{T}$ & $\mathrm{Ah}, \mathrm{Fp}$ & $\mathrm{O}$ & $\begin{array}{l}\text { Fl. Feb-Apr } \\
\text { Fr. U }\end{array}$ & $\mathrm{Fr}$ & GKR1940 \\
\hline \multicolumn{9}{|l|}{ PUNICACEAE } \\
\hline Punica granatum $\mathrm{L}$. & Anar & $\begin{array}{l}\mathrm{D}, \mathrm{G}, \\
\mathrm{M}, \mathrm{T}\end{array}$ & $\mathrm{ST}$ & Ah, Hy & $\mathrm{C}$ & $\begin{array}{l}\text { Fl. May-Jul } \\
\text { Fr. U }\end{array}$ & Fr & GKR3094 \\
\hline \multicolumn{9}{|l|}{ ONAGRACEAE } \\
\hline $\begin{array}{l}\text { Ludwigia adscendens (L.) } \\
\text { Hara }\end{array}$ & Keshordam & G & $\mathrm{H}$ & $\mathrm{Ah}, \mathrm{Fp}$ & $\mathrm{O}$ & $\begin{array}{l}\text { Fl.\& Fr. Through- } \\
\text { out the year }\end{array}$ & We & GKR0837 \\
\hline $\begin{array}{l}\text { L. hyssopifolia (G. Don) } \\
\text { Exell }\end{array}$ & Panimarich & $\begin{array}{l}\mathrm{D}, \mathrm{G} \\
\mathrm{M}, \mathrm{T}\end{array}$ & $\mathrm{H}$ & $\begin{array}{l}\text { Ah, Fp, } \\
\text { Rs }\end{array}$ & $\mathrm{C}$ & $\begin{array}{l}\text { Fl. Dec-Jan } \\
\text { Fr. U }\end{array}$ & $\begin{array}{l}\text { Aq } \\
\text { we }\end{array}$ & GKR0524 \\
\hline L. perennis $\mathrm{L}$. & Banlong & $\mathrm{M}, \mathrm{T}$ & $\mathrm{H}$ & $\mathrm{Fp}, \mathrm{Rs}$ & $\mathrm{O}$ & $\begin{array}{l}\text { Fl. \& Fr. Through- } \\
\text { out the year }\end{array}$ & We & GKR0086 \\
\hline \multicolumn{9}{|l|}{ COMBRETACEAE } \\
\hline $\begin{array}{l}\text { Quisqualis indica (Thunb.) } \\
\text { Gagnep. }\end{array}$ & Madhobilata & $\mathrm{D}, \mathrm{T}$ & $S$ & $\mathrm{Fp}, \mathrm{Rs}$ & $\mathrm{O}$ & $\begin{array}{l}\text { Fl. Mar-Nov } \\
\text { Fr. U }\end{array}$ & Or & GKR1542 \\
\hline $\begin{array}{l}\text { Terminalia arjuna (Roxb. Ex } \\
\text { DC.) Wight \& Arn. }\end{array}$ & Arjun & M & $\mathrm{T}$ & Rs & $\mathrm{O}$ & $\begin{array}{l}\text { Fl. Apr-Jul } \\
\text { Fr. U }\end{array}$ & M & GKR1861 \\
\hline T. bellirica (Gaertn.) Roxb. & Bohera & $\mathrm{D}, \mathrm{G}$ & $\mathrm{T}$ & Rs & $\mathrm{O}$ & $\begin{array}{l}\text { Fl. Apr-May } \\
\text { Fr. U }\end{array}$ & M & GKR2006 \\
\hline T. chebula Retz. & Haritaki & $\begin{array}{l}\mathrm{D}, \mathrm{G} \\
\mathrm{M}, \mathrm{T}\end{array}$ & $\mathrm{T}$ & $\begin{array}{l}\text { Ah, Fp, } \\
\text { Rs }\end{array}$ & $\mathrm{C}$ & $\begin{array}{l}\text { Fl. Mar-May } \\
\text { Fr. U }\end{array}$ & M & GKR0330 \\
\hline T. catappa $\mathrm{L}$. & Kat badam & $\begin{array}{l}\mathrm{D}, \mathrm{M}, \\
\mathrm{T}\end{array}$ & $\mathrm{T}$ & $\mathrm{Fp}, \mathrm{Rs}$ & $\mathrm{O}$ & $\begin{array}{l}\text { Fl. Mar-Jun } \\
\text { Fr. Jul-Sep }\end{array}$ & $\begin{array}{l}\mathrm{M}, \\
\mathrm{T}\end{array}$ & GKR0664 \\
\hline $\begin{array}{l}\text { CORNACEAE } \\
\text { Alangium salviifolium (L.f.) } \\
\text { Wangerin }\end{array}$ & Aikha & $\begin{array}{l}\mathrm{D}, \mathrm{G} \\
\mathrm{M}, \mathrm{T}\end{array}$ & $\mathrm{T}$ & $\mathrm{Bb}, \mathrm{Fp}$ & $\mathrm{C}$ & $\begin{array}{l}\text { Fl. Mar-Aug } \\
\text { Fr. U }\end{array}$ & $\mathrm{FW}$ & GKR1153 \\
\hline $\begin{array}{l}\text { OLACACEAE } \\
\text { Olax acuminata Wall, ex } \\
\text { Benth. }\end{array}$ & Unknown & $\mathrm{M}, \mathrm{T}$ & ST & Rs & $\mathrm{O}$ & $\begin{array}{l}\text { Fl. Mar-Sep } \\
\text { Fr. U }\end{array}$ & M & GKR0282 \\
\hline \multicolumn{9}{|l|}{ LORANTHACEAE } \\
\hline $\begin{array}{l}\text { Dendrophthoe falcata (L. f.) } \\
\text { Etting. }\end{array}$ & $\begin{array}{l}\text { Maandaa, } \\
\text { Bandha, } \\
\text { Pharulla }\end{array}$ & $\mathrm{T}$ & $S$ & Rs & $\mathrm{O}$ & $\begin{array}{l}\text { Fl. Jan-Feb } \\
\text { Fr. U }\end{array}$ & M & GKR2221 \\
\hline $\begin{array}{l}\text { Helixanthera cylindrica } \\
\text { (Jack ex Roxb.) Danser }\end{array}$ & Unknown & $\mathrm{D}$ & $\mathrm{H}$ & Fp, Rs & $\mathrm{O}$ & $\begin{array}{l}\text { Fl. Jun-Aug } \\
\text { Fr. U }\end{array}$ & $\mathrm{W}$ & GKR1606 \\
\hline $\begin{array}{l}\text { PUTRANJIVACEAE } \\
\text { Putranjiva roxburghii Wall. }\end{array}$ & Putranjiva & M & $\mathrm{T}$ & Rs & $\mathrm{O}$ & $\begin{array}{l}\text { Fl. Mar-Aug } \\
\text { Fr. U }\end{array}$ & M & GKR1238 \\
\hline
\end{tabular}

(Table contd.) 


\begin{tabular}{|c|c|c|c|c|c|c|c|c|}
\hline Scientific Name & Bangla name & District & Habit & Habitat & Occ. & Fl./Fr. Time & Use & RSE \\
\hline \multicolumn{9}{|l|}{ EUPHORBIACEAE } \\
\hline Acalypha ciliata Forssk. & Muktajhuri & $\begin{array}{l}\mathrm{D}, \mathrm{M}, \\
\mathrm{T}\end{array}$ & $\mathrm{H}$ & $\begin{array}{l}\text { Ah, Fp, } \\
\text { Rs }\end{array}$ & $\mathrm{O}$ & $\begin{array}{l}\text { Fl. Nov-Aug } \\
\text { Fr. U }\end{array}$ & M & GKR1337 \\
\hline A. indica $\mathrm{L}$. & Muktajhuri & $\begin{array}{l}\mathrm{D}, \mathrm{G} \\
\mathrm{M}, \mathrm{T}\end{array}$ & $\mathrm{H}$ & $\begin{array}{l}\text { Ah, Fp, } \\
\text { Rs }\end{array}$ & $\mathrm{C}$ & $\begin{array}{l}\text { Fl. Mar-Oct } \\
\text { Fr. U }\end{array}$ & M & GKR0467 \\
\hline $\begin{array}{l}\text { Baliospermum montanum } \\
\text { (Willd.) Muell.-Arg. }\end{array}$ & Dandi, Danti & $\mathrm{D}$ & $\mathrm{S}$ & Fp, Rs & $\mathrm{O}$ & $\begin{array}{l}\text { Fl. Aug-Mar } \\
\text { Fr. U }\end{array}$ & M & GKR0510 \\
\hline Bridelia tomentosa Blume & Khoi, Serai & $\mathrm{G}, \mathrm{T}$ & $\mathrm{S}$ & Rs & $\mathrm{O}$ & $\begin{array}{l}\text { Fl. \& Fr. Through- } \\
\text { out the year }\end{array}$ & $\mathrm{W}$ & GKR0250 \\
\hline $\begin{array}{l}\text { Chrozophora rottleri } \\
\text { (Geiseler) A. Juss. ex } \\
\text { Spreng. }\end{array}$ & Khudi Ojra & $\mathrm{D}, \mathrm{G}$ & $\mathrm{H}$ & Rs & $\mathrm{O}$ & $\begin{array}{l}\text { Fl. Feb-Aug } \\
\text { Fr. U }\end{array}$ & M & GKR0394 \\
\hline $\begin{array}{l}\text { C. plicata (Vahl) A.Juss. ex } \\
\text { Spreng. }\end{array}$ & $\begin{array}{l}\text { Karu, Kadu, } \\
\text { Chireta }\end{array}$ & $\mathrm{D}, \mathrm{M}$ & $\mathrm{s}$ & $\mathrm{Fp}$, Rs & $\mathrm{O}$ & $\begin{array}{l}\text { Fl. Feb-Sep } \\
\text { Fr. U }\end{array}$ & M & GKR0040 \\
\hline $\begin{array}{l}\text { Codiaeum variegatum (L.) } \\
\text { Rumph. ex A.Juss. }\end{array}$ & Pata bahar & $\begin{array}{l}\mathrm{D}, \mathrm{G}, \\
\mathrm{M}, \mathrm{T}\end{array}$ & $\mathrm{T}$ & $\mathrm{Ah}, \mathrm{Fp}$ & $\mathrm{C}$ & $\begin{array}{l}\text { Fl. Mar-Jun } \\
\text { Fr. U }\end{array}$ & Or & GKR0149 \\
\hline $\begin{array}{l}\text { Croton bonplandianum } \\
\text { Baill. }\end{array}$ & Banmarich & $\begin{array}{l}\mathrm{D}, \mathrm{G} \\
\mathrm{M}, \mathrm{T}\end{array}$ & $\mathrm{H}$ & Fp, Rs & $\mathrm{C}$ & $\begin{array}{l}\text { Fl. Nov-May } \\
\text { Fr. U }\end{array}$ & M & GKR0478 \\
\hline C. roxburghii Balakr. & $\begin{array}{l}\text { Baragachh, } \\
\text { Mashimud, } \\
\text { Putla }\end{array}$ & $\mathrm{G}, \mathrm{T}$ & $\mathrm{H}$ & Rs & $\mathrm{O}$ & $\begin{array}{l}\text { Fl. \& Fr. Through- } \\
\text { out the year }\end{array}$ & M & GKR0339 \\
\hline C. caudatus Geiseler & $\begin{array}{l}\text { Climbing } \\
\text { Croton }\end{array}$ & $\mathrm{T}$ & $\mathrm{S}$ & Rs & $\mathrm{O}$ & $\begin{array}{l}\text { Fl. Mar-May } \\
\text { Fr. U }\end{array}$ & M & GKR0140 \\
\hline Euphorbia cotinifolia $\mathrm{L}$. & Red Spurge & $\mathrm{T}$ & $\mathrm{S}$ & Rs & $\mathrm{O}$ & $\begin{array}{l}\text { Fl. Mar-Jun } \\
\text { Fr. Nov-Jan }\end{array}$ & Or & GKR2190 \\
\hline E. heterophylla $\mathrm{L}$. & milkweed & $\mathrm{D}$ & $\mathrm{H}$ & Fp & $\mathrm{O}$ & $\begin{array}{l}\text { Fl.\& Fr. Through- } \\
\text { out the year }\end{array}$ & $\mathrm{W}$ & GKR0493 \\
\hline E. hirta $\mathrm{L}$. & Dudhia & $\begin{array}{l}\mathrm{D}, \mathrm{G} \\
\mathrm{M}, \mathrm{T}\end{array}$ & $\mathrm{H}$ & $\begin{array}{l}\text { Ah, Fp, } \\
\text { Rs }\end{array}$ & $\mathrm{C}$ & $\begin{array}{l}\text { Fl. \& Fr. Through- } \\
\text { out the year }\end{array}$ & M & GKR0486 \\
\hline E. miliii Des Moul. & Kontok Mukut & G & $\mathrm{S}$ & Rs & $\mathrm{O}$ & $\begin{array}{l}\text { Fl. Apr-Aug } \\
\text { Fr. U }\end{array}$ & M & GKR0509 \\
\hline E. prostrata Ait. & red euphorbia & $\mathrm{D}, \mathrm{G}$ & $\mathrm{H}$ & $\begin{array}{l}\text { Ah, Fp, } \\
\text { Rs }\end{array}$ & $\mathrm{O}$ & $\begin{array}{l}\text { Fl. Mar-Jun } \\
\text { Fr. U }\end{array}$ & M & GKR0416 \\
\hline E. thymifolia $\mathrm{L}$. & $\begin{array}{l}\text { Swetkarni, } \\
\text { Swetkan }\end{array}$ & $\begin{array}{l}\mathrm{D}, \mathrm{G}, \\
\mathrm{M}, \mathrm{T}\end{array}$ & $\mathrm{H}$ & $\begin{array}{l}\text { Ah, Fp, } \\
\text { Rs }\end{array}$ & $\mathrm{C}$ & $\begin{array}{l}\text { Fl. Nov-May } \\
\text { Fr. U }\end{array}$ & We & GKR0482 \\
\hline E. tirucalli $\mathrm{L}$. & Lankasij & G & $\mathrm{S}$ & Rs & $\mathrm{O}$ & $\begin{array}{l}\text { Fl. May-Jul } \\
\text { Fr. U }\end{array}$ & M & GKR0798 \\
\hline $\begin{array}{l}\text { Excoecaria bicolor (Hassk.) } \\
\text { Zoll. ex Hassk. }\end{array}$ & Laily Majnu & M & $S$ & Rs & $\mathrm{O}$ & $\begin{array}{l}\text { Fl. Jan-Apr } \\
\text { Fr. U }\end{array}$ & Or & GKR0005 \\
\hline Flueggea microcarpa Blume & $\begin{array}{l}\text { Shikori, Sitka, } \\
\text { Sitki }\end{array}$ & M & $S$ & Rs & $\mathrm{O}$ & $\begin{array}{l}\text { Fl. Mar-Sep } \\
\text { Fr. U }\end{array}$ & $\mathrm{W}$ & GKR0082 \\
\hline $\begin{array}{l}\text { Glochidion assamicum (Müll } \\
\text {. Arg.) Hook. f. }\end{array}$ & $\begin{array}{l}\text { Poniatori, } \\
\text { Dostomi }\end{array}$ & M & S & Fp, Rs & $\mathrm{O}$ & $\begin{array}{l}\text { Fl. Nov-Feb } \\
\text { Fr. U }\end{array}$ & M & GKR0136 \\
\hline $\begin{array}{l}\text { Hevea brasiliensis (Willd. ex } \\
\text { A.Juss.) Müll.Arg. }\end{array}$ & Rubber & $\begin{array}{l}\mathrm{D}, \mathrm{G}, \\
\mathrm{M}, \mathrm{T}\end{array}$ & $\mathrm{T}$ & $\begin{array}{l}\mathrm{Bb}, \mathrm{Fp} \\
\mathrm{Rs}\end{array}$ & $\mathrm{C}$ & $\begin{array}{l}\text { Fl. May-Aug } \\
\text { Fr. U }\end{array}$ & $\mathrm{R}$ & GKR1628 \\
\hline Jatropha curcas L. & Baghbherenda & $\mathrm{D}$ & S & Rs & $\mathrm{O}$ & $\begin{array}{l}\text { Fl. Apr-Jul } \\
\text { Fr. U }\end{array}$ & $\begin{array}{l}\mathrm{M}, \\
\mathrm{O}\end{array}$ & GKR1626 \\
\hline J. gossypifolia L. & Lalbherenda & $\mathrm{D}$ & S & Rs & $\mathrm{O}$ & $\begin{array}{l}\text { Fl. Jul-Sep } \\
\text { Fr. U }\end{array}$ & M & GKR1653 \\
\hline
\end{tabular}

(Table contd.) 


\begin{tabular}{|c|c|c|c|c|c|c|c|c|}
\hline Scientific Name & Bangla name & District & Habit & Habitat & Occ. & Fl./Fr. Time & Use & RSE \\
\hline J. panduraefolia Andri & Joyti & $\mathrm{M}$ & $S$ & Fp, Rs & $\mathrm{O}$ & $\begin{array}{l}\text { Fl. \& Fr. Through- } \\
\text { out the year }\end{array}$ & Or & GKR2089 \\
\hline $\begin{array}{l}\text { Macaranga denticulata } \\
\text { (Blume) Müll.Arg. }\end{array}$ & Bura, Ratabura & $\mathrm{M}, \mathrm{T}$ & $\mathrm{T}$ & Fp, Rs & $\mathrm{O}$ & $\begin{array}{l}\text { Fl. Apr-Jun } \\
\text { Fr. May-Oct }\end{array}$ & $\begin{array}{l}\text { Fw, } \\
\mathrm{W}\end{array}$ & GKR1668 \\
\hline M. indica Wight & Chakkulla & $\mathrm{D}, \mathrm{G}$ & $\mathrm{T}$ & Fp & $\mathrm{O}$ & $\begin{array}{l}\text { Fl. Oct-Dec } \\
\text { Fr. U }\end{array}$ & $\begin{array}{l}\text { Fw, } \\
\mathrm{W}\end{array}$ & GKR0571 \\
\hline M. peltata (Roxb.) Müll.Arg. & $\begin{array}{l}\text { Chakkula, } \\
\text { Burna }\end{array}$ & $\begin{array}{l}\mathrm{D}, \mathrm{G}, \\
\mathrm{M}\end{array}$ & $\mathrm{T}$ & Fp, Rs & $\mathrm{O}$ & $\begin{array}{l}\text { Fl. Jan-Feb } \\
\text { Fr. U }\end{array}$ & $\begin{array}{l}\text { Fw, } \\
\mathrm{T}\end{array}$ & GKR0352 \\
\hline $\begin{array}{l}\text { Mallotus philippensis (Lam.) } \\
\text { Müll.-Arg. }\end{array}$ & $\begin{array}{l}\text { Sindur, } \\
\text { Kingul,Kamala }\end{array}$ & $\mathrm{T}$ & $\mathrm{T}$ & Rs & $\mathrm{O}$ & $\begin{array}{l}\text { Fl. Jan-Mar } \\
\text { Fr. U }\end{array}$ & M & GKR0295 \\
\hline $\begin{array}{l}\text { M. repandus (Willd.) Muell.- } \\
\text { Arg. }\end{array}$ & Bon notoy & $\mathrm{G}, \mathrm{T}$ & $\mathrm{H}$ & $\mathrm{Ah}, \mathrm{Fp}$ & $\mathrm{O}$ & $\begin{array}{l}\text { Fl. Mar-May } \\
\text { Fr. U }\end{array}$ & M & GKR0290 \\
\hline $\begin{array}{l}\text { Pedilanthus tithymaloides } \\
\text { (L.) Poit. }\end{array}$ & Rangchita & $\mathrm{M}, \mathrm{T}$ & $\mathrm{H}$ & $\mathrm{Fp}$ & $\mathrm{O}$ & $\begin{array}{l}\text { Fl. Jun-Aug } \\
\text { Fr. U }\end{array}$ & Or & GKR0918 \\
\hline Phyllanthus niruri L. & Bhui amla & $\mathrm{D}, \mathrm{T}$ & $S$ & Rs & $\mathrm{O}$ & $\begin{array}{l}\text { Fl. Jul-Oct } \\
\text { Fr. U }\end{array}$ & M & GKR1015 \\
\hline Ricinus communis $\mathrm{L}$. & Venna, Reri & $\begin{array}{l}\mathrm{D}, \mathrm{G}, \\
\mathrm{M}, \mathrm{T}\end{array}$ & ST & $\begin{array}{l}\text { Ah, Fp, } \\
\text { Rs }\end{array}$ & $\mathrm{C}$ & $\begin{array}{l}\text { Fl. May-Jun } \\
\text { Fr. U }\end{array}$ & $\mathrm{O}$ & GKR0475 \\
\hline $\begin{array}{l}\text { Suregada multiflora (A. } \\
\text { Juss.) Bail. }\end{array}$ & Ghotlatkon & G & $\mathrm{T}$ & Rs & $\mathrm{O}$ & $\begin{array}{l}\text { Fl. Mar-May } \\
\text { Fr. U }\end{array}$ & Fw & GKR2026 \\
\hline Trewia nudiflora L. & Latim & $\begin{array}{l}\mathrm{D}, \mathrm{G}, \\
\mathrm{M}, \mathrm{T}\end{array}$ & $\mathrm{T}$ & Rs & $\mathrm{C}$ & $\begin{array}{l}\text { Fl. \& Fr. May- } \\
\text { Nov }\end{array}$ & W & GKR0123 \\
\hline \multicolumn{9}{|l|}{ PHYLLANTHACEAE } \\
\hline $\begin{array}{l}\text { Antidesma ghaesembilla } \\
\text { Gaertn. }\end{array}$ & $\begin{array}{l}\text { Black Currant } \\
\text { Tree }\end{array}$ & M & $\mathrm{S}$ & Rs & $\mathrm{O}$ & $\begin{array}{l}\text { Fl. Mar-Sep } \\
\text { Fr. Jun-Dec }\end{array}$ & Fr & GKR2075 \\
\hline A. acidum Retz. & $\begin{array}{l}\text { Mutta,Mathag } \\
\text { ach }\end{array}$ & M & $S$ & Rs & $\mathrm{O}$ & $\begin{array}{l}\text { Fl. Mar-Oct } \\
\text { Fr. Jun-Jan }\end{array}$ & W & GKR0219 \\
\hline $\begin{array}{l}\text { Baccaurea motleyana } \\
\text { (Müll.Arg.) Müll.Arg. }\end{array}$ & Lotkon,Bhubhi & $\begin{array}{l}\mathrm{D}, \mathrm{G}, \\
\mathrm{M}, \mathrm{T}\end{array}$ & $\mathrm{T}$ & $\begin{array}{l}\mathrm{Ah}, \\
\mathrm{Bb}, \mathrm{Fp}\end{array}$ & $\mathrm{C}$ & $\begin{array}{l}\text { Fl. Mar-Oct } \\
\text { Fr. U }\end{array}$ & Fr & GKR0789 \\
\hline Emblica officinalis Gaertn. & Amlaki & M & $\mathrm{T}$ & $\mathrm{Ah}$ & $\mathrm{O}$ & $\begin{array}{l}\text { Fl. Feb-May } \\
\text { Fr. Jun-Sep }\end{array}$ & M & GKR1918 \\
\hline $\begin{array}{l}\text { Phyllanthus acidus (L.) } \\
\text { Skeels }\end{array}$ & Orboroi & $\begin{array}{l}\mathrm{D}, \mathrm{G}, \\
\mathrm{M}, \mathrm{T}\end{array}$ & $\mathrm{T}$ & Ah, Hy & $\mathrm{C}$ & $\begin{array}{l}\text { Fl. Jan-Jun } \\
\text { Fr. Jun-Aug }\end{array}$ & $\begin{array}{l}\mathrm{Ch} \\
\mathrm{M}\end{array}$ & GKR0065 \\
\hline P. reticulatus Poir. & $\begin{array}{l}\text { Chitki, } \\
\text { Panseuli }\end{array}$ & $\begin{array}{l}\mathrm{D}, \mathrm{G} \\
\mathrm{M}, \mathrm{T}\end{array}$ & $S$ & $\begin{array}{l}\text { Ah, Fp, } \\
\text { Rs }\end{array}$ & $\mathrm{C}$ & $\begin{array}{l}\text { Fl. Jul-Sep } \\
\text { Fr. U }\end{array}$ & W & GKR0087 \\
\hline P. urinaria $\mathrm{L}$. & Hazarmani & $\begin{array}{l}\mathrm{G}, \mathrm{M}, \\
\mathrm{T}\end{array}$ & $\mathrm{H}$ & Fp, Rs & $\mathrm{C}$ & $\begin{array}{l}\text { Fl. Jul-Oct } \\
\text { Fr. U }\end{array}$ & M & GKR1968 \\
\hline \multicolumn{9}{|l|}{ RHAMNACEAE } \\
\hline Ziziphus mauritiana Lamk. & Kul, Boroi & $\mathrm{D}, \mathrm{T}$ & $\mathrm{T}$ & Rs & $\mathrm{O}$ & $\begin{array}{l}\text { Fl. Jul-Nov } \\
\text { Fr. U }\end{array}$ & Fr & GKR1611 \\
\hline Z. rugosa Lam. & Bon boroi & $\mathrm{T}$ & ST & Rs & $\mathrm{O}$ & $\begin{array}{l}\text { Fl. Jan-Apr } \\
\text { Fr. U }\end{array}$ & W & GKR0283 \\
\hline \multicolumn{9}{|l|}{ VITACEAE } \\
\hline $\begin{array}{l}\text { Ampelocissus barbata } \\
\text { (Wall.) Planch. }\end{array}$ & Jarila-lahari & M & $\mathrm{H}$ & Rs & $\mathrm{O}$ & $\begin{array}{l}\text { Fl. Mar-Sep } \\
\text { Fr. U }\end{array}$ & M & GKR0138 \\
\hline A. latifolia (Roxb.) Planch. & $\begin{array}{l}\text { Gowalia Lata, } \\
\text { Pani-lata }\end{array}$ & $\mathrm{G}, \mathrm{M}$ & $\mathrm{H}$ & $\mathrm{Ah}$ & $\mathrm{O}$ & $\begin{array}{l}\text { Fl. May-Jun, } \\
\text { Fr. Oct }\end{array}$ & $\mathrm{M}$ & GKR0047 \\
\hline $\begin{array}{l}\text { Cayratia japonica (Thunb.) } \\
\text { Gagnep. }\end{array}$ & Bushkiller & $\mathrm{D}, \mathrm{T}$ & $\mathrm{H}$ & $\mathrm{Ah}, \mathrm{Fp}$ & $\mathrm{O}$ & $\begin{array}{l}\text { Fl. Mar-Jun } \\
\text { Fr. U }\end{array}$ & M & GKR1540 \\
\hline
\end{tabular}

(Table contd.) 


\begin{tabular}{|c|c|c|c|c|c|c|c|c|}
\hline Scientific Name & Bangla name & District & Habit & Habitat & Occ. & Fl./Fr. Time & Use & RSE \\
\hline C. trifolia (L.) Domin & Amal-lata & $\begin{array}{l}\mathrm{D}, \mathrm{G}, \\
\mathrm{M}, \mathrm{T}\end{array}$ & $\mathrm{S}$ & $\begin{array}{l}\text { Ah, Fp, } \\
\text { Rs }\end{array}$ & $\mathrm{C}$ & $\begin{array}{l}\text { Fl. \& Fr. Through- } \\
\text { out the year }\end{array}$ & $\mathrm{M}$ & GKR0100 \\
\hline Cissus adnata Roxb. & $\begin{array}{l}\text { Alianga lata, } \\
\text { Bhatia-lota. }\end{array}$ & G & $\mathrm{S}$ & $\mathrm{Fp}$ & $\mathrm{O}$ & $\begin{array}{l}\text { Fl. Jun-Jul. } \\
\text { Fr. Aug-Sep }\end{array}$ & M & GKR0889 \\
\hline $\begin{array}{l}\text { Leea acuminata Wallich ex } \\
\text { Clarke }\end{array}$ & Kukur-jihwa & $\mathrm{D}$ & $\mathrm{S}$ & $\mathrm{Fp}$ & $\mathrm{O}$ & $\begin{array}{l}\text { Fl. Aug-Oct } \\
\text { Fr. U }\end{array}$ & $\mathrm{W}$ & GKR1692 \\
\hline $\begin{array}{l}\text { L. macrophylla Roxb. ex } \\
\text { Hornem. }\end{array}$ & $\begin{array}{l}\text { Dholsamudra, } \\
\text { Hathikani }\end{array}$ & $\mathrm{D}$ & $\mathrm{S}$ & Rs & $\mathrm{O}$ & $\begin{array}{l}\text { Fl. Jul-Sep } \\
\text { Fr. U }\end{array}$ & M & GKR1259 \\
\hline L. robusta Roxb. & Unknown & $\mathrm{D}, \mathrm{T}$ & $\mathrm{S}$ & $\mathrm{Ah}$ & $\mathrm{O}$ & $\begin{array}{l}\text { Fl. Jul-Dec } \\
\text { Fr. U }\end{array}$ & $\mathrm{W}$ & GKR1961 \\
\hline $\begin{array}{l}\text { Tetrastigma bracteolatum } \\
\text { (Wall.) Planch. }\end{array}$ & Golgoti lota & $\mathrm{D}$ & $\mathrm{S}$ & $\mathrm{Fp}$ & $\mathrm{O}$ & Fl. \& Fr. Aug-Oct & $\mathrm{W}$ & GKR1683 \\
\hline $\begin{array}{l}\text { T. leucostaphylum (Dennst.) } \\
\text { Alston }\end{array}$ & $\begin{array}{l}\text { Indian } \\
\text { Chestnut Vine }\end{array}$ & $\mathrm{D}$ & $\mathrm{S}$ & $\mathrm{Fp}$ & $\mathrm{O}$ & $\begin{array}{l}\text { Fl. Mar-Aug } \\
\text { Fr. U }\end{array}$ & $\mathrm{W}$ & GKR1680 \\
\hline Vitis vinifera $\mathrm{L}$. & Angur & M & $\mathrm{H}$ & $\mathrm{Ah}$ & $\mathrm{O}$ & $\begin{array}{l}\text { Fl. May-Jul } \\
\text { Fr. U }\end{array}$ & $\mathrm{Fr}$ & GKR0121 \\
\hline \multicolumn{9}{|l|}{ LINACEAE } \\
\hline Linum usitatissimum L. & Tisi & M & $\mathrm{H}$ & Rs & $\mathrm{O}$ & $\begin{array}{l}\text { Fl. Jun-Aug, } \\
\text { Fr. Jul-Oct }\end{array}$ & $\mathrm{O}$ & GKR0049 \\
\hline \multicolumn{9}{|l|}{ SAPINDACEAE } \\
\hline $\begin{array}{l}\text { Cardiospermum } \\
\text { halicacabum } \mathrm{L} .\end{array}$ & $\begin{array}{l}\text { Latapatkari, } \\
\text { Kapalphutki }\end{array}$ & $\mathrm{D}$ & $\mathrm{H}$ & Rs & $\mathrm{O}$ & $\begin{array}{l}\text { Fl. Jul-Feb } \\
\text { Fr. U }\end{array}$ & M & GKR1349 \\
\hline Dimocarpus longan Lour. & Kath litchi & $\mathrm{D}, \mathrm{G}$ & $\mathrm{T}$ & $\mathrm{Ah}$ & $\mathrm{O}$ & $\begin{array}{l}\text { Fl. Apr-Aug } \\
\text { Fr. U }\end{array}$ & $\mathrm{Fr}$ & GKR0354 \\
\hline $\begin{array}{l}\text { Lepisanthes rubiginosa } \\
\text { (Roxb.) Leenh. }\end{array}$ & $\begin{array}{l}\text { Bon lichu, } \\
\text { KakJam/ } \\
\text { Horina }\end{array}$ & $\mathrm{G}, \mathrm{T}$ & $\mathrm{T}$ & Rs & $\mathrm{O}$ & $\begin{array}{l}\text { Fl. Mar-May } \\
\text { Fr. U }\end{array}$ & M & GKR0281 \\
\hline $\begin{array}{l}\text { L. senegalensis (Poir.) } \\
\text { Leenh. }\end{array}$ & Gotahorina & $\mathrm{D}, \mathrm{G}$ & $\mathrm{T}$ & Ah, Rs & $\mathrm{O}$ & $\begin{array}{l}\text { Fl. Nov-Jun } \\
\text { Fr. U }\end{array}$ & M & GKR0728 \\
\hline Litchi sinensis Sonner & Lichu & $\begin{array}{l}\mathrm{D}, \mathrm{G}, \\
\mathrm{M}\end{array}$ & $\mathrm{T}$ & $\mathrm{Ah}, \mathrm{Fp}$ & $\mathrm{O}$ & $\begin{array}{l}\text { Fl. Jan-Mar } \\
\text { Fr. U }\end{array}$ & $\mathrm{Fr}$ & GKR1103 \\
\hline \multicolumn{9}{|l|}{ BURSERACEAE } \\
\hline Garuga pinnata Roxb. & Kapila, Jum & $\mathrm{D}$ & $\mathrm{T}$ & Rs & $\mathrm{O}$ & $\begin{array}{l}\text { Fl. Feb-May } \\
\text { Fr. U }\end{array}$ & $\mathrm{W}$ & GKR2604 \\
\hline \multicolumn{9}{|l|}{ ANACARDIACEAE } \\
\hline $\begin{array}{l}\text { Lannea coromandelica } \\
\text { (Houtt.) Merr. }\end{array}$ & Jiga, Jika, Jeol & $\mathrm{D}, \mathrm{G}$ & $\mathrm{T}$ & Fp, Rs & $\mathrm{O}$ & Fl. \& Fr. Jan-May & $\begin{array}{l}\mathrm{Re}, \\
\mathrm{T}\end{array}$ & GKR2997 \\
\hline Mangifera indica $\mathrm{L}$. & Aam & $\begin{array}{l}\mathrm{D}, \mathrm{G}, \\
\mathrm{M}\end{array}$ & $\mathrm{T}$ & $\mathrm{Fp}, \mathrm{Rs}$ & $\mathrm{O}$ & $\begin{array}{l}\text { Fl. Jan-Mar } \\
\text { Fr. May-Jun }\end{array}$ & $\begin{array}{l}\mathrm{Fr}, \\
\mathrm{T}\end{array}$ & GKR0079 \\
\hline Spondias mombin L. & Amra & $\begin{array}{l}\mathrm{D}, \mathrm{G}, \\
\mathrm{M}\end{array}$ & $\mathrm{T}$ & $\mathrm{Ah}, \mathrm{Fp}$ & $\mathrm{O}$ & $\begin{array}{l}\text { Fl. Jan-May } \\
\text { Fr. U }\end{array}$ & $\begin{array}{l}\mathrm{Fr} \\
\mathrm{M}\end{array}$ & GKR0239 \\
\hline \multicolumn{9}{|l|}{ MELIACEAE } \\
\hline $\begin{array}{l}\text { Aphanamixis polystachya } \\
\text { (Wall.) R.Parker }\end{array}$ & Roina, Pitraj & $\begin{array}{l}\mathrm{D}, \mathrm{G}, \\
\mathrm{M}, \mathrm{T}\end{array}$ & $\mathrm{T}$ & Ah, Rs & $\mathrm{C}$ & $\begin{array}{l}\text { Fl. Oct-Feb } \\
\text { Fr. U }\end{array}$ & $\mathrm{O}, \mathrm{T}$ & GKR0328 \\
\hline Azadirachta indica A. Juss. & Neem & $\mathrm{D}, \mathrm{G}$ & $\mathrm{T}$ & Rs & $\mathrm{O}$ & $\begin{array}{l}\text { Fl. Feb-Apr } \\
\text { Fr. U }\end{array}$ & M & GKR0412 \\
\hline Cedrela odorata $\mathrm{L}$. & Cedar & $\mathrm{D}$ & $\mathrm{T}$ & $\mathrm{Ah}, \mathrm{Rs}$ & $\mathrm{O}$ & $\begin{array}{l}\text { Fl. Jan-Jun } \\
\text { Fr. U }\end{array}$ & $\mathrm{T}$ & GKR2339 \\
\hline Dysoxylum excelsum blume. & Dingori & $\mathrm{T}$ & $\mathrm{T}$ & Rs & $\mathrm{O}$ & $\begin{array}{l}\text { Fl. Dec-Jul, } \\
\text { Fr. Jun-Jul }\end{array}$ & $\mathrm{T}$ & GKR0958 \\
\hline
\end{tabular}

(Table contd.) 


\begin{tabular}{|c|c|c|c|c|c|c|c|c|}
\hline Scientific Name & Bangla name & District & Habit & Habitat & Occ. & Fl./Fr. Time & Use & RSE \\
\hline $\begin{array}{l}\text { Khaya anthotheca (Welw.) } \\
\text { C.DC. }\end{array}$ & Lambu & $\begin{array}{l}\mathrm{D}, \mathrm{G}, \\
\mathrm{M}\end{array}$ & $\mathrm{T}$ & $\mathrm{Fp}, \mathrm{Rs}$ & $\mathrm{O}$ & $\begin{array}{l}\text { Fl. Sep-Dec } \\
\text { Fr. U }\end{array}$ & $\mathrm{T}$ & GKR1021 \\
\hline Swietenia mahogani $\mathrm{L}$. & Mehagoni & $\begin{array}{l}\mathrm{D}, \mathrm{G}, \\
\mathrm{M}\end{array}$ & $\mathrm{T}$ & Fp & $\mathrm{O}$ & $\begin{array}{l}\text { Fl. Apr-May } \\
\text { Fr. U }\end{array}$ & $\mathrm{T}$ & GKR0924 \\
\hline Toona ciliata M.Roem. & Tun & M & $\mathrm{T}$ & Rs & $\mathrm{O}$ & Fl. \& Fr. Feb-May & W & GKR0032 \\
\hline \multicolumn{9}{|l|}{ RUTACEAE } \\
\hline Aegle marmelos (L.) Corr. & Bel & $\begin{array}{l}\mathrm{D}, \mathrm{G} \\
\mathrm{M}, \mathrm{T}\end{array}$ & $\mathrm{T}$ & $\begin{array}{l}\text { Ah, Fp, } \\
\text { Rs }\end{array}$ & $\mathrm{C}$ & $\begin{array}{l}\text { Fl. Mar-Jun } \\
\text { Fr. U }\end{array}$ & $\begin{array}{l}\text { Fr, } \\
\mathrm{M}\end{array}$ & GKR1372 \\
\hline Citrus limon (L.) Osbeck. & $\begin{array}{l}\text { Goralebu, } \\
\text { Baralebu }\end{array}$ & $\begin{array}{l}\mathrm{D}, \mathrm{G}, \\
\mathrm{M}, \mathrm{T}\end{array}$ & ST & $\mathrm{Ah}, \mathrm{Fp}$ & $\mathrm{C}$ & $\begin{array}{l}\text { Fl. \& Fr. Through- } \\
\text { out the year }\end{array}$ & $\begin{array}{l}\mathrm{M} \\
\mathrm{O}\end{array}$ & GKR1572 \\
\hline C. grandis (L.) Osbeck & Jambura & $\begin{array}{l}\mathrm{D}, \mathrm{G}, \\
\mathrm{M}\end{array}$ & $\mathrm{T}$ & Ah, Hy & $\mathrm{O}$ & $\begin{array}{l}\text { Fl. Feb-Apr } \\
\text { Fr. U }\end{array}$ & $\begin{array}{l}\text { Fr, } \\
\mathrm{M}\end{array}$ & GKR1743 \\
\hline C. reticulata Blanco & Kamla & $\mathrm{T}$ & ST & $\mathrm{Fp}$ & $\mathrm{O}$ & $\begin{array}{l}\text { Fl. Sep-Nov } \\
\text { Fr. U }\end{array}$ & $\begin{array}{l}\text { Fr, } \\
\mathrm{M}\end{array}$ & GKR1342 \\
\hline Feronia elephantum Corrêa & Kodbel & $\begin{array}{l}\mathrm{D}, \mathrm{G} \\
\mathrm{M}, \mathrm{T}\end{array}$ & $\mathrm{T}$ & Fp, Rs & $\mathrm{C}$ & $\begin{array}{l}\text { Fl. Feb-Mar } \\
\text { Fr. U }\end{array}$ & $\begin{array}{l}\text { Fr, } \\
\text { M }\end{array}$ & GKR2962 \\
\hline $\begin{array}{l}\text { Glycosmis pentaphylla } \\
\text { (Retz.) DC. }\end{array}$ & $\begin{array}{l}\text { Motkila, } \\
\text { Asheora }\end{array}$ & $\begin{array}{l}\mathrm{D}, \mathrm{G} \\
\mathrm{M}, \mathrm{T}\end{array}$ & $\mathrm{S}$ & $\begin{array}{l}\text { Ah, Fp, } \\
\text { Rs }\end{array}$ & $\mathrm{C}$ & $\begin{array}{l}\text { Fl. Feb-Apr } \\
\text { Fr. U }\end{array}$ & $\begin{array}{l}\text { Fw, } \\
\mathrm{M}\end{array}$ & GKR0054 \\
\hline $\begin{array}{l}\text { Murraya koenigii (L.) } \\
\text { Spreng. }\end{array}$ & Curry pata & M & $\mathrm{S}$ & Ah, Rs & $\mathrm{O}$ & $\begin{array}{l}\text { Fl. Mar-May } \\
\text { Fr. U }\end{array}$ & M & GKR1866 \\
\hline M. paniculata (L.) Jack & Kamini & $\mathrm{M}, \mathrm{T}$ & ST & $\mathrm{Ah}, \mathrm{Rs}$ & $\mathrm{O}$ & $\begin{array}{l}\text { Fl. Mar-May } \\
\text { Fr. U }\end{array}$ & $\mathrm{Pi}$ & GKR2382 \\
\hline Zanthoxylum rhetsa DC. & Bajna & G & $\mathrm{T}$ & Rs & $\mathrm{O}$ & $\begin{array}{l}\text { Fl. Mar-Nov } \\
\text { Fr. U }\end{array}$ & $\mathrm{O}$ & GKR0338 \\
\hline \multicolumn{9}{|l|}{ OXALIDACEAE } \\
\hline Averrhoa carambola $\mathrm{L}$. & Kamranga & $\mathrm{M}, \mathrm{T}$ & $\mathrm{T}$ & $\mathrm{Ah}$ & $\mathrm{O}$ & $\begin{array}{l}\text { Fl. \& Fr. Through- } \\
\text { out the year }\end{array}$ & Fr & GKR2535 \\
\hline Oxalis corniculata $\mathrm{L}$. & Amrul & $\mathrm{D}, \mathrm{G}, \mathrm{T}$ & $\mathrm{H}$ & $\begin{array}{l}\text { Ah, Fp, } \\
\text { Rs }\end{array}$ & $\mathrm{O}$ & $\begin{array}{l}\text { Fl. Jun-Aug } \\
\text { Fr. U }\end{array}$ & We & GKR0445 \\
\hline \multicolumn{9}{|l|}{ APIACEAE } \\
\hline Centella asiatica (L.) Urban & Thankuni & $\begin{array}{l}\mathrm{D}, \mathrm{G} \\
\mathrm{M}, \mathrm{T}\end{array}$ & $\mathrm{H}$ & $\begin{array}{l}\text { Ah, Fp, } \\
\text { Rs }\end{array}$ & $\mathrm{C}$ & $\begin{array}{l}\text { Fl. Apr-May } \\
\text { Fr. U }\end{array}$ & M & GKR0018 \\
\hline Coriandrum sativum $\mathrm{L}$. & Dhonia & M & $\mathrm{H}$ & Rs & $\mathrm{O}$ & $\begin{array}{l}\text { Fl. Dec-Apr } \\
\text { Fr. U }\end{array}$ & $\mathrm{S}$ & GKR0182 \\
\hline Eryngium foetidum $\mathrm{L}$. & Bilatidhania & $\begin{array}{l}\mathrm{D}, \mathrm{M}, \\
\mathrm{T}\end{array}$ & $\mathrm{H}$ & Fp, Rs & $\mathrm{O}$ & $\begin{array}{l}\text { Fl. Apr-May } \\
\text { Fr. U }\end{array}$ & $\mathrm{S}$ & GKR0729 \\
\hline Oenanthe crocata $\mathrm{L}$. & $\begin{array}{l}\text { Water- } \\
\text { hemlock }\end{array}$ & $\mathrm{D}, \mathrm{T}$ & $\mathrm{H}$ & $\mathrm{Fp}$ & $\mathrm{O}$ & $\begin{array}{l}\text { Fl. Jun-Sep } \\
\text { Fr. U }\end{array}$ & $\mathrm{P}$ & GKR3404 \\
\hline \multicolumn{9}{|l|}{ APOCYNACEAE } \\
\hline Alstonia scholaris (L.) R. Br. & Chhatim & $\begin{array}{l}\mathrm{D}, \mathrm{G} \\
\mathrm{M}, \mathrm{T}\end{array}$ & $\mathrm{T}$ & $\begin{array}{l}\text { Ep, Fp, } \\
\text { Rs }\end{array}$ & $\mathrm{C}$ & $\begin{array}{l}\text { Fl. Oct-Feb } \\
\text { Fr. U }\end{array}$ & $\begin{array}{l}\text { Fw, } \\
\mathrm{T}\end{array}$ & GKR0754 \\
\hline Carissa carandas $\mathrm{L}$. & Koromcha & $\mathrm{D}, \mathrm{T}$ & $\mathrm{S}$ & $\mathrm{Ah}$ & $\mathrm{O}$ & $\begin{array}{l}\text { Fl. Jan-Jun } \\
\text { Fr. U }\end{array}$ & Fr & GKR0320 \\
\hline $\begin{array}{l}\text { Gymnema inodorum (Lour.) } \\
\text { Decne. }\end{array}$ & Unknown & $\mathrm{D}$ & $\mathrm{S}$ & $\mathrm{Fp}$ & $\mathrm{O}$ & $\begin{array}{l}\text { Fl. Jun-Aug } \\
\text { Fr. U }\end{array}$ & M & GKR0141 \\
\hline $\begin{array}{l}\text { Holarrhena antidysenterica } \\
\text { (L.) Wall }\end{array}$ & Kurchi & $\mathrm{D}, \mathrm{G}$ & $\mathrm{S}$ & Rs & $\mathrm{O}$ & $\begin{array}{l}\text { Fl. Apr-Oct } \\
\text { Fr. U }\end{array}$ & M & GKR0353 \\
\hline $\begin{array}{l}\text { Ichnocarpus frutescens (L.) } \\
\text { W.T.Aiton }\end{array}$ & Dudhilata & $\mathrm{D}, \mathrm{M}$ & $\mathrm{S}$ & $\mathrm{Fp}, \mathrm{Rs}$ & $\mathrm{O}$ & $\begin{array}{l}\text { Fl. Aug-Dec } \\
\text { Fr. U }\end{array}$ & M & GKR0058 \\
\hline
\end{tabular}

(Table contd.) 


\begin{tabular}{|c|c|c|c|c|c|c|c|c|}
\hline Scientific Name & Bangla name & District & Habit & Habitat & Occ. & Fl./Fr. Time & Use & RSE \\
\hline Nerium oleander L. & Korobi & $\mathrm{G}, \mathrm{T}$ & $S$ & Hy, Rs & $\mathrm{O}$ & $\begin{array}{l}\text { Fl. Apr-Sep } \\
\text { Fr. U }\end{array}$ & $\mathrm{M}$ & GKR1223 \\
\hline Plumeria obtusa $\mathrm{L}$. & Kathgolop & $\mathrm{T}$ & ST & Rs & $\mathrm{O}$ & $\begin{array}{l}\text { Fl. \& Fr. Through- } \\
\text { out the year }\end{array}$ & Or & GKR1078 \\
\hline $\begin{array}{l}\text { Rauvolfia serpentina (L.) } \\
\text { Benth. ex Kurz }\end{array}$ & Sarpagandha & $\mathrm{D}, \mathrm{M}$ & $\mathrm{H}$ & Ah, Rs & $\mathrm{E}$ & $\begin{array}{l}\text { Fl. Apr-Oct } \\
\text { Fr. U }\end{array}$ & M & GKR1277 \\
\hline $\begin{array}{l}\text { Tabernaemontana coronaria } \\
\text { (Jacq.) Willd. }\end{array}$ & Tagar & $\begin{array}{l}\mathrm{G}, \mathrm{M}, \\
\mathrm{T}\end{array}$ & S & Fp, Rs & $\mathrm{O}$ & $\begin{array}{l}\text { Fl. Apr-Sep } \\
\text { Fr. U }\end{array}$ & Or & GKR1984 \\
\hline T. dichotoma Roxb. ex Wall. & Kath Maloti & $\mathrm{D}, \mathrm{M}$ & ST & $\mathrm{Ah}$ & $\mathrm{O}$ & $\begin{array}{l}\text { Fl. Apr-Jul } \\
\text { Fr. U }\end{array}$ & Or & GKR2077 \\
\hline $\begin{array}{l}\text { Thevetia peruviana (Pers.) } \\
\text { K. Schum. }\end{array}$ & $\begin{array}{l}\text { Kalkephul, } \\
\text { Halde Korobi }\end{array}$ & $\mathrm{T}$ & ST & Rs & $\mathrm{O}$ & $\begin{array}{l}\text { Fl. \& Fr. Through- } \\
\text { out the year }\end{array}$ & Or & GKR2223 \\
\hline \multicolumn{9}{|l|}{ ASCLEPIADACEAE } \\
\hline $\begin{array}{l}\text { Calotropis procera (Aiton) } \\
\text { Dryand. }\end{array}$ & Akonda & $\begin{array}{l}\mathrm{D}, \mathrm{G}, \\
\mathrm{M}, \mathrm{T}\end{array}$ & S & Ah, Rs & $\mathrm{C}$ & $\begin{array}{l}\text { Fl. Nov-Feb } \\
\text { Fr. U }\end{array}$ & M & GKR0109 \\
\hline $\begin{array}{l}\text { Gongronema nepalense } \\
\text { (Wall.) Decne. }\end{array}$ & Unknown & $\mathrm{T}$ & S & Fp, Rs & $\mathrm{O}$ & $\begin{array}{l}\text { Fl. Jun-Jul } \\
\text { Fr. U }\end{array}$ & $\mathrm{W}$ & GKR0278 \\
\hline $\begin{array}{l}\text { Marsdenia tenacissima } \\
\text { (Roxb.) Moon }\end{array}$ & $\begin{array}{l}\text { Chitti, Jitti, } \\
\text { Siti }\end{array}$ & G & $S$ & Rs & $\mathrm{O}$ & $\begin{array}{l}\text { Fl. Aug-Dec } \\
\text { Fr. U }\end{array}$ & M & GKR1987 \\
\hline \multicolumn{9}{|l|}{ SOLANACEAE } \\
\hline Capsicum frutescens $\mathrm{L}$. & Morich & $\mathrm{M}, \mathrm{T}$ & $\mathrm{H}$ & $\mathrm{Ah}, \mathrm{Fp}$ & $\mathrm{O}$ & $\begin{array}{l}\text { Fl. \& Fr. Through- } \\
\text { out the year }\end{array}$ & $\mathrm{S}$ & GKR1905 \\
\hline Datura metel L. & Kalo Datura & $\begin{array}{l}\mathrm{D}, \mathrm{M}, \\
\mathrm{T}\end{array}$ & S & Fp, Rs & $\mathrm{O}$ & $\begin{array}{l}\text { Fl. Mar-Dec } \\
\text { Fr. U }\end{array}$ & M & GKR0223 \\
\hline D. stramonium $\mathrm{L}$. & Datura & $\begin{array}{l}\mathrm{D}, \mathrm{M}, \\
\mathrm{T}\end{array}$ & $S$ & $\begin{array}{l}\text { Ah, Fp, } \\
\text { Rs }\end{array}$ & $\mathrm{O}$ & $\begin{array}{l}\text { Fl. Jul-Sep } \\
\text { Fr. U }\end{array}$ & M & GKR0490 \\
\hline $\begin{array}{l}\text { Nicotiana plumbaginifolia } \\
\text { Viv. }\end{array}$ & Bantamak & $\begin{array}{l}\mathrm{D}, \mathrm{G}, \\
\mathrm{M}, \mathrm{T}\end{array}$ & $\mathrm{H}$ & $\begin{array}{l}\text { Ah, Fp, } \\
\text { Rs }\end{array}$ & $\mathrm{C}$ & $\begin{array}{l}\text { Fl. Mar-Aug } \\
\text { Fr. U }\end{array}$ & We & GKR0002 \\
\hline Physalis angulata $\mathrm{L}$. & Unknown & $\mathrm{M}, \mathrm{T}$ & $\mathrm{H}$ & Fp, Rs & $\mathrm{O}$ & $\begin{array}{l}\text { Fl. Jul-Dec } \\
\text { Fr. U }\end{array}$ & M & GKR0980 \\
\hline P. minima $\mathrm{L}$. & $\begin{array}{l}\text { Tepari/Kapalp } \\
\text { hutki }\end{array}$ & $\begin{array}{l}\mathrm{D}, \mathrm{G}, \\
\mathrm{M}, \mathrm{T}\end{array}$ & $\mathrm{H}$ & $\begin{array}{l}\text { Ah, Fp, } \\
\text { Rs }\end{array}$ & $\mathrm{C}$ & $\begin{array}{l}\text { Fl. Nov-Feb } \\
\text { Fr. Jul-Oct }\end{array}$ & M & GKR0458 \\
\hline Solanum capsicoides All. & Tita Begun & G & $S$ & $\mathrm{Ah}$ & $\mathrm{O}$ & $\begin{array}{l}\text { Fl. Jun-Aug } \\
\text { Fr. U }\end{array}$ & $\mathrm{V}$ & GKR0255 \\
\hline S. melongena $\mathrm{L}$. & Begun & $\mathrm{D}$ & $\mathrm{H}$ & Rs & $\mathrm{O}$ & $\begin{array}{l}\text { Fl. Apr-Dec } \\
\text { Fr. U }\end{array}$ & $\mathrm{V}$ & GKR1287 \\
\hline S. nigrum L. & Titbegun & $\begin{array}{l}\mathrm{D}, \mathrm{G}, \\
\mathrm{M}, \mathrm{T}\end{array}$ & $\mathrm{H}$ & $\begin{array}{l}\text { Ah, Fp, } \\
\text { Rs }\end{array}$ & $\mathrm{C}$ & $\begin{array}{l}\text { Fl. Dec-Mar } \\
\text { Fr. U }\end{array}$ & $\mathrm{W}$ & GKR0435 \\
\hline S. sisymbrifolium Lamk. & Kanta Begun & G & $\mathrm{H}$ & $\mathrm{Ah}$ & $\mathrm{O}$ & $\begin{array}{l}\text { Fl. May-Aug } \\
\text { Fr. U }\end{array}$ & $\mathrm{W}$ & GKR0384 \\
\hline S. surattense Burm. F. & Kantikari & $\mathrm{D}$ & $\mathrm{H}$ & $\mathrm{Fp}$ & $\mathrm{O}$ & $\begin{array}{l}\text { Fl. Dec-Mar, } \\
\text { Fr. Jul-Sep }\end{array}$ & M & GKR0646 \\
\hline S. torvum $\mathrm{Sw}$. & Tit Begun & $\begin{array}{l}\mathrm{D}, \mathrm{M}, \\
\mathrm{T}\end{array}$ & $\mathrm{H}$ & $\begin{array}{l}\text { Ah, Fp, } \\
\text { Rs }\end{array}$ & $\mathrm{O}$ & $\begin{array}{l}\text { Fl. Feb-Apr, } \\
\text { Fr. Aug-Nov }\end{array}$ & $\mathrm{W}$ & GKR1265 \\
\hline S. indicum $\mathrm{L}$. & Phutki Begun & $\mathrm{D}$ & $\mathrm{H}$ & Fp, Rs & $\mathrm{O}$ & $\begin{array}{l}\text { Fl. Feb-Apr, } \\
\text { Fr. Aug-Nov }\end{array}$ & M & GKR1663 \\
\hline $\begin{array}{l}\text { S. xanthocarpum Schrad. \& } \\
\text { H. Wendl. }\end{array}$ & Kantikari & $\begin{array}{l}\mathrm{D}, \mathrm{G}, \\
\mathrm{M}\end{array}$ & $\mathrm{H}$ & Ep, Rs & $\mathrm{O}$ & $\begin{array}{l}\text { Fl. Jun-Aug } \\
\text { Fr. U }\end{array}$ & M & GKR0479 \\
\hline
\end{tabular}

(Table contd.) 


\begin{tabular}{|c|c|c|c|c|c|c|c|c|}
\hline Scientific Name & Bangla name & District & Habit & Habitat & Occ. & Fl./Fr. Time & Use & RSE \\
\hline \multicolumn{9}{|l|}{ CONVOLVULACEAE } \\
\hline $\begin{array}{l}\text { Aniseia martinicensis (Jacq.) } \\
\text { Choisy }\end{array}$ & Shadamati & M & $\mathrm{H}$ & $\mathrm{Fp}$ & $\mathrm{CE}$ & $\begin{array}{l}\text { Fl. Sep-Nov } \\
\text { Fr. U }\end{array}$ & $\mathrm{W}$ & GKR2578 \\
\hline $\begin{array}{l}\text { Argyreia capitiformis (Poir.) } \\
\text { Ooststr. }\end{array}$ & Unknown & $\mathrm{G}, \mathrm{M}$ & $\mathrm{S}$ & $\mathrm{Ah}, \mathrm{Fp}$ & $\mathrm{O}$ & $\begin{array}{l}\text { Fl. Sep-Dec } \\
\text { Fr. U }\end{array}$ & M & GKR0365 \\
\hline Cuscuta chinensis Lam. & $\begin{array}{l}\text { China } \\
\text { swarnalata }\end{array}$ & M & $\mathrm{H}$ & Fp, Rs & $\mathrm{O}$ & $\begin{array}{l}\text { Fl. Nov-Mar } \\
\text { Fr. U }\end{array}$ & M & GKR0198 \\
\hline Cuscuta reflexa Roxb. & Swarnalata & $\mathrm{G}, \mathrm{M}$ & $\mathrm{H}$ & Fp, Rs & $\mathrm{O}$ & Fl. \& Fr. Dec-Feb & M & GKR2533 \\
\hline Evolvulus nummularius (L.) & $\begin{array}{l}\text { Bhuikamri, } \\
\text { Bhuiokra }\end{array}$ & $\begin{array}{l}\mathrm{D}, \mathrm{G} \\
\mathrm{M}, \mathrm{T}\end{array}$ & $\mathrm{H}$ & $\begin{array}{l}\text { Ah, Fp, } \\
\text { Rs }\end{array}$ & $\mathrm{C}$ & $\begin{array}{l}\text { Fl. \& Fr. Through- } \\
\text { out the year }\end{array}$ & We & GKR0055 \\
\hline Ipomoea alba $\mathrm{L}$. & Moonflower & $\mathrm{M}, \mathrm{T}$ & $\mathrm{H}$ & Rs & $\mathrm{O}$ & $\begin{array}{l}\text { Fl. Jul-Oct } \\
\text { Fr. U }\end{array}$ & $\mathrm{W}$ & GKR0904 \\
\hline I. aquatica Forssk. & Panikalmi & $\begin{array}{l}\mathrm{D}, \mathrm{G} \\
\mathrm{M}, \mathrm{T}\end{array}$ & $\mathrm{H}$ & Ah, Rs & $\mathrm{C}$ & $\begin{array}{l}\text { Fl. Jun-Aug } \\
\text { Fr. U }\end{array}$ & $\mathrm{V}$ & GKR0189 \\
\hline I. batatas (L.) Lamk. & Misti Alu & $\mathrm{G}, \mathrm{M}$ & $\mathrm{H}$ & $\mathrm{Fp}, \mathrm{Rs}$ & $\mathrm{O}$ & $\begin{array}{l}\text { Fl. May-Jul } \\
\text { Fr. U }\end{array}$ & $\mathrm{V}$ & GKR0211 \\
\hline I. mauritiana Jacq. & Bhumikumra & $\mathrm{D}, \mathrm{G}$ & $\mathrm{H}$ & $\mathrm{Ah}$ & $\mathrm{O}$ & $\begin{array}{l}\text { Fl. Aug-Sep } \\
\text { Fr. U }\end{array}$ & M & GKR1256 \\
\hline $\begin{array}{l}\text { Operculina turpethum (L.) } \\
\text { Silva Manso }\end{array}$ & Teorimul & $\mathrm{D}, \mathrm{M}$ & $\mathrm{H}$ & Ah, Rs & $\mathrm{O}$ & $\begin{array}{l}\text { Fl. Mar-Dec } \\
\text { Fr. U }\end{array}$ & M & GKR0576 \\
\hline $\begin{array}{l}\text { Merremia emarginata } \\
\text { (Burm. f.) Hallier f. }\end{array}$ & Indurkani & G & $\mathrm{H}$ & $\mathrm{Fp}, \mathrm{Rs}$ & $\mathrm{O}$ & Fl. \& Fr. Dec-Apr & M & GKR0791 \\
\hline $\begin{array}{l}\text { M. hederacea (Burm. f.) } \\
\text { Hallier f. }\end{array}$ & Sapussunda & $\mathrm{D}$ & $\mathrm{H}$ & Fp & $\mathrm{O}$ & Fl. Fr. Oct-Jan & M & GKR1635 \\
\hline $\begin{array}{l}\text { MENYANTHACEAE } \\
\text { Nymphoides indicum (L.) } \\
\text { Kuntze }\end{array}$ & Chandmala & M & $\mathrm{H}$ & Rs & $\mathrm{O}$ & $\begin{array}{l}\text { Fl. Jul-Sep } \\
\text { Fr. U }\end{array}$ & $\begin{array}{l}\text { Aq } \\
\text { we }\end{array}$ & GKR1169 \\
\hline \multicolumn{9}{|l|}{ BORAGINACEAE } \\
\hline Cordia dichotoma G.Forst. & Bowla & $\begin{array}{l}\mathrm{D}, \mathrm{G} \\
\mathrm{M}, \mathrm{T}\end{array}$ & $\mathrm{T}$ & $\begin{array}{l}\text { Ah, Ep, } \\
\text { Fp }\end{array}$ & $\mathrm{C}$ & $\begin{array}{l}\text { Fl. Mar - Apr, } \\
\text { Fr. Jul - Aug }\end{array}$ & $\begin{array}{l}\text { Fw, } \\
\mathrm{M}\end{array}$ & GKR0176 \\
\hline C. fragrantissima Kurz & Kaladuti & $\mathrm{T}$ & $\mathrm{T}$ & $\mathrm{Fp}$ & $\mathrm{O}$ & $\begin{array}{l}\text { Fl. Feb-Mar } \\
\text { Fr. U }\end{array}$ & $\mathrm{W}$ & GKR1966 \\
\hline Heliotropium indicum $\mathrm{L}$. & Hatisur & $\begin{array}{l}\mathrm{D}, \mathrm{G}, \\
\mathrm{M}, \mathrm{T}\end{array}$ & $\mathrm{H}$ & $\mathrm{Fp}, \mathrm{Rs}$ & $\mathrm{C}$ & $\begin{array}{l}\text { Fl. Sep-Mar } \\
\text { Fr. U }\end{array}$ & M & GKR0454 \\
\hline $\begin{array}{l}\text { Tournefortia roxburghii C. } \\
\text { B. Clarke }\end{array}$ & Shamshog & M & $\mathrm{S}$ & Rs & $\mathrm{CE}$ & $\begin{array}{l}\text { Fl. Feb-Nov } \\
\text { Fr. U }\end{array}$ & $\mathrm{W}$ & GKR1176 \\
\hline \multicolumn{9}{|l|}{ VERBENACEAE } \\
\hline Duranta repens $\mathrm{L}$. & $\begin{array}{l}\text { Golden } \\
\text { dewberry }\end{array}$ & G & $\mathrm{S}$ & $\mathrm{Ah}, \mathrm{Fp}$ & $\mathrm{O}$ & $\begin{array}{l}\text { Fl. \& Fr. Through- } \\
\text { out the year }\end{array}$ & M & GKR0345 \\
\hline $\begin{array}{l}\text { Lippia alba (Mill.) N.E.Br. } \\
\text { ex Britton \& P.Wilson }\end{array}$ & $\begin{array}{l}\text { Motmoti, } \\
\text { Motkhori }\end{array}$ & $\begin{array}{l}\mathrm{D}, \mathrm{G}, \\
\mathrm{M}\end{array}$ & $\mathrm{S}$ & $\begin{array}{l}\text { Ah, Fp, } \\
\text { Rs }\end{array}$ & $\mathrm{O}$ & $\begin{array}{l}\text { Fl. \& Fr. Through- } \\
\text { out the year }\end{array}$ & $\mathrm{W}$ & GKR0090 \\
\hline Phyla nodiflora (L.) Greene & Bhui Okar & $\begin{array}{l}\mathrm{D}, \mathrm{M} \\
\mathrm{T}\end{array}$ & $\mathrm{H}$ & $\begin{array}{l}\text { Ah, Fp, } \\
\text { Rs }\end{array}$ & $\mathrm{O}$ & $\begin{array}{l}\text { Fl. Nov-Dec } \\
\text { Fr. U }\end{array}$ & M & GKR0559 \\
\hline \multicolumn{9}{|l|}{ LAMIACEAE } \\
\hline Callicarpa arborea Roxb. & $\begin{array}{l}\text { Bormala, } \\
\text { Makanchi }\end{array}$ & $\mathrm{D}$ & $\mathrm{T}$ & $\mathrm{Fp}$ & $\mathrm{O}$ & $\begin{array}{l}\text { Fl. May-Oct, } \\
\text { Fr. Oct-Dec }\end{array}$ & M & GKR1694 \\
\hline $\begin{array}{l}\text { Clerodendrum indicum (L.) } \\
\text { Kuntze }\end{array}$ & Bamunhati & $\mathrm{D}, \mathrm{M}$ & $\mathrm{S}$ & $\mathrm{Fp}, \mathrm{Rs}$ & $\mathrm{O}$ & $\begin{array}{l}\text { Fl. Sep-Dec } \\
\text { Fr. U }\end{array}$ & M & GKR0465 \\
\hline C. infortunatum $\mathrm{L}$. & Bhant & $\mathrm{M}, \mathrm{T}$ & $\mathrm{S}$ & Rs & $\mathrm{O}$ & $\begin{array}{l}\text { Fl. Dec-Feb } \\
\text { Fr. U }\end{array}$ & M & GKR1828 \\
\hline
\end{tabular}

(Table contd.) 


\begin{tabular}{|c|c|c|c|c|c|c|c|c|}
\hline Scientific Name & Bangla name & District & Habit & Habitat & Occ. & Fl./Fr. Time & Use & RSE \\
\hline Gmelina arborea Roxb. & Gamar & $\begin{array}{l}\mathrm{D}, \mathrm{G}, \\
\mathrm{M}\end{array}$ & $\mathrm{T}$ & Fp, Rs & $\mathrm{O}$ & $\begin{array}{l}\text { Fl. Feb-Apr } \\
\text { Fr. May-Jun }\end{array}$ & $\begin{array}{l}\text { T, } \\
\text { Mi }\end{array}$ & GKR2977 \\
\hline Hyptis capitata Jacq. & Bilatitukma & M & $\mathrm{S}$ & $\mathrm{Fp}$ & $\mathrm{O}$ & $\begin{array}{l}\text { Fl.Sep-Jan } \\
\text { Fr. U }\end{array}$ & M & GKR0216 \\
\hline H. suaveolens (L.) Poit. & $\begin{array}{l}\text { Tukma, Bilatti } \\
\text { Tulas }\end{array}$ & $\mathrm{D}, \mathrm{M}$ & $\mathrm{H}$ & $\begin{array}{l}\text { Ah, Fp, } \\
\text { Rs }\end{array}$ & $\mathrm{O}$ & $\begin{array}{l}\text { Fl. Aug-Feb } \\
\text { Fr. U }\end{array}$ & M & GKR0488 \\
\hline Leonurus sibiricus $\mathrm{L}$. & Raktodrone & $\begin{array}{l}\mathrm{D}, \mathrm{G} \\
\mathrm{M}, \mathrm{T}\end{array}$ & $\mathrm{H}$ & $\begin{array}{l}\text { Ah, Fp, } \\
\text { Rs }\end{array}$ & $\mathrm{C}$ & $\begin{array}{l}\text { Fl. Jul-Sep } \\
\text { Fr. U }\end{array}$ & M & GKR0690 \\
\hline Leucas aspera (Willd.) Link. & Dandakalos & $\begin{array}{l}\mathrm{D}, \mathrm{G} \\
\mathrm{M}, \mathrm{T}\end{array}$ & $\mathrm{H}$ & $\begin{array}{l}\text { Ah, Fp, } \\
\text { Rs }\end{array}$ & $\mathrm{C}$ & $\begin{array}{l}\text { Fl. Nov-Feb } \\
\text { Fr. U }\end{array}$ & M & GKR0248 \\
\hline L. indica (L.) Sm. & Swetdron & $\mathrm{G}, \mathrm{T}$ & $\mathrm{H}$ & $\begin{array}{l}\text { Ah, Fp, } \\
\text { Rs }\end{array}$ & $\mathrm{O}$ & $\begin{array}{l}\text { Fl. Mar-Jul } \\
\text { Fr. U }\end{array}$ & M & GKR0407 \\
\hline L. vestita Benth. & Tita dron & $\mathrm{D}$ & $\mathrm{H}$ & $\mathrm{Fp}$ & $\mathrm{O}$ & $\begin{array}{l}\text { Fl. Nov-Feb } \\
\text { Fr. U }\end{array}$ & M & GKR1370 \\
\hline Mentha piperita $\mathrm{L}$. & $\begin{array}{l}\text { Pudina, } \\
\text { Mentha }\end{array}$ & $\begin{array}{l}\mathrm{D}, \mathrm{M}, \\
\mathrm{T}\end{array}$ & $\mathrm{H}$ & $\mathrm{Ah}$ & $\mathrm{O}$ & $\begin{array}{l}\text { Fl. Apr-Jan } \\
\text { Fr. U }\end{array}$ & M & GKR2393 \\
\hline Ocimum americanum $\mathrm{L}$. & Kalo-tulashi & $\mathrm{D}, \mathrm{G}, \mathrm{T}$ & $\mathrm{H}$ & $\mathrm{Ah}, \mathrm{Fp}$ & $\mathrm{O}$ & $\begin{array}{l}\text { Fl. \& Fr. Through- } \\
\text { out the year }\end{array}$ & M & GKR1614 \\
\hline O. gratissimum $\mathrm{L}$. & Ram tulsi & $\begin{array}{l}\mathrm{D}, \mathrm{M}, \\
\mathrm{T}\end{array}$ & $\mathrm{H}$ & $\mathrm{Ah}, \mathrm{Fp}$ & $\mathrm{O}$ & $\begin{array}{l}\text { Fl. Aug-Dec } \\
\text { Fr. U }\end{array}$ & M & GKR1260 \\
\hline O. sanctum $\mathrm{L}$. & Krishnatulsi & $\begin{array}{l}\mathrm{D}, \mathrm{M}, \\
\mathrm{T}\end{array}$ & $\mathrm{H}$ & $\mathrm{Ah}, \mathrm{Fp}$ & $\mathrm{O}$ & $\begin{array}{l}\text { Fl. Sep-Mar } \\
\text { Fr. U }\end{array}$ & M & GKR0613 \\
\hline $\begin{array}{l}\text { Pogostemon crassicaulis } \\
\text { (Benth.) Press }\end{array}$ & Jui-lata & $\mathrm{T}$ & $\mathrm{H}$ & $\mathrm{Fp}$ & $\mathrm{O}$ & $\begin{array}{l}\text { Fl. Sep-Dec } \\
\text { Fr. U }\end{array}$ & M & GKR2171 \\
\hline Tectona grandis L.f. & Segun & $\begin{array}{l}\mathrm{D}, \mathrm{G}, \\
\mathrm{M}, \mathrm{T}\end{array}$ & $\mathrm{T}$ & Fp, Rs & $\mathrm{C}$ & $\begin{array}{l}\text { Fl. Aug-Sep } \\
\text { Fr. U }\end{array}$ & $\mathrm{T}$ & GKR1281 \\
\hline Teucrium viscidum Blume & Unknown & M, T & $\mathrm{H}$ & $\begin{array}{l}\text { Ah, Fp, } \\
\text { Rs }\end{array}$ & $\mathrm{O}$ & $\begin{array}{l}\text { Fl. Jun-Nov } \\
\text { Fr. U }\end{array}$ & $\mathrm{W}$ & GKR1060 \\
\hline Vitex negundo L. & Nishinda & $\begin{array}{l}\mathrm{D}, \mathrm{G}, \\
\mathrm{M}, \mathrm{T}\end{array}$ & $\mathrm{S}$ & $\mathrm{Ah}$ & $\mathrm{C}$ & $\begin{array}{l}\text { Fl. Feb-Jul } \\
\text { Fr. U }\end{array}$ & M & GKR0077 \\
\hline $\begin{array}{l}\text { V. peduncularis Wall. ex } \\
\text { Schauer }\end{array}$ & $\begin{array}{l}\text { Boruna, } \\
\text { Horina }\end{array}$ & G & ST & $\mathrm{Ah}$ & $\mathrm{O}$ & $\begin{array}{l}\text { Fl. Feb-Apr } \\
\text { Fr. U }\end{array}$ & M & GKR0354 \\
\hline \multicolumn{9}{|l|}{ HYDROCHARITACEAE } \\
\hline $\begin{array}{l}\text { Hydrolea zeylanica (L.) } \\
\text { Vahl }\end{array}$ & $\begin{array}{l}\text { Kaschera, } \\
\text { Bishlanguli }\end{array}$ & $\mathrm{T}$ & $\mathrm{H}$ & $\mathrm{Ah}, \mathrm{Fp}$ & $\mathrm{O}$ & $\begin{array}{l}\text { Fl. Nov-Dec } \\
\text { Fr. U }\end{array}$ & We & GKR2203 \\
\hline Ottelia alismoides (L.) Pers. & Panikola & $\mathrm{D}$ & $\mathrm{H}$ & Fp, Rs & $\mathrm{O}$ & $\begin{array}{l}\text { Fl. Aug-Oct } \\
\text { Fr. U }\end{array}$ & $\begin{array}{l}\text { Aq } \\
\text { we }\end{array}$ & GKR1625 \\
\hline Vallisneria spiralis $\mathrm{L}$. & Patajhanji & $\mathrm{D}, \mathrm{M}$ & $\mathrm{H}$ & $\mathrm{Fp}$ & $\mathrm{O}$ & $\begin{array}{l}\text { Fl. Jun-Oct } \\
\text { Fr. U }\end{array}$ & $\begin{array}{l}\text { Aq } \\
\text { we }\end{array}$ & GKR0150 \\
\hline \multicolumn{9}{|l|}{ PLANTAGINACEAE } \\
\hline $\begin{array}{l}\text { Emilia sonchifolia (L.) DC. } \\
\text { ex DC. }\end{array}$ & Sadimodi & M & $\mathrm{H}$ & Rs & $\mathrm{O}$ & $\begin{array}{l}\text { Fl. Jul-Dec } \\
\text { Fr. U }\end{array}$ & M & GKR0209 \\
\hline $\begin{array}{l}\text { Limnophila aromatica (Lam. } \\
\text { ) Merr. }\end{array}$ & Belem & M & $\mathrm{H}$ & Rs & $\mathrm{O}$ & $\begin{array}{l}\text { Fl. Jun-Aug } \\
\text { Fr. U }\end{array}$ & Or & GKR0249 \\
\hline $\begin{array}{l}\text { Mecardonia procumbens } \\
\text { (Mill.) Small }\end{array}$ & Garurbramhi & $\begin{array}{l}\mathrm{D}, \mathrm{M}, \\
\mathrm{T}\end{array}$ & $\mathrm{H}$ & $\begin{array}{l}\text { Ah, Fp, } \\
\text { Rs }\end{array}$ & $\mathrm{O}$ & $\begin{array}{l}\text { Fl. Jun-Sep } \\
\text { Fr. U }\end{array}$ & We & GKR0075 \\
\hline $\begin{array}{l}\text { OLEACEAE } \\
\text { Jasminum officinale L. }\end{array}$ & Jui & M & $\mathrm{S}$ & $\mathrm{Ah}$ & $\mathrm{O}$ & $\begin{array}{l}\text { Fl. May-Sep } \\
\text { Fr. U }\end{array}$ & Or & GKR0385 \\
\hline
\end{tabular}

(Table contd.) 


\begin{tabular}{|c|c|c|c|c|c|c|c|c|}
\hline Scientific Name & Bangla name & District & Habit & Habitat & Occ. & Fl./Fr. Time & Use & RSE \\
\hline J. scandens (Retz.) Vahl & Paharijui & $\mathrm{G}, \mathrm{M}$ & $S$ & $\mathrm{Ah}, \mathrm{Fp}$ & $\mathrm{O}$ & $\begin{array}{l}\text { Fl. Jun-Aug } \\
\text { Fr. U }\end{array}$ & $\mathrm{W}$ & GKR0185 \\
\hline Nyctanthes arbor-tristis $\mathrm{L}$. & Shefali, Shiuli & $\begin{array}{l}\mathrm{D}, \mathrm{G}, \\
\mathrm{M}\end{array}$ & $\mathrm{T}$ & $\mathrm{Ah}, \mathrm{Fp}$ & $\mathrm{O}$ & $\begin{array}{l}\text { Fl. Sep-Oct } \\
\text { Fr. U }\end{array}$ & $\mathrm{D}$ & GKR0480 \\
\hline \multicolumn{9}{|l|}{ SCROPHULARIACEAE } \\
\hline Lindenbergia indica Vatke & Basanti & M & $\mathrm{H}$ & $\mathrm{Ah}$ & $\mathrm{O}$ & $\begin{array}{l}\text { Fl. Oct-Jan } \\
\text { Fr. U }\end{array}$ & M & GKR0179 \\
\hline $\begin{array}{l}\text { Lindernia anagallis } \\
\text { (Burm.f.) Pennell }\end{array}$ & Panighas & $\mathrm{D}, \mathrm{G}, \mathrm{T}$ & $\mathrm{H}$ & $\begin{array}{l}\text { Ah, Fp, } \\
\text { Rs }\end{array}$ & $\mathrm{O}$ & $\begin{array}{l}\text { Fl. Jul-Dec } \\
\text { Fr. U }\end{array}$ & We & GKR0426 \\
\hline L. antipoda (L.) Alston & Sada panighas & M & $\mathrm{H}$ & $\begin{array}{l}\text { Ah, Fp, } \\
\text { Rs }\end{array}$ & $\mathrm{O}$ & $\begin{array}{l}\text { Fl. Aug-Oct } \\
\text { Fr. U }\end{array}$ & We & GKR0057 \\
\hline L. crustacea (L.) F. Muell. & Chapraghash & $\mathrm{G}, \mathrm{T}$ & $\mathrm{H}$ & $\begin{array}{l}\text { Ah, Fp, } \\
\text { Rs }\end{array}$ & $\mathrm{O}$ & $\begin{array}{l}\text { Fl. Jul-Aug } \\
\text { Fr. U }\end{array}$ & We & GKR0751 \\
\hline L. elata (Benth.) Wettst. & Unknown & $\mathrm{M}, \mathrm{T}$ & $\mathrm{H}$ & $\mathrm{Fp}$ & $\mathrm{O}$ & $\begin{array}{l}\text { Fl. Jul-Oct } \\
\text { Fr. U }\end{array}$ & We & GKR0148 \\
\hline L. hyssopioides (L.) Haines & Unknown & $\mathrm{G}, \mathrm{T}$ & $\mathrm{H}$ & $\mathrm{Ah}$ & $\mathrm{O}$ & $\begin{array}{l}\text { Fl. Mar-Sep } \\
\text { Fr. U }\end{array}$ & We & GKR0235 \\
\hline L. parviflora (Roxb.) Haines & Unknown & $\mathrm{D}$ & $\mathrm{H}$ & $\mathrm{Fp}$ & $\mathrm{O}$ & $\begin{array}{l}\text { Fl. Aug-Dec } \\
\text { Fr. U }\end{array}$ & We & GKR0623 \\
\hline L. rotundifolia (L.) Alston & Unknown & $\mathrm{T}$ & $\mathrm{H}$ & $\begin{array}{l}\text { Ah, Fp, } \\
\text { Rs }\end{array}$ & $\mathrm{O}$ & $\begin{array}{l}\text { Fl. Aug-Nov } \\
\text { Fr. U }\end{array}$ & We & GKR0978 \\
\hline L. viscosa (Hornem.) Merr. & Unknown & $\mathrm{M}, \mathrm{T}$ & $\mathrm{H}$ & Rs & $\mathrm{O}$ & $\begin{array}{l}\text { Fl. Jul-Oct } \\
\text { Fr. U }\end{array}$ & We & GKR1766 \\
\hline Mazus rugosus Lour. & Unknown & $\mathrm{M}, \mathrm{T}$ & $\mathrm{H}$ & $\begin{array}{l}\text { Ah, Fp, } \\
\text { Rs }\end{array}$ & $\mathrm{O}$ & $\begin{array}{l}\text { Fl. Aug-Dec } \\
\text { Fr. U }\end{array}$ & We & GKR1048 \\
\hline Scoparia dulcis L. & $\begin{array}{l}\text { Jangli-dhone, } \\
\text { Misridana }\end{array}$ & $\begin{array}{l}\mathrm{D}, \mathrm{G}, \\
\mathrm{M}, \mathrm{T}\end{array}$ & $\mathrm{H}$ & $\begin{array}{l}\text { Ah, Fp, } \\
\text { Rs }\end{array}$ & $\mathrm{C}$ & $\begin{array}{l}\text { Fl. \& Fr. Through- } \\
\text { out the year }\end{array}$ & M & GKR0274 \\
\hline \multicolumn{9}{|l|}{ ACANTHACEAE } \\
\hline $\begin{array}{l}\text { Andrographis paniculata } \\
\text { (Burm.f.) Nees. }\end{array}$ & $\begin{array}{l}\text { Kalomegh, } \\
\text { Mohateeta }\end{array}$ & $\begin{array}{l}\mathrm{D}, \mathrm{G}, \\
\mathrm{M}\end{array}$ & $\mathrm{H}$ & $\begin{array}{l}\text { Ah, Fp, } \\
\text { Rs }\end{array}$ & $\mathrm{V}$ & $\begin{array}{l}\text { Fl. Mar-Dec } \\
\text { Fr. U }\end{array}$ & M & GKR0074 \\
\hline $\begin{array}{l}\text { Dipteracanthus patulus } \\
\text { (Jacq.) Nees }\end{array}$ & $\begin{array}{l}\text { Spreading } \\
\text { Ruellia }\end{array}$ & G & $\mathrm{H}$ & Rs & $\mathrm{O}$ & Fl. \& Fr. Oct-Feb & We & GKR2038 \\
\hline D. prostratus (Poir.) Nees & Unknown & $\mathrm{D}, \mathrm{G}$ & $\mathrm{H}$ & $\begin{array}{l}\text { Ah, Fp, } \\
\text { Rs }\end{array}$ & $\mathrm{O}$ & Fl. \& Fr. Oct-Apr & We & GKR0348 \\
\hline $\begin{array}{l}\text { Ecbolium ligustrinum (Vahl) } \\
\text { Vollesen }\end{array}$ & $\begin{array}{l}\text { Green Shrimp } \\
\text { Plant }\end{array}$ & $\mathrm{M}$ & $\mathrm{H}$ & Fp, Rs & $\mathrm{O}$ & $\begin{array}{l}\text { Fl. Jan-May } \\
\text { Fr. U }\end{array}$ & M & GKR2138 \\
\hline $\begin{array}{l}\text { Hemigraphis hirta (Vahl.) } \\
\text { T.Anderson }\end{array}$ & $\begin{array}{l}\text { Buripana, } \\
\text { Boratighas }\end{array}$ & $\begin{array}{l}\mathrm{D}, \mathrm{G}, \\
\mathrm{M}, \mathrm{T}\end{array}$ & $\mathrm{H}$ & $\begin{array}{l}\text { Ah, Fp, } \\
\text { Rs }\end{array}$ & $\mathrm{C}$ & $\begin{array}{l}\text { Fl. Jan-May } \\
\text { Fr. U }\end{array}$ & $\mathrm{W}$ & GKR0013 \\
\hline $\begin{array}{l}\text { Hygrophila polysperma } \\
\text { (Roxb.) T. Anders. }\end{array}$ & Murmuri & $\begin{array}{l}\mathrm{D}, \mathrm{G}, \\
\mathrm{M}, \mathrm{T}\end{array}$ & $\mathrm{H}$ & Fp, Rs & $\mathrm{C}$ & $\begin{array}{l}\text { Fl. Oct-Mar } \\
\text { Fr. U }\end{array}$ & Or & GKR1332 \\
\hline H. phlomoides Nees & Filamo & G & $\mathrm{H}$ & $\mathrm{Ah}$ & $\mathrm{O}$ & $\begin{array}{l}\text { Fl. Apr-Aug } \\
\text { Fr. U }\end{array}$ & We & GKR2022 \\
\hline $\begin{array}{l}\text { H. schulli (Buch.-Ham.) M. } \\
\text { R. \& S. N. }\end{array}$ & Kulekhara & G & $\mathrm{H}$ & Rs & $\mathrm{O}$ & $\begin{array}{l}\text { Fl. Sep-Apr } \\
\text { Fr. U }\end{array}$ & M & GKR0247 \\
\hline H. difformis (L.f.) Blume & Jaljanti & G & $\mathrm{H}$ & $\mathrm{Ah}$ & $\mathrm{O}$ & $\begin{array}{l}\text { Fl. Aug-Mar } \\
\text { Fr. U }\end{array}$ & We & GKR2005 \\
\hline Justicia adhatoda $\mathrm{L}$. & Bashok & $\begin{array}{l}\mathrm{D}, \mathrm{G}, \\
\mathrm{M}\end{array}$ & $S$ & $\begin{array}{l}\text { Ah, Fp, } \\
\text { Rs }\end{array}$ & $\mathrm{O}$ & Fl. \& Fr. Dec-Jun & M & GKR0737 \\
\hline J. gendarussa Burm.f. & $\begin{array}{l}\text { Jagotmadan, } \\
\text { Nilnishinda }\end{array}$ & $\begin{array}{l}\mathrm{D}, \mathrm{G}, \\
\mathrm{M}, \mathrm{T}\end{array}$ & $\mathrm{S}$ & $\begin{array}{l}\text { Ah, Fp, } \\
\text { Rs }\end{array}$ & $\mathrm{C}$ & $\begin{array}{l}\text { Fl. Jan-Apr } \\
\text { Fr. U }\end{array}$ & M & GKR 0311 \\
\hline
\end{tabular}

(Table contd.) 


\begin{tabular}{|c|c|c|c|c|c|c|c|c|}
\hline Scientific Name & Bangla name & District & Habit & Habitat & Occ. & Fl./Fr. Time & Use & RSE \\
\hline $\begin{array}{l}\text { Nelsonia canescens (Lam.) } \\
\text { Spreng. }\end{array}$ & paramul & $\mathrm{G}$ & $\mathrm{H}$ & $\mathrm{Ah}, \mathrm{Fp}$ & $\mathrm{O}$ & $\begin{array}{l}\text { Fl. Jan-Mar } \\
\text { Fr. U }\end{array}$ & $\mathrm{M}$ & GKR0236 \\
\hline $\begin{array}{l}\text { Phlogacanthus curviflorus } \\
\text { (Wall.) Nees }\end{array}$ & $\begin{array}{l}\text { Tamropuspi } \\
\text { Basok }\end{array}$ & M & $\mathrm{S}$ & $\mathrm{Fp}$ & $\mathrm{O}$ & $\begin{array}{l}\text { Fl. Oct-Feb } \\
\text { Fr. Feb-May }\end{array}$ & M & GKR0053 \\
\hline Ruellia tuberosa $\mathrm{L}$. & Patpati & $\begin{array}{l}\mathrm{D}, \mathrm{G}, \\
\mathrm{M}, \mathrm{T}\end{array}$ & $\mathrm{H}$ & $\begin{array}{l}\text { Ah, Fp, } \\
\text { Rs }\end{array}$ & $\mathrm{C}$ & $\begin{array}{l}\text { Fl. \& Fr. Through- } \\
\text { out the year }\end{array}$ & We & GKR0466 \\
\hline Rungia pectinata (L.) Nees & Pindi & $\begin{array}{l}\mathrm{D}, \mathrm{G}, \\
\mathrm{M}, \mathrm{T}\end{array}$ & $\mathrm{H}$ & $\begin{array}{l}\text { Ah, Fp, } \\
\text { Rs }\end{array}$ & $\mathrm{C}$ & $\begin{array}{l}\text { Fl. Nov-Feb } \\
\text { Fr. U }\end{array}$ & We & GKR0035 \\
\hline \multicolumn{9}{|l|}{ PEDALIACEAE } \\
\hline Sesamum indicum $\mathrm{L}$. & Til & $\mathrm{D}$ & $\mathrm{H}$ & $\begin{array}{l}\text { Ah, Fp, } \\
\text { Rs }\end{array}$ & $\mathrm{O}$ & $\begin{array}{l}\text { Fl. Jun-Jul } \\
\text { Fr. U }\end{array}$ & $\mathrm{O}$ & GKR1411 \\
\hline \multicolumn{9}{|l|}{ BIGNONIACEAE } \\
\hline Oroxylum indicum (L.) Kurz & $\begin{array}{l}\text { Sona, } \\
\text { Kanaidinga }\end{array}$ & $\begin{array}{l}\mathrm{D}, \mathrm{M}, \\
\mathrm{T}\end{array}$ & $\mathrm{T}$ & $\begin{array}{l}\text { Ah, Fp, } \\
\text { Rs }\end{array}$ & $\mathrm{O}$ & $\begin{array}{l}\text { Fl. Jul-Dec } \\
\text { Fr. U }\end{array}$ & $\begin{array}{l}\text { Fw, } \\
\mathrm{M}\end{array}$ & GKR1257 \\
\hline Tecoma gaudichaudii DC. & Hoimonti & $\mathrm{M}, \mathrm{T}$ & $\mathrm{S}$ & Rs & $\mathrm{O}$ & $\begin{array}{l}\text { Fl. Feb-Apr } \\
\text { Fr. U }\end{array}$ & Or & GKR2105 \\
\hline T. stans (L.) Juss. ex Kunth & Chandaprabha & M & $\mathrm{S}$ & Rs & $\mathrm{O}$ & $\begin{array}{l}\text { Fl. Dec-Feb } \\
\text { Fr. U }\end{array}$ & Or & GKR2093 \\
\hline \multicolumn{9}{|l|}{ RUBIACEAE } \\
\hline $\begin{array}{l}\text { Dentella repens (L.) J. R. \& } \\
\text { G. Forst. }\end{array}$ & Bhuipat & $\mathrm{D}, \mathrm{T}$ & $\mathrm{H}$ & $\mathrm{Ah}, \mathrm{Fp}$ & $\mathrm{O}$ & $\begin{array}{l}\text { Fl. Mar-Apr } \\
\text { Fr. U }\end{array}$ & We & GKR0481 \\
\hline Gardenia jasminoides J.Ellis & Gandharaj & $\begin{array}{l}\mathrm{D}, \mathrm{G}, \\
\mathrm{M}, \mathrm{T}\end{array}$ & $\mathrm{S}$ & $\mathrm{Ah}$ & $\mathrm{C}$ & $\begin{array}{l}\text { Fl. Apr-Aug } \\
\text { Fr. U }\end{array}$ & Or & GKR1011 \\
\hline $\begin{array}{l}\text { Hedyotis axillaris Gardner } \\
\text { ex Thwaites }\end{array}$ & Unknown & $\mathrm{D}, \mathrm{T}$ & $\mathrm{H}$ & $\mathrm{Ah}, \mathrm{Fp}$ & $\mathrm{O}$ & $\begin{array}{l}\text { Fl. Jul-Oct } \\
\text { Fr. U }\end{array}$ & We & GKR0170 \\
\hline H. racemosa Lam. & Unknown & M & $\mathrm{H}$ & $\mathrm{Ah}, \mathrm{Fp}$ & $\mathrm{O}$ & $\begin{array}{l}\text { Fl. Jan-Sep } \\
\text { Fr. U }\end{array}$ & We & GKR0071 \\
\hline H. hermanniana R.M.Dutta & Unknown & M & $\mathrm{H}$ & Ah, Rs & $\mathrm{O}$ & $\begin{array}{l}\text { Fl. Jun-Dec } \\
\text { Fr. U }\end{array}$ & We & GKR0020 \\
\hline Ixora coccinea $\mathrm{L}$. & Rangon & $\begin{array}{l}\mathrm{G}, \mathrm{M}, \\
\mathrm{T}\end{array}$ & $\mathrm{S}$ & $\mathrm{Fp}, \mathrm{Rs}$ & $\mathrm{O}$ & $\begin{array}{l}\text { Fl. \& Fr. Through- } \\
\text { out the year }\end{array}$ & $\begin{array}{l}\mathrm{M}, \\
\text { Or }\end{array}$ & GKR0464 \\
\hline $\begin{array}{l}\text { Meyna pubescens (Kurz) } \\
\text { Robyns }\end{array}$ & Mainphal & $\mathrm{M}, \mathrm{T}$ & $\mathrm{S}$ & Fp, Rs & $\mathrm{O}$ & $\begin{array}{l}\text { Fl. Mar-Apr } \\
\text { Fr. U }\end{array}$ & $\mathrm{W}$ & GKR2140 \\
\hline $\begin{array}{l}\text { Neolamarckia cadamba } \\
\text { (Roxb.) Bosser }\end{array}$ & Kadam & $\begin{array}{l}\mathrm{D}, \mathrm{G}, \\
\mathrm{M}, \mathrm{T}\end{array}$ & $\mathrm{T}$ & Fp, Rs & $\mathrm{C}$ & $\begin{array}{l}\text { Fl. Apr-Aug } \\
\text { Fr. U }\end{array}$ & $\begin{array}{l}\mathrm{O}, \\
\mathrm{Pi}\end{array}$ & GKR1209 \\
\hline Oldenlandia corymbosa $\mathrm{L}$. & Khet papra & $\begin{array}{l}\mathrm{D}, \mathrm{G}, \\
\mathrm{M}, \mathrm{T}\end{array}$ & $\mathrm{H}$ & $\begin{array}{l}\text { Ah, Fp, } \\
\text { Rs }\end{array}$ & $\mathrm{C}$ & $\begin{array}{l}\text { Fl. Jul-Aug } \\
\text { Fr. U }\end{array}$ & We & GKR0471 \\
\hline O. diffusa (Willd.) Roxb. & Pitpapra & $\mathrm{D}, \mathrm{G}, \mathrm{T}$ & $\mathrm{H}$ & $\begin{array}{l}\text { Ah, Fp, } \\
\text { Rs }\end{array}$ & $\mathrm{O}$ & $\begin{array}{l}\text { Fl. Aug-Nov } \\
\text { Fr. U }\end{array}$ & We & GKR0862 \\
\hline O. biflora L. & Damanpapra & $\mathrm{M}, \mathrm{T}$ & $\mathrm{H}$ & $\begin{array}{l}\text { Ah, Fp, } \\
\text { Rs }\end{array}$ & $\mathrm{O}$ & $\begin{array}{l}\text { Fl. \& Fr. Through- } \\
\text { out the year }\end{array}$ & We & GKR0500 \\
\hline $\begin{array}{l}\text { Ophiorrhiza harrisiana var. } \\
\text { rugosa (Wall.) Hook. f. }\end{array}$ & $\begin{array}{l}\text { Jari Ful, } \\
\text { Kalashona }\end{array}$ & $\mathrm{D}, \mathrm{T}$ & $\mathrm{H}$ & Rs & $\mathrm{O}$ & $\begin{array}{l}\text { Fl. Jun-Aug } \\
\text { Fr. U }\end{array}$ & M & GKR0665 \\
\hline Paederia foetida $\mathrm{L}$. & $\begin{array}{l}\text { Gandhyabhadu } \\
\text { li }\end{array}$ & M & $\mathrm{H}$ & Rs & $\mathrm{O}$ & $\begin{array}{l}\text { Fl. Jun-Aug } \\
\text { Fr. U }\end{array}$ & M & GKR0218 \\
\hline Pavetta indica $\mathrm{L}$. & $\begin{array}{l}\text { Kukurchura/Ju } \\
\text { i }\end{array}$ & $\mathrm{M}, \mathrm{T}$ & $\mathrm{S}$ & Ah, Rs & $\mathrm{O}$ & $\begin{array}{l}\text { Fl. Apr-Jul } \\
\text { Fr. U }\end{array}$ & Or & GKR2915 \\
\hline $\begin{array}{l}\text { Psilanthus bengalensis } \\
\text { (Roxb.) Leroy }\end{array}$ & Ban Coffe & $\mathrm{D}, \mathrm{G}, \mathrm{T}$ & $\mathrm{S}$ & Rs & $\mathrm{O}$ & $\begin{array}{l}\text { Fl. Feb-May } \\
\text { Fr. U }\end{array}$ & M & GKR0333 \\
\hline
\end{tabular}

(Table contd.) 


\begin{tabular}{|c|c|c|c|c|c|c|c|c|}
\hline Scientific Name & Bangla name & District & Habit & Habitat & Occ. & Fl./Fr. Time & Use & RSE \\
\hline Richardia scabra L. & Taraphul & $\mathrm{D}, \mathrm{M}$ & $\mathrm{H}$ & $\begin{array}{l}\text { Ah, Fp, } \\
\text { Rs }\end{array}$ & $\mathrm{O}$ & $\begin{array}{l}\text { Fl. Nov-Feb } \\
\text { Fr. U }\end{array}$ & $\mathrm{We}$ & GKR0056 \\
\hline Spermacoce articularis L. f. & Bhagajongla & G & $\mathrm{H}$ & $\mathrm{Fp}, \mathrm{Rs}$ & $\mathrm{O}$ & $\begin{array}{l}\text { Fl. Oct-Dec } \\
\text { Fr. U }\end{array}$ & M & GKR0357 \\
\hline $\begin{array}{l}\text { S. exilis (L.O.Williams) } \\
\text { C.D.Adams ex W.C.Burger } \\
\text { \& C.M.Taylor }\end{array}$ & Unknown & G & $\mathrm{H}$ & $\mathrm{Ah}, \mathrm{Fp}$ & $\mathrm{O}$ & $\begin{array}{l}\text { Fl. Sep-Oct } \\
\text { Fr. U }\end{array}$ & $\mathrm{W}$ & GKR0836 \\
\hline S. latifolia Aubl. & Ghuijil & $\mathrm{D}, \mathrm{G}$ & $\mathrm{H}$ & Fp, Rs & $\mathrm{O}$ & $\begin{array}{l}\text { Fl. Aug-Oct } \\
\text { Fr. U }\end{array}$ & $\mathrm{W}$ & GKR0840 \\
\hline \multicolumn{9}{|l|}{ ASTERACEAE } \\
\hline $\begin{array}{l}\text { Acmella radicans (Jacq.) } \\
\text { R.K.Jansen }\end{array}$ & Surjakonnya & $\mathrm{G}, \mathrm{M}$ & $\mathrm{H}$ & $\begin{array}{l}\text { Ah, Fp, } \\
\text { Rs }\end{array}$ & $\mathrm{O}$ & $\begin{array}{l}\text { Fl. Sep-Jan } \\
\text { Fr. U }\end{array}$ & M & GKR1093 \\
\hline Ageratum conyzoides $\mathrm{L}$. & Phulkuri & $\begin{array}{l}\mathrm{D}, \mathrm{G}, \\
\mathrm{M}, \mathrm{T}\end{array}$ & $\mathrm{H}$ & $\begin{array}{l}\text { Ah, Fp, } \\
\text { Rs }\end{array}$ & $\mathrm{C}$ & $\begin{array}{l}\text { Fl. \& Fr. Through- } \\
\text { out the year }\end{array}$ & M & GKR0165 \\
\hline $\begin{array}{l}\text { Blumea lacera (Burm.f.) } \\
\text { DC. }\end{array}$ & $\begin{array}{l}\text { Shealmutra, } \\
\text { Shealmoti }\end{array}$ & $\begin{array}{l}\mathrm{D}, \mathrm{G}, \\
\mathrm{M}\end{array}$ & $\mathrm{H}$ & $\begin{array}{l}\text { Ah, Fp, } \\
\text { Rs }\end{array}$ & $\mathrm{O}$ & $\begin{array}{l}\text { Fl.\& Fr. } \\
\text { Throughout the } \\
\text { year }\end{array}$ & M & GKR0063 \\
\hline Caesulia axillaris Roxb. & Fuiltaghas & $\begin{array}{l}\mathrm{D}, \mathrm{M}, \\
\mathrm{T}\end{array}$ & $\mathrm{H}$ & $\mathrm{Fp}, \mathrm{Rs}$ & $\mathrm{O}$ & $\begin{array}{l}\text { Fl. Sep-Nov } \\
\text { Fr. U }\end{array}$ & We & GKR0196 \\
\hline $\begin{array}{l}\text { Chromolaena odorata (L.) } \\
\text { R.M.King \& H.Rob. }\end{array}$ & Assamlata & $\mathrm{D}$ & $\mathrm{S}$ & $\mathrm{Fp}$ & $\mathrm{O}$ & $\begin{array}{l}\text { Fl. Nov-May } \\
\text { Fr. U }\end{array}$ & M & GKR0673 \\
\hline Eclipta alba (L.) Hassk. & $\begin{array}{l}\text { Kalokeshi, } \\
\text { Kesaraj }\end{array}$ & $\begin{array}{l}\mathrm{D}, \mathrm{G}, \\
\mathrm{M}, \mathrm{T}\end{array}$ & $\mathrm{H}$ & $\begin{array}{l}\text { Ah, Fp, } \\
\text { Rs }\end{array}$ & $\mathrm{C}$ & $\begin{array}{l}\text { Fl. Aug-Sep, } \\
\text { Fr. Oct-Dec }\end{array}$ & M & GKR0459 \\
\hline Elephantopus scaber L. & Hastipadi & $\mathrm{D}, \mathrm{G}$ & $\mathrm{H}$ & $\mathrm{Fp}, \mathrm{Rs}$ & $\mathrm{O}$ & $\begin{array}{l}\text { Fl. Oct-Jan } \\
\text { Fr. U }\end{array}$ & M & GKR0244 \\
\hline Gnaphalium luteo-album L. & Bara Kamra & G & $\mathrm{H}$ & Rs & $\mathrm{O}$ & $\begin{array}{l}\text { Fl. Jun-Aug } \\
\text { Fr. U }\end{array}$ & We & GKR0427 \\
\hline G. pensylvanicum Willd. & Silvalomi & M & $\mathrm{H}$ & Rs & $\mathrm{O}$ & $\begin{array}{l}\text { Fl. Dec-Jul } \\
\text { Fr. U }\end{array}$ & We & GKR0101 \\
\hline $\begin{array}{l}\text { Grangea maderaspatana } \\
\text { (L.) Poir. }\end{array}$ & Nimuti & $\begin{array}{l}\mathrm{D}, \mathrm{G}, \\
\mathrm{M}\end{array}$ & $\mathrm{H}$ & $\begin{array}{l}\text { Ah, Fp, } \\
\text { Rs }\end{array}$ & $\mathrm{O}$ & $\begin{array}{l}\text { Fl. Jan-Aug } \\
\text { Fr. U }\end{array}$ & We & GKR0157 \\
\hline $\begin{array}{l}\text { Gynura procumbens (Lour.) } \\
\text { Merr. }\end{array}$ & Diabetes plant & M & $\mathrm{H}$ & $\mathrm{Ah}$ & $\mathrm{O}$ & $\begin{array}{l}\text { Fl. Mar-Apr } \\
\text { Fr. U }\end{array}$ & M & GKR1957 \\
\hline $\begin{array}{l}\text { Mikania cordata (Burm. f.) } \\
\text { B.L.Rob. }\end{array}$ & Assam lata & $\begin{array}{l}\mathrm{D}, \mathrm{G}, \\
\mathrm{M}, \mathrm{T}\end{array}$ & $\mathrm{H}$ & $\begin{array}{l}\text { Ah, Fp, } \\
\text { Rs }\end{array}$ & $\mathrm{C}$ & $\begin{array}{l}\text { Fl. Jun-Aug } \\
\text { Fr. U }\end{array}$ & M & GKR0434 \\
\hline M. scandens (L.) Willd. & climbing hemp & $\mathrm{G}, \mathrm{M}$ & $\mathrm{H}$ & $\mathrm{Ah}$ & $\mathrm{O}$ & $\begin{array}{l}\text { Fl. Jun-Aug } \\
\text { Fr. U }\end{array}$ & $\begin{array}{l}\mathrm{Fd}, \\
\text { Or }\end{array}$ & GKR0399 \\
\hline $\begin{array}{l}\text { Parthenium hysterophorus } \\
\text { L. }\end{array}$ & Bish gach & M & $\mathrm{H}$ & Rs & $\mathrm{O}$ & $\begin{array}{l}\text { Fl. Jun-Sep } \\
\text { Fr. U }\end{array}$ & $\mathrm{P}$ & GKR1228 \\
\hline $\begin{array}{l}\text { Pseudelephantopus spicatus } \\
\text { (B.Juss. ex Aubl.) Rohr ex } \\
\text { C.F.Baker }\end{array}$ & Dog's-tongue & $\begin{array}{l}\mathrm{G}, \mathrm{M}, \\
\mathrm{T}\end{array}$ & $\mathrm{H}$ & $\begin{array}{l}\text { Ah, Fp, } \\
\text { Rs }\end{array}$ & $\mathrm{O}$ & $\begin{array}{l}\text { Fl. Oct-Dec } \\
\text { Fr. U }\end{array}$ & We & GKR2031 \\
\hline Sonchus arvensis $\mathrm{L}$. & Dhudia & $\mathrm{M}$ & $\mathrm{H}$ & $\mathrm{Fp}$ & $\mathrm{O}$ & $\begin{array}{l}\text { Fl. Jul-Oct } \\
\text { Fr. U }\end{array}$ & $\mathrm{W}$ & GKR0103 \\
\hline Spilanthes calva DC. & Marhatitiga & $\begin{array}{l}\mathrm{D}, \mathrm{M}, \\
\mathrm{T}\end{array}$ & $\mathrm{H}$ & $\mathrm{Fp}, \mathrm{Rs}$ & $\mathrm{O}$ & $\begin{array}{l}\text { Fl. Feb-Apr } \\
\text { Fr. U }\end{array}$ & M & GKR1128 \\
\hline S. acmella (L.) L. & Surjakonnya & $\begin{array}{l}\mathrm{D}, \mathrm{G}, \\
\mathrm{M}, \mathrm{T}\end{array}$ & $\mathrm{H}$ & $\begin{array}{l}\text { Ah, Fp, } \\
\text { Rs }\end{array}$ & $\mathrm{C}$ & $\begin{array}{l}\text { Fl. Jun-Sep } \\
\text { Fr. U }\end{array}$ & M & GKR0675 \\
\hline $\begin{array}{l}\text { Synedrella nodiflora (L.) } \\
\text { Gaertn. }\end{array}$ & Nakphul & $\begin{array}{l}\mathrm{D}, \mathrm{G}, \\
\mathrm{M}, \mathrm{T}\end{array}$ & $\mathrm{H}$ & $\begin{array}{l}\text { Ah, Fp, } \\
\text { Rs }\end{array}$ & $\mathrm{C}$ & $\begin{array}{l}\text { Fl.\& Fr. Through- } \\
\text { out the year }\end{array}$ & We & GKR0474 \\
\hline
\end{tabular}

(Table contd.) 


\begin{tabular}{|c|c|c|c|c|c|c|c|c|}
\hline Scientific Name & Bangla name & District & Habit & Habitat & Occ. & Fl./Fr. Time & Use & RSE \\
\hline Tagetes erecta $\mathrm{L}$. & Ganda & $\mathrm{D}, \mathrm{M}$ & $\mathrm{H}$ & $\mathrm{Fp}$ & $\mathrm{O}$ & $\begin{array}{l}\text { Fl. Nov-Mar } \\
\text { Fr. U }\end{array}$ & Or & GKR1890 \\
\hline Tridax procumbens (L.) L. & Tridhara & $\mathrm{D}, \mathrm{G}, \mathrm{T}$ & $\mathrm{H}$ & $\begin{array}{l}\text { Ah, Fp, } \\
\text { Rs }\end{array}$ & $\mathrm{O}$ & $\begin{array}{l}\text { Fl.\& Fr. Through- } \\
\text { out the year }\end{array}$ & $\mathrm{We}$ & GKR0484 \\
\hline Vernonia cinerea $($ L.) Less. & Kukurshunga & $\begin{array}{l}\mathrm{D}, \mathrm{G}, \\
\mathrm{M}, \mathrm{T}\end{array}$ & $\mathrm{H}$ & $\begin{array}{l}\text { Ah, Fp, } \\
\text { Rs }\end{array}$ & $\mathrm{C}$ & $\begin{array}{l}\text { Fl.\& Fr. Through- } \\
\text { out the year }\end{array}$ & We & GKR0064 \\
\hline Wedelia biflora (L.) DC. & $\begin{array}{l}\text { Beach } \\
\text { Sunflower }\end{array}$ & $\mathrm{D}$ & $\mathrm{H}$ & $\mathrm{Fp}$ & $\mathrm{O}$ & $\begin{array}{l}\text { Fl.\& Fr. Through- } \\
\text { out the year }\end{array}$ & Or & GKR0666 \\
\hline W. chinensis (Osbeck) Merr. & Mahavringaraj & $\mathrm{D}$ & $\mathrm{H}$ & Fp, Rs & $\mathrm{O}$ & $\begin{array}{l}\text { Fl.\& Fr. Through- } \\
\text { out the year }\end{array}$ & Or & GKR0654 \\
\hline $\begin{array}{l}\text { Xanthium indicum Koen. ex } \\
\text { Roxb. }\end{array}$ & $\begin{array}{l}\text { Ghagra, } \\
\text { Banokra }\end{array}$ & $\begin{array}{l}\mathrm{D}, \mathrm{G}, \\
\mathrm{M}\end{array}$ & $\mathrm{H}$ & Fp, Rs & $\mathrm{O}$ & $\begin{array}{l}\text { Fl. Mar-Dec } \\
\text { Fr. U }\end{array}$ & M & GKR0517 \\
\hline Youngia japonica (L.) DC. & Unknown & $\mathrm{M}, \mathrm{T}$ & $\mathrm{H}$ & Fp, Rs & $\mathrm{O}$ & $\begin{array}{l}\text { Fl. Feb-Dec } \\
\text { Fr. U }\end{array}$ & Or & GKR0195 \\
\hline
\end{tabular}

Legend: District: $\mathrm{D}=$ Dhaka, $\mathrm{G}=$ Gazipur, $\mathrm{M}=$ Manikganj, $\mathrm{T}=$ Tangail; Habit: $\mathrm{H}=$ Herb, $\mathrm{S}=$ Shrub, $\mathrm{T}$ = Tree; Habitat: $\mathrm{Rs}=\mathrm{Road}$ side, $\mathrm{Ah}=$ Adjoining open area except house yard, $\mathrm{Bb}=$ Bamboo bush, $\mathrm{Hy}=$ House yard, Ep $=$ Edge of the pond, $\mathrm{Fp}=$ Fallow place; Occ. $(=$ Occurance $): \mathrm{C}=$ Common, $\mathrm{CE}=$ Critically Endangered, $\mathrm{E}=$ Endangered, $\mathrm{O}=$ Occasional, $\mathrm{R}=$ Rare, $\mathrm{V}=$ Vulnerable; Fl./Fr. Time: Fl. = Flowering time, Fr. = Fruiting time, $\mathrm{U}=$ Unknown; Use: $\mathrm{Bb}=$ Boatbuilding, $\mathrm{C}=$ Cerals, $\mathrm{Ch}=$ Chutney, $\mathrm{Cw}=$ Cabinet work, $\mathrm{D}=$ Dye, $\mathrm{Fb}=$ Fiber, Fr. $=$ Fruit, Fw $=$ Fuel wood, $\mathrm{R}=$ Rubber, $\mathrm{Re}=\mathrm{Resin}, \mathrm{Rp}=$ Rice pounders, $\mathrm{T}=$ Timber, $\mathrm{W}=$ Wild, $\mathrm{We}=$ Weed, $\mathrm{M}=$ Medicinal, $\mathrm{Mi}=$ Musical instrument, Aq we $=$ Aquatic weed, $\mathrm{P}=$ Poisonus, $\mathrm{Pi}=$ Perfume industry, $\mathrm{S}=$ Spice, $\mathrm{O}=$ Oil, $\mathrm{Or}=$ Ornamental, $\mathrm{Fd}=\mathrm{Fodder}, \mathrm{H}=$ Hedge, V = Vegetable, Wo-Wood; RSE = Representative Specimens Examined (All housed in JUH).

were dominated by the herbs (238 species; $52.31 \%$ ) followed by trees (129 species; $28.35 \%$ ) and shrubs ( 88 species; $19.34 \%$ ). Among these species, 47 were climbers, six vines and four parasites. These data indicate that the homestead areas of central region of Bangladesh still harbour a large number of Dicot species and they can serve as the hotspots of the flora and biodiversity of Bangladesh.

The taxonomic enumeration of the species of dicotyledons in the homestead areas of the four districts reported by this study seems lower than the record (605 species) of Tabassum (2015). This is due to the reason that Tabassum (2015) studied this plant group of whole Gazipur district consists of homestead and different non-homestead areas, the total land area of this district is much higher than that of the 1120 homesteads of four districts under this study, and in these homesteads, a good number of the species were overlapping and common. Most of other relevant studies conducted in plain land areas of this country mention the taxonomic account of Angiosperm species instead of dicot species. The enumeration of Angiosperm species by few of these studies (Kabir and Webb, 2009) are relatively higher, whereas, that of few other studies (Begum et al., 2013; Islam et al., 2013; Muhammed et al., 2011, 2013; Rahman et al., 2009) are relatively lower than that of the dicot species by this study.

In the homesteads of the study area, Fabaceae with 41 species of 28 genera was recognized as the largest family that was followed by Euphorbiaceae with 34 species under 19 genera and Asteraceae with 26 species belonging to 22 genera. Solanum L. and Lindernia All. with eight species each was found as the largest genus in the study area, which was followed by Ficus L and Euphorbia L. with seven species each, Persicaria Mill. with six species and Albizia Durazz., Diospyros L. and Piper L. with five species each.

The composition and distribution of dicot species in the homestead areas of the four districts were remarkably variable. In the homesteads of Dhaka, Gazipur, Manikganj and Tangail districts, the occurance of total 283, 243, 285 and 223 species, respectively, was confirmed. Among these species, a total of $52,35,53$ and 20 species were found to occur exclusively in the homesteads of 
Dhaka, Gazipur, Manikganj and Tangail, respectively. Whereas, total 231, 208, 231 and 203 species, respectively of Dhaka, Gazipur, Manikganj and Tangail districts, were recorded as overlapping in the remaining three districts. Only 110 species were commonly found in the homesteads of four districts.

The similarity between and among the four districts in species composition of their homesteads measured by Jaccard coefficient shows that (Fig. 2), it is more than $60 \%$ in between any two of these districts, whereas, it is only $41 \%$ if all of the four districts are considered. The highest similarity in species composition (72\%) was found in between Gazipur and Tangail districts, and the lowest (63\%) in Dhaka and Manikganj districts.

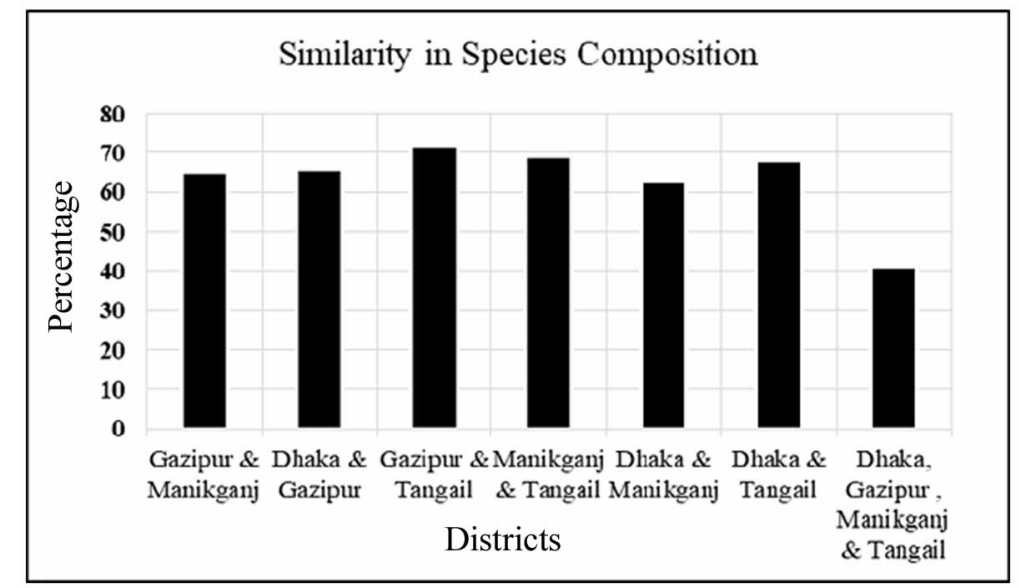

Fig. 2. Similarity in species composition in homesteads of the four districts based on Jaccard coefficient (Jaccard, 1912).

The study has recognized a total of 332 species from the homesteads of the study area of central Bangladesh as economically useful. The major categories of these economically useful species were medicinal (184 species), timber (34 species), fruit (38 species), ornamental (36 species), vegetable (30 species), fuel wood (26 species), oil (14 species), spice (five species) and fiber (eight species). Among the economically useful species of the study area, total 53 species were useful in two and seven species in three economic categories.

Among these, at least 96 tree species (e.g. Albizia lebbeck, Alstonia scholaris, Aphanamixis polystachya, Barringtonia acutangula, Bombax ceiba, Lagerstroemia speciosa, Lannea coromandelica, Miliusa velutina, Polyalthia longifolia, Syzyzium cumini, Tamarindus indica, Tectona grandis, and Trema orientalis), used as wood or timber, are now native to Bangladesh. Moreover, a good number of medicinal (e.g. Abroma augusta, Azadirachta indica, Cinnamomum tamala, C. zeylanicum, Ficus racemosa, F. religiosa, Holarrhena antidysenterica, Moringa oleifera, Ocimum sanctum, Ricinus communis, Terminalia arjuna, T. belerica, T. chebula, Tiliacora acuminata, Tinospora crispa, Vitex negundo, and Zanthoxylum rhetsa) and fruit yielding (e.g. Aegle marmelos, Annona reticulata, A. squamosa, Artocarpus heterophyllus, Averrhoa carambola, Baccaurea motleyana, Carica papaya, Citrus grandis, C. limon, Dillenia indica, Diospyros peregrina, Flacourtia jangomas, Mangifera indica, Manilkara zapota, Elaeocarpus floribundus, Phyllanthus acidus, P. reticulatus, Psidium guajava, Punica granatum, Syzygium cumini, and Tamarindus indica) species, harboured in the homesteads of the study area, are also 
native to Bangladesh. The occurrence of a good number of timber and fruit species in the homestead areas of the study area is supported by Bashar (1999). But in contrast, the population of about $28 \%$ of the native tree species (e.g. Holarrhena antidysenterica, Terminalia belerica, $T$. arjuna, Diospyros montana, Pithecellobium dulce, Macaranga indica, Mallotus philippensis, Elaeocarpus tectorius, Piper peploides, Tabernaemontana dichotoma, Miliusa velutina, Diospyros peregrina) were found to be declining in the homestead areas of the four districts.

This study has provided field data on the current status of seven threatened species, included in the Red Data Book of Vascular Plants of Bangladesh (Khan et al., 2001; Ara et al., 2013), in the study area (Table 2). The existing population size, number of localities, and estimated Extent of Occurrence (EOO) and Area of Occupancy (AOO) of these species were very narrow (Table 2). Moreover, all of these species were observed with poor regeneration and their localities were under the threats of continuous habitat destruction. Based on these facts, Abroma augusta (L.) L.f., Aniseia martinicensis (Jacq.) Choisy, Pterocarpus santalinus L.f. and Tournefortia roxburghii C.B. Clarke have been estimated as Critically Endangered (CE), Mucuna bracteata DC. ex Kurz and Rauvolfia serpentina (L.) Benth. ex Kurz as Endangered (E), and Andrographis paniculata (Burm.f.) Nees. as Vulnerable (V) in the homeatead areas of Dhaka, Gazipur, Manikganj and Tangail districts.

Table 2. Estimated threatened status of seven dicot species included in Red Data Book Bangladesh in the homestead areas of Dhaka, Gazipur, Manikganj and Tangail districts.

\begin{tabular}{|c|c|c|c|c|c|c|}
\hline Species name & $\begin{array}{l}\text { Total no. of } \\
\text { individuals }\end{array}$ & $\begin{array}{l}\text { No. of } \\
\text { locality }\end{array}$ & District & $\begin{array}{l}\mathrm{EOO} \\
\left(\mathrm{km}^{2}\right)\end{array}$ & $\begin{array}{l}\mathrm{AOO} \\
\left(\mathrm{km}^{2}\right)\end{array}$ & $\begin{array}{l}\text { Estimated threatened } \\
\text { category }\end{array}$ \\
\hline Abroma augusta & 04 & 01 & Tangail & 28 & 08 & Critically endangered \\
\hline $\begin{array}{l}\text { Andrographis } \\
\text { paniculata }\end{array}$ & 450 & 07 & $\begin{array}{l}\text { Dhaka, Gazipur } \\
\text { and Manikganj }\end{array}$ & 1040 & 35 & Vulnerable \\
\hline Aniseia martinicensis & 05 & 01 & Manikganj & 35 & 09 & Critically endangered \\
\hline Mucuna bracteata & 45 & 02 & Dhaka & 38 & 20 & Endangered \\
\hline $\begin{array}{l}\text { Pterocarpus } \\
\text { santalinus }\end{array}$ & 01 & 01 & Manikganj & 24 & 06 & Critically endangered \\
\hline Rauvolfia serpentina & 144 & 02 & $\begin{array}{l}\text { Dhaka and } \\
\text { Manikganj }\end{array}$ & 270 & 10 & Endangered \\
\hline $\begin{array}{l}\text { Tournefortia } \\
\text { roxburghii }\end{array}$ & 01 & 01 & Manikganj & 24 & 06 & Critically endangered \\
\hline
\end{tabular}

This study provides basic taxonomic information on all species of dicotyledones including seven threatened species currently occurring in the homesteads area of central region of Bangladesh, which might serve as an important baseline to track the trend of changes in the floristic composition and biodiversity conservation in the homestead areas in course of time and different biogeographical processes. The data provided by this study might be useful in planning, management, conservation and sustainable development of homestead plant genetic resources in Bangladesh.

The increased human population and associated development activities in the last few decades has resulted directly in depletion of natural vegetation, which in turn increase the pressure on the homestead forest especially in the developing countries to meet various needs of the human beings (Alam et. al., 2005). This observation is found to be true in the homestead areas of the four 
districts studied. Additionally, habitat fragmentation and depletion, intrusion of exotic (e.g. Parthenium hysterophorus, Mikania cordata, Chromolaena odorata, Acacia auriculiformis and Eucalyptus camaldulensis) and parasitic species (e.g. Cuscuta reflexa, Dendrophthoe falcata, and Helixanthera cylindrica), vegetation clearing, unnecessary firing, unplanned agricultural extension, over exploitation of natural resources, lack of awareness in the local people including the homestead owners, and lack of proper management and protection systems homestead forests etc. are also the functional threats to the homestead flora, especially the threatened and declining native plant species, of the study area.

Therefore, appropriate planning, management strategies and measures, and awareness building programs should be effectively launched for the conservation of plant genetic resources in the homestead areas of this region. Performing studies on the natural regeneration, biogeography, and pollination, breeding and dispersal mechanisms of the threatened species threatened and declining native species of the region are very crucial for their sustainable conservation. Besides, effective attempts including the provision of incentives for involving the local people in conservation programs in the region is highly recommended.

\section{Acknowledgements}

The authors are grateful to the authorities of the Bangabandhu Fellowship on Science and ICT Project for funding this study as a part of first Author's PhD program, Ministry of Science and Technology, Bangladesh Forest Department and Bangladesh National Herbarium (DACB) for their cooperation during conducting this study. The authors are thankful to the Chief Editor and the Reviewers of the Journal for their critical review of the manuscript.

\section{References}

Abedin, M.Z. and Quddus, M.A. 1990. Household fuel situation, Homegarden and agroforestry practice at six ageo-ecologically different locations of Bangladesh. In: Abedin, M. Z.; C. K. Lai and M. A. Ali (Eds), Homestead plantation and Agroforestry in Bangladesh, BARI, Gazipur, Bangladesh, pp 19-53.

Ahmed, Z.U., Hassan, M.A., Begum, Z.N.T., Khondker, M., Kabir, S.M.H., Ahmad, M., Ahmed, A.T.A., Rahman, A.K.A. and Haque, E.U. (Eds). 2008-2009. Encyclopedia of Flora and Fauna of Bangladesh, Vols. 6-10, 12. Asiatic Society of Bangladesh, Dhaka.

Alam M., 2011. Tropical homegardens in Bangladesh: Characteristics and sustainability. In: Lichtfouse E. (Ed), Alternative Farming Systems, Biotechnology. Drought Stress and Ecological Fertilisation Springer, pp. 245-262.

Alam, M.S., M.A. Hassan and Uddin, M.Z. 2006. A Preliminary Checklist of the Angiospermic flora of Ghagotia Union under Kapasia Upazila in Gazipur District, Bangladesh. Bangladesh J. Plant Taxon. 13(2): 155-170.

Alam, M.S., Masum, K.M., Campus, B.F.R.I. and Sholashahor, B. 2005. Status of homestead biodiversity in the offshore island of Bangladesh. Research Journal of Agriculture and Biological Sciences 1(3): 246-253.

Ara, H., Khan, B. and Uddin, S.N. (Eds). 2013. Red Data Book of Vascular Plants of Bangladesh. Bangladesh National Herbarium, Dhaka, pp. 1-280.

Bashar, M.A., 1999. Homegarden Agroforestry: Impact on Biodiversity conservation and household food security (A case study of Gajipur district, Bangladesh). M.Sc. thesis, Agricultural University of Norway, pp. 21-34.

Begum, M., Haque, M.A., Karim, M.R., Akter, M. and Wadud, M.A. 1913. Study on homestead Agroforestry and plant diversity in Gopalpur upazila of Tangail district. J. Agrofor. Environ. 7(1): 135-138.

Cronquist, A. 1981. An Integrated System of Classification of Flowering Plants. Columbia University Press, New York, pp. 1-1262. 
FAO, 2010. Global Forest Resource Assessment. Main Report, FAO Forestry Paper 163, Food and Agriculture Organization (FAO) of the United Nations, Rome, Italy, pp. 1-340.

Heywood, V.H. 1993. Flowering plants of the world, $2^{\text {nd }}$ Edition. B.T. Batsford Ltd., pp. 1-424.

Hooker, J.D. 1872-1897. The Flora of British India, Vols. 1-7. L. Reeve \& Co., Ashford, Kent.

Huq, A.M. 1986. Preliminary studies on the anthropogenic flora of Kutubdia Island in Bangladesh. J Asiatic Soc. Bangladesh (Sci.), 12: 59-70.

Huq, A.M. 1988. A Preliminary taxonomic report on the Angiospermic flora of Hatia Island (Noakhali district) (Dicotyledons). Bull. Bangladesh Nat. Herb. 1: 1-10.

International Plant Names Index, 2015. Published on the Internet http://www.ipni.org, The Royal Botanic Gardens, Kew, Harvard University Herbaria \& Libraries and Australian National Botanic Gardens. Accessed on 20 December 2015.

Islam, S.A., Miah, A.Q., Habib, M.A. and Moula, M.G., 2015. Enrichment of Homestead Vegetation through Agroforestry Practices in the Remote Coastal Areas of Bangladesh. Bangladesh Res. Pub. J. 11(4): 276-283.

Islam, S.A., Miah, M.A.Q. and Habib, M.A., 2013. Diversity of fruit and timber tree species in the coastal homesteads of southern Bangladesh. Journal of the Asiatic Society of Bangladesh, Science, 39(1): 83-94.

IUCN Standards and Petitions Committee. 2019. Guidelines for Using the IUCN Red List Categories and Criteria. Version 14. Prepared by the Standards and Petitions Committee, https://www. iucnredlist.org/ resources/redlistguidelines, pp. 1-113.

Jaccard, P. 1912. The distribution of the flora of the alpine zone, New Phytologist 11: 37-50.

Jain, S.K. and Rao, R.R. 1977. A Handbook of Field and Herbarium Methods. Today and Tomorrows Printers and Publishers, pp. 1-157.

Kabir, M.E. and Webb, E.L., 2009. Household and homegarden characteristics in southwestern Bangladesh. Agroforestry Systems 75(2): 129.

Khan, M.S. and Afza, S.K. 1968. A taxonomic report on the angiospermic flora of Teknaf and St. Martin's Island. Dhaka Univ. Stud., B. 16: 25-30.

Khan, M.S. and Banu, F. 1969. A taxonomic report on the angiospermic flora of Chittagong Hill Tracts-1 (Monocotyledons). Journal of Asiatic Society Pakistan 14(2): 217-224

Khan, M.S., Huq, A. M., and Rahman, M. M. 1985. Studies on the angiospermic flora of Nijhum Dwip (Char Osman) in the Bay of Bengal. Dhaka Univ. Stud., B. 33: 145-151.

Khan, M.S., Huq, M. A., Rahman, M. M., and Hassan, M. A. 1977. A preliminary report on the angiospermic flora of Sonadia Island. Bangladesh. J. Asit. Sco. 3(1): 125-126.

Khan, M.S., Rahman, M.M. and Ali, M.A. (Eds). 2001. Red Data Book of Vascular Plants of Bangladesh. Bangladesh National Herbarium, Dhaka, pp. 1-179.

Khan, M.S. 1972-1987. Flora of Bangladesh, Fasc. 1-39. Bangladesh National Herbarium, Dhaka, Bangladesh.

Leitch, A.R. and Leitch, I.J. 2008. Genomic plasticity and the diversity of polyploid plants. Science 320(5875): 481-483.

Leuschner, W.A. and Khaleque, K. 1987. Homestead agroforestry in Bangladesh. Agroorestry Systems 5(2): 139-151.

Masum, K.M., Alam, M.S. and Abdullah-Al-Mamun, M.M., 2008. Ecological and economical significance of homestead forest to the household of the offshore island in Bangladesh. Journal of Forestry Research 19(4): 307-310.

Miah, M.G. and Hussain, M.J. 2010. Homestead agroforestry: a potential resource in Bangladesh. Sociology, Organic Farming, Climate Change and Soil Science, Springer, Dordrecht, pp. 437-463.

Muhammed, N., Masum, M.F.H., Hossain, M.M., Chakma, S., Oesten, G. and von Detten, R., 2011. Floral composition and biodiversity conservation in homestead forests in Mymensingh, Bangladesh. International Journal of Biodiversity Science, Ecosystem Services and Management 7(4): 247-257.

Muhammed, Masum, M.F.H., Hossain, M.M., Chakma, S., Oesten, G. 2013. Economic dependence of rural people on homestead forestry in Mymensingh, Bangladesh. Journal of Forestry Research 24(3): 591-597. 
Prain, D. 1903. Bengal Plants, Vols. 1 and 2, Reprint 1963. Botanical Survey of India, Calcutta.

Rahaman, M.M., Haider, M.Z. and Chakraborty, M., 2015. Contribution of Home Garden to Household Economy in Rural Areas of Bangladesh. Asia-Pacific Journal of Rural Development 25(1): 49-60.

Rahman, A.H.M.M. 2013. Angiospermic Flora of Rajshahi District, Bangladesh. American Journal of Life Sciences 1(3): 105-112.

Rahman, M. L., Hasanuzzaman, M., and Islam, M.K. 2009. Fruit distribution and diversity in the homestead of a southern island of Bangladesh. Advances in Biological Research 3(5-6): 208-214.

Rahman, M.O., Antara, R.T., Begum, M. and Hassan, M.A. 2012. Floristic diversity of Dhamrai upazila of Dhaka with emphasis on medicinal plants. Bangladesh J. Bot. 41(1): 71-85.

Rahman, S.A., Baldauf, C., Mollee, E.M., Abdullah-Al-Pavel, M., Abdullah-Al-Mamun, M., Toy, M.M. and Sunderland, T. 2013. Cultivated plants in the diversified homegardens of local communities in Ganges Valley, Bangladesh. Science Journal of Agricultural Research and Management, Article ID sjarm197, 2013: 1-6.

Roy, B., Rahman, M.H., and Fardusi, M.J. 2013. Status, diversity, and traditional uses of homestead gardens in Northern Bangladesh: A means of sustainable biodiversity conservation. Hindawi Publishing Corporation, ISRN Biodiversity, Article ID 124103, 2013: 1-11.

Sajib, N.H., Pasha, M.K., and Uddin, S.B. 2016. Angiospermic Plant Diversity of Southeast Offshore Islands in Bangladesh. Journal of Forest and Environmental Science 32(1): 27-38.

Shetu, S.S., Khan, S.A. and Uddin, S.N. 2018. Checklist of Angiosperms extant in Mirpur area of Dhaka City, Jahangirnagar University J. Biol. Sci. 7(2): 47-64.

Tabassum, R. 2015. Angiospermic flora of Gazipur district, Bangladesh. Doctoral Dissertation, Department of Botany, University of Dhaka, pp. 1-707.

The Plant List, 2013. The Plant List, a working list of all plant species. Version $1.1<\mathrm{http}: / /$ www.t heplantlist. org/>. Accessed on 23 July 2017.

TROPICOS 2010. Tropicos.org. <www.tropicos.org>. Missouri Botanical Garden, Saint Louis, Missouri, USA. Accessed on 20 November 2016.

Uddin, M.S., Rahman, M.J., Mannan, M.A., Begum, S.A., Rahman, A.F.M.F. and Haq, M.F. 2002. Plant biodiversity in the homesteads of saline area of southeastern Bangladesh. Proc. National Workshop on Agroforestry Research, Vol. 45, 54 pp.

Watson, M.F., Akiyama, S., Ikeda, H., Pendry, C.A., Rajbhandari, K.R. and Shrestha, K.K. (Eds). 2011. Flora of Nepal, Vol. 3. Magnoliaceae to Rosaceae. Royal Botanic Gardens, Edinburgh, pp.1-512.

Wu, C.Y., Raven, P.H. and Hong, D.Y. (Eds). 1995-2013. Flora of China, Vols. 2-25. Science Press and Missouri Botanical Garden Press, Beijing and St. Louis. 